\title{
RECENT DEVELOPMENTS IN CHIRAL PERTURBATION THEORY *
}

\author{
Ulf-G. Meißner ${ }^{\dagger}$ \\ Universität Bern, Institut für Theoretische Physik \\ Sidlerstr. 5, CH-3012 Bern, Switzerland
}

\begin{abstract}
I review recent developments in chiral perturbation theory (CHPT) which is the effective field theory of the standard model below the chiral symmetry breaking scale. The effective chiral Lagrangian formulated in terms of the pseudoscalar Goldstone bosons $(\pi, K, \eta)$ is briefly discussed. It is shown how one can gain insight into the ratios of the light quark masses and to what extent these statements are modelindependent. A few selected topics concerning the dynamics and interactions of the Goldstone bosons are considered. These are $\pi \pi$ and $\pi K$ scattering, some non-leptonic kaon decays and the problem of strong pionic final state interactions. CHPT also allows to make precise statements about the temperature dependence of QCD Green functions and the finite size effects related to the propagation of the (almost) massless pseudoscalar mesons. A central topic is the inclusion of matter fields, baryon CHPT. The relativistic and the heavy fermion formulation of coupling the baryons to the Goldstone fields are discussed. As applications, photo-nucleon processes, the $\pi N \Sigma$-term and non-leptonic hyperon decays are presented. Implications of the spontaneously broken chiral symmetry on the nuclear forces and meson exchange currents are also described. Finally, the use of effective field theory methods in the strongly coupled Higgs sector and in the calculation of oblique electroweak corrections is touched upon.
\end{abstract}

Commissioned article for J. Phys. G: Nucl. Part. Phys.

BUTP-93/01

January 1993

* Work supported in part by Deutsche Forschungsgemeinschaft and by Schweizerischer Nationalfonds.

$\dagger$ Heisenberg Fellow. 


\section{CONTENTS}

1. Introduction 2

2. Chiral effective Lagrangian

2.1. QCD in the presence of external sources $\quad 7$

2.2. Effective theory to lowest order 8

2.3. Chiral counting scheme 10

2.4. Effective theory at next-to-leading order 12

2.5. The low-energy constants 16

2.6. Extensions $\quad 19$

3. Light quark mass ratios

3.1. Lowest order estimates 21

3.2. Next-to-leading order estimates 22

4. The meson sector - selected topics

4.1. $\pi \pi$ scattering 29

4.2. Testing the mode of quark condensation 34

4.3. $\pi K$ scattering $\quad 36$

4.4. $\quad$ Beyond one loop 38

4.5. The decays $K \rightarrow 2 \pi$ and $K \rightarrow 3 \pi$

5. Finite temperatures and sizes

5.1. Effective theory at finite temperature 45

5.2. Melting condensates 47

5.3. Effective theory in a box $\quad 50$

5.4. Finite size effects: CHPT versus Monte Carlo 53

5.5. An application to high- $T_{c}$ superconductivity 54

6. Baryons

6.1. Relativistic formalism $\quad 57$

6.2. Non-relativistic formalism 63

6.3. Photo-nucleon processes 68

6.4. Baryon masses and the $\sigma$-term $\quad 74$

6.5. Non-leptonic hyperon decays $\quad 77$

$\begin{array}{ll}\text { 6.6. Nuclear forces and exchange currents } & 79\end{array}$

7. Strongly coupled Higgs sector

7.1. $\quad$ Basic ideas 83

7.2. $\quad$ Effective Lagrangian at next-to-leading order 86

7.3. Longitudinal vector boson scattering 88

$\begin{array}{ll}\text { 7.4. } & \text { Electroweak radiative corrections }\end{array}$

8. Miscelleaneous omissions 95

Appendix A: The case of $S U(2) \times S U(2) \quad 97$

Appendix B: $S U(3)$ meson-baryon lagrangian 98

$\begin{array}{ll}\text { References } & 99\end{array}$ 


\section{INTRODUCTION}

Effective field theories (EFTs) have become a popular tool in particle and nuclear physics. An effective field theory differs from a conventional renormalizable ("fundamental") quantum field theory in the following respect. In EFT, one only works at low energies (where "low" is defined with respect to some scale specified later) and expands the theory in powers of the energy/characteristic scale. In that case, renormalizability at all scales is not an issue and one has to handle strings of non-renormalizable interactions. Therefore, at a given order in the energy expansion, the theory is specified by a finite number of coupling (low-energy) constants (this allows e.g. for an order-by-order renormalization). All observables are parametrized in terms of these few constants and thus there is a host of predictions for many different processes. Obviously, at some high energy this effective theory fails and one has to go over to a better high energy theory (which again might be an EFT of some fundamental theory). The trace of this underlying high energy theory are the particular values of the low energy constants. The most complete and most worked with EFT is chiral perturbation theory (CHPT) which will be the central topic of this review. Before elaborating on the particular aspects of CHPT, let me make some general comments concerning the applications of EFTs.

Fig. 1: Scattering of light by light. At very low photon energies, the electrons (solid lines) can be integrated out and one is left with the EFT discussed in the text.

EFTs come into play when the underlying fundamental theory contains massless (or very light) particles. These induce poles and cuts and conventional Taylor expansions in powers of momenta fail. A typical example is QED where gauge invariance protects the photon from acquiring a mass. One photon exchange involves a propagator $\sim 1 / t$, with $t$ the invariant four-momentum transfer squared. Such a potential can not be Taylor expanded. Here, EFT comes into the game. Euler and Heisenberg [1] considered the 
scattering of light by light at very low energies, $\omega \ll m_{e}$, with $\omega$ the photon energy and $m_{e}$ the electron mass (cf. Fig.1). To calculate the scattering amplitude, one does not need full QED but rather integrates out the electron from the theory. This leads to an effective Lagrangian of the form

$$
\mathcal{L}_{\mathrm{eff}}=\frac{1}{2}\left(\vec{E}^{2}-\vec{B}^{2}\right)+\frac{e^{4}}{360 \pi^{2} m_{e}^{4}}\left[\left(\vec{E}^{2}-\vec{B}^{2}\right)^{2}+7(\vec{E} \cdot \vec{B})^{2}\right]+\ldots
$$

which is nothing but a derivative expansion since $\vec{E}$ and $\vec{B}$ contain derivatives of the gauge potential. Stated differently, since the photon energy is small, the electromagnetic fields are slowly varying. From eq.(1.1) one reads off that corrections to the leading term are suppressed by powers of $\left(\omega / m_{e}\right)^{4}$. Straightforward calculation leads to the cross section $\sigma(\omega) \sim \omega^{6} / m_{e}^{8}$. This can, of course, also be done using full QED, but the EFT caculation is much simpler. A more detailed discussion of effective field theory methods in QED can be found in the monograph by Dittrich and Reuter [2].

A similar situation arises in QCD which is a non-abelian gauge theory of colored quarks and gluons,

$$
\begin{aligned}
\mathcal{L}_{\mathrm{QCD}} & =-\frac{1}{4 g^{2}} G_{\mu \nu}^{a} G^{\mu \nu, a}+\bar{q} i \gamma^{\mu} D_{\mu} q-\bar{q} \mathcal{M} q \\
& = \\
\mathcal{L}_{\mathrm{QCD}}^{0} & +\mathcal{L}_{\mathrm{QCD}}^{\mathrm{I}}
\end{aligned}
$$

with $\mathcal{M}=\operatorname{diag}\left(m_{u}, m_{d}, m_{s}, \ldots\right)$ the quark mass matrix. For the full theory, there is a conserved charge for every quark flavor separately since the quark masses are all different. However, for the first three flavors $(u, d, s)$ it is legitimate to set the quark masses to zero since they are small on a typical hadronic scale like e.g. the $\rho$-meson mass. The absolute values of the running quark masses at $1 \mathrm{GeV}$ are $m_{u} \simeq 5 \mathrm{MeV}$, $m_{d} \simeq 9 \mathrm{MeV}, m_{s} \simeq 175 \mathrm{MeV}$, i.e. $m_{u} / M_{\rho} \simeq 0.006, m_{d} / M_{\rho} \simeq 0.012$ and $m_{s} / M_{\rho} \simeq 0.23$ [3]. If one sets the quark masses to zero, the left- and right-handed quarks defined by

$$
q_{L}=\frac{1}{2}\left(1-\gamma_{5}\right) q, \quad q_{R}=\frac{1}{2}\left(1+\gamma_{5}\right) q
$$

do not interact with each other and the whole theory admits an $U(3) \times U(3)$ symmetry. This is further reduced by the axial anomaly, so that the actual symmetry group of three flavor massless QCD is

$$
G=S U(3)_{L} \times S U(3)_{R} \times U(1)_{L+R}
$$

The U(1) symmetry related to baryon number conservation will not be discussed in any further detail. The conserved charges which come along with the chiral $S U(3) \times S U(3)$ symmetry generate the corresponding Lie algebra. In the sixties and seventies, manipulations of the commutation relations between the conserved vector $(\mathrm{L}+\mathrm{R})$ and axialvector (L-R) charges were called "PCAC relations" or "current algebra calculations" 
and lead to a host of low energy theorems and predictions [4]. These rather tedious manipulations have nowadays been replaced by EFT methods, in particular by CHPT (as will be discussed later on). Let me come back to QCD. One quickly realizes that the ground state does not have the full symmetry $G$, eq.(1.4). If that were the case, every known hadron would have a partner of the same mass but with opposite parity. Clearly, this is in contradiction with the observed particle spectrum. Another argument involves the two-point correlation functions of the vector $\left(V_{\mu}^{a}\right)$ and axial-vector $\left(A_{\mu}^{a}\right)$ currents. These can be determined from the semi-leptonic $\tau$ decays $\tau \rightarrow n \pi+\nu_{\tau}[5]$ and they show a rather different behaviour. While the $\mathrm{VV}$-correlator is peaked around $M_{\rho} \simeq 770 \mathrm{MeV}$ and smooth otherwise, the AA-correlator shows a broad enhancement around $M_{A_{1}} \simeq 1200 \mathrm{MeV}$ and is smooth elsewhere. The physical ground state must therefore be asymmetric under the chiral $S U(3)_{L} \times S U(3)_{R}[6]$. In fact, the chiral symmetry is spontaneously broken down (hidden) to the vectorial subgroup of isospin and hypercharge, generated by the vector currents,

$$
H=S U(3)_{L+R} \times U(1)_{L+R}
$$

As mandated by Goldstone's theorem [7], the spectrum of massless QCD must therefore contain $N_{f}^{2}-1=9-1=8$ massless bosons with quantum numbers $J^{P}=0^{-}$ (pseudoscalars) since the axial charges do not annihilate the vacuum. Reality is a bit more complex. The quark masses are not exactly zero which gives rise to an explicit chiral symmetry breaking (as indicated by the term $\mathcal{L}_{\mathrm{QCD}}^{I}$ in eq.(1.2)). This is in agreement with the observed particle spectrum - there are no massless strongly interacting particles. However, the eight lightest hadrons are indeed pseudoscalar mesons. These are the pions $\left(\pi^{ \pm}, \pi^{0}\right)$, the kaons $\left(K^{ \pm}, \bar{K}^{0}, K^{0}\right)$ and the eta $(\eta)$. One observes that $M_{\pi} \ll M_{K} \approx M_{\eta}$ which indicates that the masses of the quarks in the $S U(2)$ subgroup (of isospin) should be considerably smaller than the strange quark mass. This expectation is borne out by actual calculation of quark mass ratios as discussed later on. Also, from the relative size of the quark masses $m_{u, d} \ll m_{s}$ one expects the chiral expansion to converge much more rapidly in the two-flavor case than for $S U(3)_{f}$. These basic features of QCD can now be explored in a similar fashion as outlined before for the case of QED. The technical details will be given in later sections, here I just wish to outline the main ideas beyond CHPT.

As already noted, the use of EFTs in the context of strong interactions preceeds QCD. The Ward identities related to the spontaneously broken chiral symmetry were explored in great detail in the sixties in the context of current algebra and pion pole dominance [4,8]. The work of Dashen and Weinstein [9], Weinberg [10] and Callan, Coleman, Wess and Zumino [11] clarified the relation between current algebra calculations and the use of effective Lagrangians (at tree level). However, only with Weinberg's [12] seminal paper in 1979 it became clear how one could systematically generate loop corrections to the tree level (current algebra) results. In fact, he showed that these loop corrections are suppressed by powers of $(E / \Lambda)^{2}$, with $E$ a typical energy (fourmomentum) and $\Lambda$ the scale below which the EFT can be applied (typically the mass of 
the first non-Goldstone resonance, in QCD $\Lambda \simeq M_{\rho}$ ). The method was systematized by Gasser and Leutwyler for $S U(2)_{f}$ in Ref.[13] and for $S U(3)_{f}$ in Ref.[14] and has become increasingly popular ever since. The basic idea of using an effective Lagrangian instead of the full theory is based on a universality theorem for low energy properties of field theories containing massless (or very light) particles. Consider a theory (like QCD) at low energies. It exhibits the following properties:

- $\mathcal{L}$ is symmetric under some Lie group $G$ (in QCD: $\left.G=S U(3)_{L} \times S U(3)_{R}\right)$.

- The ground state $\mid 0>$ is symmetric under $H \subset G$ (in QCD: $H=S U(3)_{V}$ ). To any broken generator of $G$ there appears a massless Goldstone boson (called "pion") with the corresponding quantum numbers $\left(J^{P}=0^{-}\right.$in QCD).

- The Goldstone bosons have a finite transition amplitude to decay into the vacuum (via the current associated with the broken generators). This matrix element carries a scale $F$, which is of fundamental importance for the low energy sector of the theory (in QCD: $<0\left|A_{\mu}^{a}\right| \pi^{b}>=i p_{\mu} \delta^{a b} F$, with $F$ the pion decay constant in the chiral limit).

- There exists no other massless (strongly interacting) particles.

- Explicit symmetry breaking (like the quark mass term in QCD) can be treated in a perturbative fashion.

Now any theory with these properties looks the same (in more than two spacetime dimensions). This means that to leading order the solution to the Ward identities connected to the broken symmetry is unique and only contains the scale $F$. Thus, the EFT to lowest order is uniquely fixed and it is most economical to formulate it in terms of the Goldstone fields. In fact, one collects the pions in a matrix-valued function (generally denoted ' $U$ ') which transforms linearly under the full action of $G$. In QCD, a popular choice is $U(x)=\exp \left[i \lambda^{a} \pi^{a}(x) / F\right]$ with $\lambda^{a}(a=1, \ldots, 8)$ the Gell-Mann matrices and $U^{\prime}(x)=R U(x) L^{\dagger}$ under chiral $S U(3)_{L} \times S U(3)_{R}$ (with $L, R$ an element of $\left.S U(3)_{L, R}\right)$. Accordingly, the pion fields transform in a highly non-linear fashion $[10,11]$. This is a characteristic feature of EFTs.

While the discussion so far was mostly centered around QCD, the universality theorem immediately allows one to relate QCD to the Higgs model of standard electroweak symmetry breaking. The Higgs model has $G=O(4)$ broken down to $H=O(3)$, which is a structure isomorphic to two-flavour massless QCD. The only difference is that while in QCD one has $F \simeq 93 \mathrm{MeV}$, in the Higgs model $F \simeq 250 \mathrm{GeV}$. The longitudinal degrees of freedom of the $W^{ \pm}$and $Z^{0}$ bosons play the role of the pions as discussed in more detail later on. Where these two theories really differ is at next-to-leading order. While to leading order all Green functions are given in terms of the scale $F$ (and by some parameters related to explicit symmetry breaking), the solutions to the Ward identities at next-to-leading order can only be given modulo some unknown coefficients, the low-energy constants. These have two purposes: First, they allow to absorb the divergences inflicted by the loop contributions which have to be accounted for and, second, 
their finite values reflect the underlying high energy theory. In QCD, these low energy constants are functions of the scale $\Lambda_{\mathrm{QCD}}$ and the masses of the heavy quarks and are in principle calculable. However, at present one has to determine them phenomenologically or use some models to estimate them. This will be discussed in the following section. It is important to notice that in this way one can set up a consistent order-byorder renormalization scheme. This allows one to make predictions once one has used a certain set of processes to pin down the low-energy constants. In what follows, I will always consider EFTs beyond tree level, i.e. taking into account loop corrections. An important argument for doing this is unitarity. Tree graphs are always real and thus unitarity is violated. Evidently, loop graphs have imaginary parts and one can restore unitarity in a perturbative fashion. It was already noted long ago that there are large unitarity corrections to some processes [15], but it should be stressed that there are also other corrections. CHPT accounts for all of them in a systematic manner. The general framework of EFTs is therefore based on some general principles like Lorentz invariance, analyticity, unitarity and cluster decomposition. If one has a finite number of massless or light particle types, one simply writes down an effective non-linear Lagrangian of these particles in accordance with the pertinent symmetry requirements. Corrections to the leading order predictions can be worked out in a systematic fashion. For doing that, one invokes higher dimensional operators whose contributions are suppressed by powers of $1 / M_{\text {new }}$, with $M_{\text {new }}$ a typical scale of new physics (like the $\rho$ in QCD or the techni-rho in technicolor). As we will see in what follows, there exists special cases were the one loop corrections are already large at low energies. This forces one to go beyond the generally well working one loop approximation (as will be discussed in some detail later on).

We are now in the position to consider more specific examples build on these general ideas. The material is organized as follows. In section II, I review the basic construction of the next-to-leading order chiral Lagrangian of the strong interactions. It also contains the pertinent chiral counting rules which are at the heart of the systematic expansion in small momenta and quark masses. Extensions to incorporate the energy-momentum tensor and the $U(1)_{A}$ anomaly are briefly touched upon. The physics related to the anomalous sector (Wess-Zumino-Witten term) is not discussed in any detail. In section III, it is shown how chiral perturbation theory can be used to gain insight about the ratios of the light quark masses. Section IV is concerned with some applications in the meson sector like the classical field of pion-pion scattering. I also elucidate some problems due to strong pionic final state interactions. The extension to non-leptonic weak decays is briefly discussed in the context of the decays $K \rightarrow 2 \pi, 3 \pi$. In section $\mathrm{V}$ effects of finite temperatures and volumes are considered. In particular, the melting of the quark and gluon condensates and finite size effects in relation to lattice gauge theories are discussed. The following section VI is devoted to the field of baryon CHPT, with special emphasis on the newly developed methods from heavy quark EFTs and applications to photo-nucleon processes. In section VII, it is shown how CHPT methods can be used in the strongly interacting Higgs sector. The topics of $W_{L} W_{L}$ scattering and 
oblique corrections to the $Z$ propagator are discussed. In section VIII, miscellaneous applications and developments are touched upon.

\section{CHIRAL EFFECTIVE LAGRANGIAN}

In this section, I briefly review how to construct the effective chiral Lagrangian of the strong interactions at next-to-leading order, following closely the work of Gasser and Leutwyler [14]. It is most economical to use the external field technique since it avoids any complication related to the non-linear transformation properties of the pions. The basic objects to consider are currents and densities with external fields coupled to them [16] in accordance with the symmetry requirements. The associated Green functions automatically obey the pertinent Ward identities and higher derivative terms can be constructed systematically. The S-matrix elements for processes involving physical mesons follow then via standard LSZ reduction.

\subsection{QCD in the presence of external sources}

Consider the vacuum-to-vacuum transition amplitude in the presence of external fields

$$
\mathrm{e}^{i \mathcal{Z}[v, a, s, p]}=<0 \text { out } \mid 0 \text { in }>_{v, a, s, p}
$$

based on the QCD Lagrangian

$$
\begin{aligned}
& \mathcal{L}=\mathcal{L}_{\mathrm{QCD}}^{0}+\bar{q}\left(\gamma^{\mu} v_{\mu}(x)+\gamma^{5} \gamma^{\mu} a_{\mu}(x)\right) q-\bar{q}(s(x)-i p(x)) q-\frac{\theta(x)}{16 \pi^{2}} G_{\mu \nu}^{a} \tilde{G}^{\mu \nu, a} \\
& \mathcal{L}_{\mathrm{QCD}}^{0}=-\frac{1}{2 g^{2}} G_{\mu \nu}^{a} G^{\mu \nu, a}+\bar{q} i \gamma^{\mu}\left(\partial_{\mu}-i A_{\mu}\right) q
\end{aligned}
$$

with $A_{\mu}$ the gluon field, $G_{\mu \nu}$ the corresponding field strength tensor and $\tilde{G}_{\mu \nu}=$ $\frac{1}{2} \epsilon_{\mu \nu \alpha \beta} G^{\alpha \beta}$ its dual. $\mathcal{L}_{\mathrm{QCD}}^{0}$ is massless $\mathrm{QCD}$ with vanishing vacuum angle. The external vector $\left(v_{\mu}\right)$, axial-vector $\left(a_{\mu}\right)$, pseudoscalar $(p)$ and scalar $(s)$ fields are hermitean $3 \times 3$ matrices in flavor space. The quark mass matrix $\mathcal{M}$,

$$
\mathcal{M}=\operatorname{diag}\left(m_{u}, m_{d}, m_{s}\right)
$$

is contained in the scalar field $s(x)$. The Green functions of massless QCD are obtained by expanding the generating functional around $v_{\mu}=a_{\mu}=s=p=0$ and $\theta(x)=\theta_{0}$. For the real world, one has to expand around $v_{\mu}=a_{\mu}=p=0, s(x)=\mathcal{M}$ and $\theta(x)=\theta_{0}$. The Lagrangian $\mathcal{L}$ is invariant under local $S U(3) \times S U(3)$ chiral transformations if the quark and external fields transform as follows:

$$
\begin{aligned}
q_{R}^{\prime} & =R q \quad ; \quad q_{L}^{\prime}=L q \\
v_{\mu}^{\prime}+a_{\mu}^{\prime} & =R\left(v_{\mu}+a_{\mu}\right) R^{\dagger}+i R \partial_{\mu} R^{\dagger} \\
v_{\mu}^{\prime}-a_{\mu}^{\prime} & =L\left(v_{\mu}-a_{\mu}\right) L^{\dagger}+i L \partial_{\mu} L^{\dagger} \\
s^{\prime}+i p^{\prime} & =R(s+i p) L^{\dagger}
\end{aligned}
$$


with $L, R$ elements of $S U(3)_{L, R}$ (in general, these are elements of $U(3)_{L, R}$, but we already account for the axial anomaly to be discussed later). The path integral representation of $\mathcal{Z}$ reads:

$$
\mathrm{e}^{i \mathcal{Z}[v, a, s, p]}=\int\left[D A_{\mu}\right][D q][D \bar{q}] \mathrm{e} \int^{\int i d^{4} x \mathcal{L}\left(q, \bar{q}, G_{\mu \nu} ; v, a, s, p\right)}
$$

It allows one to make contact to the effective meson theory. Since we are interested in processes were the momenta are small (the low energy sector of the theory), we can expand the Green functions in powers of the external momenta. This amounts to an expansion in derivatives of the external fields. As already pointed out, the low energy expansion is not a simple Taylor expansion since the Goldstone bosons generate poles at $q^{2}=0$ (in the chiral limit) or $q^{2}=M_{\pi}^{2}$ (for finite quark masses). The low energy expansion involves two small parameters, the external momenta $q$ and the quark masses $\mathcal{M}$. One expands in powers of these with the ratio $\mathcal{M} / q^{2}$ fixed. The effective meson Lagrangian to carry out this procedure follows from the low energy representation of the generating functional

$$
\mathrm{e}^{i \mathcal{Z}[v, a, s, p]}=\int[D U] \mathrm{e} \int^{\int i d^{4} x \mathcal{L}_{\text {eff }}(U ; v, a, s, p)}
$$

where the matrix $U$ collects the meson fields. The low energy expansion is now obtained from a perturbative expansion of the meson EFT,

$$
\mathcal{L}_{\text {eff }}=\mathcal{L}_{2}+\mathcal{L}_{4}+\ldots
$$

where the subscript $(n=2,4, \ldots)$ denotes the low energy dimension (number of derivatives and/or quark mass terms). The various terms in this expansion will now be discussed.

\subsection{Effective theory to leading order}

Let us now construct the leading term (called $\mathcal{L}_{2}$ ) in the low energy expansion $(2.7)$. The mesons are described by a unitary $3 \times 3$ matrix in flavor space,

$$
U^{\dagger} U=1, \quad \operatorname{det} U=1
$$

The matrix $U$ transforms linearly under chiral symmetry, $U^{\prime}=R U L^{\dagger}$. The lowest order Lagrangian consistent with Lorentz invariance, chiral symmetry, parity, G-parity and charge conjugation reads [14]

$$
\mathcal{L}=\frac{1}{4} F^{2}\left\{\operatorname{Tr}\left[\nabla_{\mu} U^{\dagger} \nabla^{\mu} U+\chi^{\dagger} U+\chi U^{\dagger}\right]\right\}+\frac{1}{12} H_{0} \nabla_{\mu} \theta \nabla^{\mu} \theta
$$


The covariant derivative $\nabla_{\mu} U$ transforms linearly under chiral $S U(3) \times S U(3)$ and contains the couplings to the external vector and axial fields,

$$
\nabla_{\mu} U=\partial_{\mu} U-i\left(v_{\mu}+a_{\mu}\right) U+i U\left(v_{\mu}-a_{\mu}\right)
$$

The field $\chi$ embodies the scalar and pseudoscalar externals,

$$
\chi=2 B(s+i p)
$$

The last term in (2.9) is related to $\mathrm{CP}$ violation in the strong interaction. For the moment, we will set this term to zero (which agrees with the empirical observation that the electric dipole moment of the neutron is tiny). Let us now discuss the various constants appearing in eqs. $(2.9,2.11)$. The scale $F$ is related to the axial vector currents,

$$
A_{\mu}^{a}=-F \partial_{\mu} \pi^{a}+\ldots
$$

and thus can be identified with the pion decay constant in the chiral limit, $F=F_{\pi}\{1+$ $\mathcal{O}(\mathcal{M})\}$, by direct comparison with the matrix-element $<0\left|A_{\mu}^{a}\right| \pi^{b}>=i p_{\mu} \delta^{a b} F$. The constant $B$, which appears in the field $\chi$, is related to the explicit chiral symmetry breaking. Consider the symmetry breaking part of the Lagrangian and expand it in powers of the pion fields (with $p=0, s=\mathcal{M}$ so that $\chi=2 B \mathcal{M}$ )*

$$
\mathcal{L}_{2}^{S B}=\frac{1}{2} F^{2} B \operatorname{Tr}\left[\mathcal{M}\left(U+U^{\dagger}\right)\right]=\left(m_{u}+m_{d}+m_{s}\right) B\left[F^{2}-\frac{\pi^{2}}{2}+\frac{\pi^{4}}{24 F^{2}}+\ldots\right]
$$

with $\pi=\lambda^{a} \pi^{a}$. The first term on the right hand side of eq.(2.13) is obviously related to the vacuum energy, while the second and third are meson mass and interaction terms, respectively. Since $\partial H_{\mathrm{QCD}} / \partial m_{q}=\bar{q} q$ it follows from (2.13) that

$$
<0|\bar{u} u| 0>=<0|\bar{d} d| 0>=<0|\bar{s} s| 0>=-F^{2} B\{1+\mathcal{O}(\mathcal{M})\}
$$

This shows that the constant $B$ is related to the vev's of the scalar quark densities $<0|\bar{q} q| 0>$, the order parameter of the spontaneous chiral symmetry breaking. The relation (2.14) is only correct modulo higher order corrections in the quark masses as indicated by the term $\mathcal{O}(\mathcal{M})$. One can furthermore read off the pseudoscalar mass terms from $(2.13)$. In the case of isospin symmetry $\left(m_{u}=m_{d}=\hat{m}\right)$, one finds

$$
\begin{aligned}
M_{\pi}^{2} & =2 \hat{m} B\{1+\mathcal{O}(\mathcal{M})\}=\stackrel{\circ}{M}_{\pi}^{2}\{1+\mathcal{O}(\mathcal{M})\} \\
M_{K}^{2} & =\left(\hat{m}+m_{s}\right) B\{1+\mathcal{O}(\mathcal{M})\}=\stackrel{\circ}{M}_{K}^{2}\{1+\mathcal{O}(\mathcal{M})\} \\
M_{\eta}^{2} & =\frac{2}{3}\left(\hat{m}+2 m_{s}\right) B\{1+\mathcal{O}(\mathcal{M})\}=\stackrel{\circ}{M}_{\eta}^{2}\{1+\mathcal{O}(\mathcal{M})\}
\end{aligned}
$$

\footnotetext{
* remember that $\pi$ stands as a generic symbol for the pions, kaons and the $\eta$.
} 
with $\stackrel{\circ}{M}_{P}$ denoting the leading term in the quark mass expansion of the pseudoscalar

meson masses. For the $\stackrel{\circ}{M}_{P}$, the Gell-Mann-Okubo relation is exact, $4 \stackrel{\circ}{M}_{K}^{2}=\stackrel{\circ}{M}_{\pi}^{2}+3 \stackrel{\circ}{M}_{\eta}^{2}$ [17]. In the case of isospin breaking, which leads to $\pi^{0}-\eta$ mixing, these mass formulae are somewhat more complicated (see section 3 and ref.[14]). Eq.(1.15) exhibits nicely the Goldstone character of the pions - when the quark masses are set to zero, the pseudoscalars are massless and $S U(3) \times S U(3)$ is an exact symmetry. For small symmetry breaking, the mass of the pions is proportional to the square root of the symmetry breaking parameter, i.e. the quark masses (an alternative scenario is discussed in section 4.2). From eqs.(2.13) and (2.15) one can eliminate the constant $B$ and gets the celebrated Gell-Mann-Oakes-Renner [18] relations

$$
\begin{aligned}
F_{\pi}^{2} M_{\pi}^{2} & =-2 \hat{m}<0|\bar{u} u| 0>+\mathcal{O}\left(\mathcal{M}^{2}\right) \\
F_{K}^{2} M_{K}^{2} & =-\left(\hat{m}+m_{s}\right)<0|\bar{u} u| 0>+\mathcal{O}\left(\mathcal{M}^{2}\right) \\
F_{\eta}^{2} M_{\eta}^{2} & =-\frac{2}{3}\left(\hat{m}+m_{s}\right)<0|\bar{u} u| 0>+\mathcal{O}\left(\mathcal{M}^{2}\right)
\end{aligned}
$$

where we have used $F_{P}=F\{1+\mathcal{O}(\mathcal{M})\}(P=\pi, K, \eta)$, i.e. the differences in the physical decay constants $F_{\pi} \neq F_{K} \neq F_{\eta}$ appear in the terms of order $\mathcal{M}^{2}$.

From this discussion we realize that to leading order the strong interactions are characterized by two scales, namely $F$ and $B$. Numerically, using the sum rule value $<0|\bar{u} u| 0>=(-225 \mathrm{MeV})^{3}[19]$, one has

$$
\begin{aligned}
& F \simeq F_{\pi} \simeq 93 \mathrm{MeV} \\
& B \simeq 1300 \mathrm{MeV}
\end{aligned}
$$

The large value of the ratio $B / F \simeq 14$ has triggered some investigations of alternative scenarios concerning the mode of quark condensation as will be discussed later.

One can now calculate tree diagrams using the effective Lagrangian $\mathcal{L}_{2}$ and derive with ease all so-called current algebra predictions (low energy theorems). Current algebra is, as should have become evident by now, only the first term in a systematic low energy expansion. Working out tree graphs using $\mathcal{L}_{2}$ can not be the whole story tree diagrams are always real and thus unitarity is violated. One has to include higher order corrections to cure this. To do this in a consistent fashion, one needs a counting scheme to be discussed next.

\subsection{Chiral counting scheme}

The leading term in the low energy expansion of $\mathcal{L}_{\text {eff }}(2.7)$ was denoted $\mathcal{L}_{2}$ because it has dimension (chiral power) two. It contains two derivatives or one power of the quark mass matrix. If one assumes the matrix $U$ to be order one, $U=\mathcal{O}(1)$, a consistent power counting scheme for local terms containing $U, \partial_{\mu} U, v_{\mu}, a_{\mu}, s, p, \ldots$ goes as follows. Denote by $q$ a generic small momentum (for an exact definition of 'small', 
see eq.(2.27)). Derivatives count as order $q$ and so do the external fields which occur linearly in the covariant derivative $\nabla_{\mu} U$. For the scalar and pseudoscalar fields, it is most convenient to book them as order $q^{2}$. This can be traced back to the fact that the scalar field $s(x)$ contains the quark mass matrix, thus $s(x) \sim \mathcal{M} \sim M_{\pi}^{2} \sim q^{2}$. With these rules, all terms appearing in (2.9) are of order $q^{2}$, thus the notation $\mathcal{L}_{2}$ (notice that a term of order one is a constant since $U^{\dagger} U=1$ and can therefore be disregarded. Odd powers of $q$ clash with parity requirements). To summarize, the building blocks of all terms containing derivatives and/or quark masses have the following dimension:

$$
\begin{aligned}
& \partial_{\mu} U(x), v_{\mu}(x), a_{\mu}(x)=\mathcal{O}(q) \\
& s(x), p(x), F_{\mu \nu}^{L, R}(x)=\mathcal{O}\left(q^{2}\right)
\end{aligned}
$$

where we have introduced the field strength $F_{\mu \nu}^{L, R}$ for later use. They are defined via

$$
\begin{aligned}
& F_{\mu \nu}^{I}=\partial_{\mu} F_{\nu}^{I}-\partial_{\nu} F_{\mu}^{I}-i\left[F_{\mu}^{I}, F_{\nu}^{I}\right], \quad I=L, R \\
& F_{\mu \nu}^{R}=v_{\mu}+a_{\mu} ; \quad F_{\mu \nu}^{L}=v_{\mu}-a_{\mu}
\end{aligned}
$$

As already mentioned, unitarity calls for pion loop graphs. Weinberg [12] made the important observation that diagrams with $n(n=1,2, \ldots)$ meson loops are suppressed by powers of $\left(q^{2}\right)^{n}$ with respect to the leading term. His rather elegant argument goes as follows. Consider the S-matrix for a reaction involving $N_{e}$ external pions

$$
S=\delta\left(p_{1}+p_{2}+\ldots+p_{N_{e}}\right) M
$$

with $M$ the transition amplitude. The dimension of $M$ is $[M]=4-N_{e}$ since any external pion wave function scales like $[\text { mass }]^{-1}$ according to the usual PCAC relation. Now $M$ depends on the total momentum flowing through the amplitude, on the pertinent coupling constants $g$ and the renormalization scale $\mu$ (the loop diagrams are in general divergent and need to be regularized),

$$
\begin{aligned}
M & =M(q, g, \mu)=q^{D} f(q / \mu, g) \\
D & =4-N_{e}-\Delta
\end{aligned}
$$

Here, $\Delta$ is the dimension of the different couplings and propagators entering the transition amplitude. For the effective meson theories under consideration, the coupling constants associated with the pionic interactions scale as $4-d$, with $d$ the number of derivatives as mandated by Goldstone's theorem (in the chiral limit). If $M$ contains $N_{d}$ such vertices and $N_{i}$ internal propagators, we have

$$
\Delta=\sum_{d} N_{d}(d-4)-2 N_{i}-N_{e}
$$


The total number of loops $\left(N_{l}\right)$ follows from simple topological arguments to be

$$
N_{e}=N_{i}-\sum_{d} N_{d}-1
$$

so that the total scaling dimension $D$ of $M$ is

$$
D=2+\sum_{d} N_{d}(d-2)+2 N_{l}
$$

The dominant graphs at low energy carry the smallest value of $D$. The leading terms with $d=2$ scale like $q^{2}$ at tree level $\left(N_{l}=0\right)$, like $q^{4}$ at one loop level $\left(N_{l}=1\right)$ and so on. Higher derivative terms with $d=4$ scale as $q^{4}$ at tree level, as $q^{6}$ at one-loop order etc. This power suppression of loop diagrams is at the heart of the low energy expansion in EFTs like e.g. CHPT.

Up to now, I have been rather casual with the meaning of the word "small". By small momentum or small quark mass I mean this with respect to some typical hadronic scale, also called the scale of chiral symmetry breaking (denoted by $\Lambda_{\chi}$ ). Georgi and Manohar [20] have argued that a consistent chiral expansion is possible if

$$
\Lambda_{\chi} \leq 4 \pi F_{\pi} \simeq 1 \mathrm{GeV}
$$

Their argument is based on the observation that under a change of the renormalization scale of order one typical loop contributions (say to the $\pi \pi$ scattering amplitude) will correspond to changes in the effective couplings of the order $F_{\pi}^{2} / \Lambda_{\chi}^{2} \simeq 1 /(4 \pi)^{2}$. Setting $\Lambda_{\chi}=4 \pi F_{\pi}$ and cutting the logarithmically divergent loop integrals at this scale, quantum corrections are of the same order of magnitude as changes in the renormalized interaction terms. The factor $(4 \pi)^{2}$ is related to the dimensionality of the loop integrals (for a more detailed outline of this argument see the monograph by Georgi [21]). Another type of argument was already mentioned in section 1 . Consider $\pi \pi$ scattering in the $I=J=1$ channel. There, at $\sqrt{s}=770 \mathrm{MeV}$, one hits the $\rho$-resonance. This is a natural barrier to the derivative expansion of the Goldstone mesons and therefore serves as a cut off. The appearance of the $\rho$ signals the regime of the non-Goldstone particles and describes new physics. It is therefore appropriate to choose

$$
\Lambda_{\chi} \simeq M_{\rho} \simeq 770 \mathrm{MeV}
$$

which is not terribly different from the estimate (2.25). In summary, small external momenta $q$ and small quark masses $\mathcal{M}$ means

$$
q / M_{\rho} \ll 1 ; \mathcal{M} / M_{\rho} \ll 1 .
$$

\subsection{Effective theory at next-to-leading order}


We have now assembled all tools to discuss the generating functional $\mathcal{Z}$ at nextto-leading order, i.e. at $\mathcal{O}\left(q^{4}\right)$. It consists of three different contributions:

1) The anomaly functional is of order $q^{4}$ (it contains four derivatives). We denote the corresponding functional by $\mathcal{Z}_{A}$. The explicit construction was given by Wess and Zumino [22] and can also be found in ref.[14]. A geometric interpretation is provided by Witten [23].

2) The most general effective Lagrangian of order $q^{4}$ which is gauge invariant. It leads to the action $\mathcal{Z}_{2}+\mathcal{Z}_{4}=\int d^{4} x \mathcal{L}_{2}+\int d^{4} x \mathcal{L}_{4}$.

3) One loop graphs associated with the lowest order term, $\mathcal{L}_{2}$. These also scale as terms of order $q^{4}$.

Let me first discuss the anomaly functional $\mathcal{Z}_{A}$. It subsumes all interactions which break the intrinsic parity and is responsible e.g. for the decay $\pi^{0} \rightarrow 2 \gamma$. It also generates interactions between five or more Goldstone bosons [23]. In what follows, we will not consider this sector in any detail (for a review, see ref.[24] and recent work in the context of CHPT is quoted in section 8).

What is now the most general Lagrangian at order $q^{4}$ ? The building blocks and their low energy dimensions were already discussed - we can have terms with four derivatives and one quark mass or with two quark masses (and, correspondingly, the other external fields). In $S U(3)$, the only invariant tensors are $g_{\mu \nu}$ and $\epsilon_{\mu \nu \alpha \beta}$, so one is left with (imposing also P, G and gauge invariance)

$$
\mathcal{L}=\sum_{i=1}^{10} L_{i} P_{i}+\sum_{j=1}^{2} H_{j} \tilde{P}_{j}
$$

with

$$
\begin{aligned}
P_{1} & =\operatorname{Tr}\left(\nabla^{\mu} U^{\dagger} \nabla_{\mu} U\right)^{2} \\
P_{2} & =\operatorname{Tr}\left(\nabla_{\mu} U^{\dagger} \nabla_{\nu} U\right) \operatorname{Tr}\left(\nabla^{\mu} U^{\dagger} \nabla^{\nu} U\right) \\
P_{3} & =\operatorname{Tr}\left(\nabla^{\mu} U^{\dagger} \nabla_{\mu} U \nabla^{\nu} U^{\dagger} \nabla_{\nu} U\right) \\
P_{4} & =\operatorname{Tr}\left(\nabla^{\mu} U^{\dagger} \nabla_{\mu} U\right) \operatorname{Tr}\left(\chi^{\dagger} U+\chi U^{\dagger}\right) \\
P_{5} & =\operatorname{Tr}\left(\nabla^{\mu} U^{\dagger} \nabla_{\mu} U\right)\left(\chi^{\dagger} U^{\prime} \chi U^{\dagger}\right) \\
P_{6} & =\left[\operatorname{Tr}\left(\chi^{\dagger} U+\chi U^{\dagger}\right)\right]^{2} \\
P_{7} & =\left[\operatorname{Tr}\left(\chi^{\dagger} U-\chi U^{\dagger}\right)\right]^{2} \\
P_{8} & =\operatorname{Tr}\left(\chi^{\dagger} U \chi^{\dagger} U+\chi U^{\dagger} \chi U^{\dagger}\right) \\
P_{9} & =-i \operatorname{Tr}\left(F_{\mu \nu}^{R} \nabla^{\mu} U \nabla^{\nu} U^{\dagger}\right) \operatorname{Tr}\left(F_{\mu \nu}^{L} \nabla^{\mu} U^{\dagger} \nabla^{\nu} U\right) \\
P_{10} & =\operatorname{Tr}\left(U^{\dagger} F_{\mu \nu}^{R} U F^{L, \mu \nu}\right) \\
\tilde{P}_{1} & =\operatorname{Tr}\left(F_{\mu \nu}^{R} U F^{R, \mu \nu}+F_{\mu \nu}^{L} U F^{L, \mu \nu}\right) \\
\tilde{P}_{2} & =\operatorname{Tr}\left(\chi^{\dagger} \chi\right)
\end{aligned}
$$


For the two flavor case, not all of these terms are independent. The pertinent $q^{4}$ effective Lagrangian is discussed in Appendix A. The first ten terms of (2.28) are of physical relevance for the low energy sector, the last two are only necessary for the consistent renormalization procedure discussed below. These terms proportional to $\tilde{P}_{j}(j=1,2)$ do not contain the Goldstone fields and are therefore not directly measurable at low energies. The constants $L_{i}(i=1, \ldots, 10)$ appearing in $(2.28)$ are the so-called lowenergy constants. They are not fixed by the symmetry and have the generic structure

$$
L_{i}=L_{i}^{r}+L_{i}^{\mathrm{inf}}
$$

These constants serve to renormalize the infinities of the pion loops $\left(L_{i}^{\mathrm{inf}}\right)$ and the remaining finite pieces $\left(L_{i}^{r}\right)$ have to be fixed phenomenologically or to be estimated by some model (see below). At next-to-leading order, the strong interactions dynamics is therefore determined in terms of twelve parameters $-B, F, L_{1}, \ldots, L_{10}$ (remember that we have disregarded the singlet vector and axial currents). In the absence of external fields, only the first three terms in (2.28) have to be retained.

Finally, we have to consider the loops generated by the lowest order effective Lagrangian. These are of dimension $q^{4}$ (one loop approximation) as mandated by Weinberg's scaling rule. To evaluate these loop graphs one considers the neighbourhood of the solution $\bar{U}(x)$ to the classical equations of motion. In terms of the generating functional, this reads

$$
\mathrm{e}^{i \mathcal{Z}}=\mathrm{e}^{i \int d^{4} x\left[\mathcal{L}_{2}(\bar{U})+\mathcal{L}_{4}(\bar{U})\right]} \int[D U] \mathrm{e}^{i \int d^{4} x\left[\mathcal{L}_{2}(U)-\mathcal{L}_{2}(\bar{U})\right]}
$$

The bar indicates that the Lagrangian is evaluated at the classical solution. According to the chiral counting, in the second factor of (2.31) only the term $\mathcal{L}_{2}$ is kept. This leads to

$$
\mathcal{Z}=\int d^{4} x\left(\overline{\mathcal{L}}_{2}+\overline{\mathcal{L}}_{4}\right)+\frac{i}{2} \ln \operatorname{det} D
$$

The operator $D$ is singular at short distances. The ultraviolet divergences contained in $\ln \operatorname{det} D$ can be determined via the heat kernel expansion [25]. Using dimensional regularization, the UV divergences in four dimensions take the form

$$
-\frac{1}{(4 \pi)^{2}} \frac{1}{d-4} \operatorname{Sp}\left(\frac{1}{2} \hat{\sigma}^{2}+\frac{1}{12} \hat{\Gamma}_{\mu \nu} \hat{\Gamma}^{\mu \nu}\right)
$$

The explicit form of the operators $\hat{\sigma}$ and $\hat{\Gamma}_{\mu \nu}$ can be found in ref.[14]. Using their explicit expressions, the poles in $\ln \operatorname{det} D$ can be absorbed by the following renormalization of the low energy constants:

$$
\begin{aligned}
L_{i} & =L_{i}^{r}+\Gamma_{i} \lambda, \quad i=1, \ldots, 10 \\
H_{j} & =H_{j}^{r}+\tilde{\Gamma}_{j} \lambda, \quad j=1,2
\end{aligned}
$$


with

$$
\begin{aligned}
\lambda & =\frac{1}{16 \pi^{2}} \mu^{d-4}\left\{\frac{1}{d-4}-\frac{1}{2}\left[\ln (4 \pi)+\Gamma^{\prime}(1)+1\right]\right\} \\
\Gamma_{1} & =\frac{3}{32}, \quad \Gamma_{2}=\frac{3}{16}, \quad \Gamma_{3}=0, \quad \Gamma_{4}=\frac{1}{8} \\
\Gamma_{5} & =\frac{3}{8}, \quad \Gamma_{6}=\frac{11}{144}, \quad \Gamma_{7}=0, \quad \Gamma_{8}=\frac{5}{48} \\
\Gamma_{9} & =\frac{1}{4}, \quad \Gamma_{10}=-\frac{1}{4}, \quad \tilde{\Gamma}_{1}=-\frac{1}{8}, \quad \tilde{\Gamma}_{2}=\frac{5}{24}
\end{aligned}
$$

and $\mu$ is the scale of dimensional regularization. The $q^{4}$ contribution $\mathcal{Z}_{4}+\mathcal{Z}_{1-\text { loop }}$ is finite at $d=4$ when expressed in terms of the renormalized coupling constants $L_{i}^{r}$ and $H_{i}^{r}$. The next step consists in the expansion of the differential operator $D$ in powers of the external fields. This gives the explicit contributions of the one-loop graphs to a given Green function. The full machinery is spelled out in Gasser and Leutwyler [14]. In general, one groups the loop contributions into tadpole and unitarity corrections. While the tadpoles contain one vertex and one loop, the unitarity corrections contain one loop and two vertices. The tadpole contributions renormalize the couplings of the effective Lagrangian. Both of these loop contributions also depend on the scale of dimensional regularization. In contrast, physical observables are $\mu$-independent. For actual calculations, however, it is sometimes convenient to choose a particular value of $\mu$, say, $\mu=M_{\eta}$ or $\mu=M_{\rho}$.

Fig. 2: Goldstone boson scattering in the one-loop approximation. At lowest order only tree diagrams (a) contribute. At next-to-leading order, one has tadpole (b), unitarity (c) and higher derivative (as denoted by the black box in (d)) corrections. 
To one-loop order, the generating functional therefore takes the form

$$
\mathcal{Z}=\mathcal{Z}_{2}+\mathcal{Z}_{4}+\mathcal{Z}_{\text {one-loop }}+\mathcal{Z}_{\text {anom }}
$$

and what remains to be done is to determine the values of the renormalized low energy constants, $L_{i}^{r}(i=1, \ldots, 10)$. Before doing this, let me graphically show the one-loop approximation to the $\pi \pi$-scattering amplitude (fig.2). The first term on the right hand side is the famous tree level term which leads to Weinberg's current algebra prediction for the scattering amplitude. The next two terms depict a tadpole and a unitarity correction. Finally, the term with the black box is a higher derivative term accompanied by a low energy constant. The analytical form of this amplitude will be discussed in section 4 .

\subsection{The low-energy constants}

The low energy constants are in principle calculable from QCD, they depend on $\Lambda_{\mathrm{QCD}}$ and the heavy quark masses

$$
L_{i}^{r}=L_{i}^{r}\left(\Lambda_{\mathrm{QCD}} ; m_{c}, m_{b}, m_{t}\right)
$$

In practice, such a calculation is not feasible. One therefore resorts to phenomenology and determines the $L_{i}^{r}$ from data. However, some of these constants are not easily extracted from empirical information. Therefore, one uses constraints from the large $N_{c}$ world $[16,27]$. In the limit of $N_{c}$ (with $N_{c}$ the number of colors) going to infinity and keeping $g^{2} N_{c}$ fixed, the Green functions are proportional to some power of $N_{c}[26,27$, 28]. Furthermore, in this limit the $\eta^{\prime}$ becomes massless, $M_{\eta^{\prime}} \sim 1 / N_{c}$. This leads to an enhancement of the coupling $L_{7}$,

$$
L_{7}^{\eta^{\prime}}=-\frac{\gamma^{2}}{48} \frac{F^{2}}{M_{\eta}^{2}}
$$

with $\gamma$ measuring the strength of $\eta \eta^{\prime}$ mixing. The large $-N_{c}$ counting rules for all $L_{i}^{r}$ have been worked out by Gasser and Leutwyler [14]:

$$
\begin{aligned}
\mathcal{O}\left(N_{c}^{2}\right): & L_{7} \\
\mathcal{O}\left(N_{c}\right): & L_{1}, L_{2}, L_{3}, L_{4}, L_{8}, L_{10} \\
\mathcal{O}(1): & 2 L_{1}-L_{2}, L_{4}, L_{6}
\end{aligned}
$$

Using this and experimental information from $\pi \pi$ scattering, $F_{K} / F_{\pi}$, the electromagnetic radius of the pion and so on, one ends up with the values for the $L_{i}^{r}\left(\mu=M_{\eta}\right)$ given in table 1 (large $N_{c}$ arguments are used to estimate $L_{1}, L_{4}$ and $L_{6}$ ). For comparison, we also give the values at $\mu=M_{\rho}$. It should be noted that the large $N_{c}$ suppression of $2 L_{1}-L_{2}$ has recently been tested using data from $K_{\ell 4}$ decay. Riggenbach et al. 
[29] find $\left(2 L_{1}-L_{2}\right) / L_{3}=-0.19_{-0.80}^{+0.55}$, i.e. the large $N_{c}$ prediction works within one standard deviation. More accurate data will allow to further pin down this quantity. In the case of $S U(2)$, one can define scale-independent couplings $\bar{\ell}_{i}(i=1, \ldots, 7)$. These are discussed in appendix A.

Can one now understand the values of the $L_{i}^{r}$ from some first principles? Already in their 1984 paper, Gasser and Leutwyler [13] made the following observation. They considered an effective theory of $\rho$ mesons coupled to the pseudoscalars. Eliminating the heavy field by use of the equations of motion in the region of momenta much smaller than the $\rho$ mass, one ends up with terms of order $q^{4}$. The values of the corresponding low energy constants are given in terms of $M_{\rho}$ and the $\rho$-meson coupling strengths to photons and pions. This leads to a fair description of the low energy constants. To make this statement more transparent, consider the vector form factor of the pion

$$
<\pi^{+}\left(p^{\prime}\right)\left|J_{\mu}^{\mathrm{em}}\right| \pi^{+}(p)>=F_{V}(t)\left(p+p^{\prime}\right)_{\mu}
$$

with $t=\left(p^{\prime}-p\right)^{2}$. For small $t$, the form factor can be Taylor expanded,

$$
F_{V}(t)=1+\frac{1}{6}<r^{2}>_{V}^{\pi} t+\mathcal{O}\left(t^{2}\right)
$$

Calculating $F_{V}(t)$ with $\mathcal{L}_{2}$ alone, one gets $F_{V}(t)=1$. The one-loop result has the form

$$
\begin{aligned}
F_{V}(t)=1 & +\left[\frac{2 L_{9}}{F_{\pi}^{2}}-\frac{1}{96 \pi^{2} F_{\pi}^{2}}\left(\ln \left(\frac{M_{\pi}^{2}}{\mu^{2}}\right)-\frac{1}{3}\right)\right] t \\
& +\frac{t-4 M_{\pi}^{2}}{96 \pi^{2} F_{\pi}^{2}}\left[2+\sigma \ln \left|\frac{\sigma-1}{\sigma+1}\right|+i \pi \Theta\left(t-4 M_{\pi}^{2}\right)\right]
\end{aligned}
$$

with $\sigma=\sqrt{1-4 M_{\pi}^{2} / t}$. The last term contains the imaginary part required by unitarity and some rescattering corrections. Clearly, one has to pin down $L_{9}^{r}$ to have a prediction for the form factor. As it is well known, $F_{V}(t)$ is well described within the vector meson dominance (VMD) picture,

$$
F_{V}(t)=\frac{M_{\rho}^{2}}{M_{\rho}^{2}-t}=1+\frac{t}{M_{\rho}^{2}}+\frac{t^{2}}{M_{\rho}^{4}}+\ldots
$$

where I have neglected imaginary parts and alike (a better form is given in Ref.[30]). Of course, the expansion in $t / M_{\rho}^{2}$ is only useful as long as $t / M_{\rho}^{2} \ll 1$. Comparing the terms linear in $t$, we find from (2.42) and (2.43) that $L_{9}^{r}=F_{\pi}^{2} / 2 M_{\rho}^{2} \simeq 7.3 \cdot 10^{-3}$, which is close to the phenomenological value of $L_{9}^{r}\left(M_{\rho}\right)$. The small discrepancy is due to the fact that VMD does not accurately give the vector radius, $\left\langle r^{2}\right\rangle_{\pi, \mathrm{VMD}}^{V}=6 / M_{\rho}^{2}=0.40$ $\mathrm{fm}^{2}$ while experimentally $<r^{2}>_{\pi}^{V}=0.439 \pm 0.008 \mathrm{fm}^{2}[31]$. 
This method has been generalized by Ecker et al. [32] and by Donoghue et al. [33]. They consider the lowest order effective theory of Goldstone bosons coupled to resonance fields $(\mathrm{R})$. These resonances are of vector $(\mathrm{V})$, axial-vector $(\mathrm{A})$, scalar $(\mathrm{S})$ and non-Goldstone pseudoscalar (P) type. For the latter category, only the $\eta^{\prime}$ is of practical importance (cf. eq(2.38)). The form of the pertinent couplings is dictated by chiral symmetry in terms of a few coupling constants which can be determined from data (from meson-meson and meson-photon decays). At low momenta, one integrates out the resonance fields. Since their couplings to the Goldstone bosons are of order $q^{2}$, resonance exchange produces terms of order $q^{4}$ and higher. Symbolically, this reads

$$
\int[d R] \exp \left(i \int d^{4} x \tilde{\mathcal{L}}_{\text {eff }}[U, R]\right)=\exp \left(i \int d^{4} x \mathcal{L}_{\text {eff }}[U]\right)
$$

So to leading order $\left(q^{4}\right)$, one only sees the momentum-independent part of the resonance propagators,

$$
\frac{1}{M_{R}^{2}-t}=\frac{1}{M_{R}^{2}}\left(1+\frac{t}{M_{R}^{2}-t}\right)
$$

and thus the $L_{i}^{r}\left(\mu \simeq M_{R}\right)$ can be expanded in terms of the resonance coupling constants and their masses. This leads to

$$
L_{i}^{r}(\mu)=\sum_{R=V, A, S, P} L_{i}^{\mathrm{Res}}+\hat{L}_{i}(\mu)
$$

with $\hat{L}_{i}(\mu)$ a remainder. For this scenario to make sense, one has to choose $\mu$ somewhere in the resonance region. A preferred choice is $\mu=M_{\rho}$ (as shown in Ref.[32], any value of $\mu$ between $500 \mathrm{MeV}$ and $1 \mathrm{GeV}$ does the job). As an example, let me show the result for the low energy constant $L_{3}^{r}\left(M_{\rho}\right)$

$$
\begin{aligned}
L_{3}^{r}=L_{3}^{V}+L_{3}^{S} & =-\frac{3}{4} \frac{G_{V}^{2}}{M_{V}^{2}}+\frac{1}{2} \frac{c_{d}^{2}}{M_{S}^{2}} \\
& =(-3.55+0.53) \cdot 10^{-3}
\end{aligned}
$$

As advocated, only the resonance masses $\left(M_{V}=770 \mathrm{MeV}, M_{S}=983 \mathrm{MeV}\right)$ and couplings appear $\left(\left|G_{V}\right|=53 \mathrm{MeV},\left|c_{d}\right|=32 \mathrm{MeV}\right)$. In table 1, we show the corresponding values for all low energy constants. It is apparent that the resonances almost completely saturate the $L_{i}^{r}$, with no need for additional contributions. This method of estimating $L_{i}^{r}$ is sometimes called QCD duality or the QCD version of VMD. In fact, it is rather natural that the higher lying hadronic states leave their imprints in the sector of the light pseudoscalars - as already stated, the typical resonance mass is the scale of new physics not described by the Godstone bosons.

Finally, I mention some other attempts to estimate these low energy constants. These are based on Nambu-Jona-Lasinio models [34], extended Nambu-Jona-Lasinio 
approaches [35], QCD coupled to constituent quarks [36] or solution to Coulomb gauge QCD in the ladder approximation [37]. All of this give results similar to the procedure described above. It is important to have such a tool since in many circumstances $\left(q^{6}\right.$ corrections, non-leptonic weak interactions) the number of coupling constants is too large to allow for a systematic phenomenological determination.

\begin{tabular}{|c|ccc|}
\hline$i$ & $L_{i}^{r}\left(M_{\eta}\right)$ & $L_{i}^{r}\left(M_{\rho}\right)$ & $L_{i}^{\mathrm{RES}}$ \\
\hline 1 & $0.9 \pm 0.3$ & $0.7 \pm 0.3$ & 0.6 \\
2 & $1.7 \pm 0.7$ & $1.3 \pm 0.7$ & 1.2 \\
$3^{*}$ & $-4.4 \pm 2.5$ & $-4.4 \pm 2.5$ & -3.0 \\
4 & $0.0 \pm 0.5$ & $-0.3 \pm 0.5$ & 0.0 \\
5 & $2.2 \pm 0.5$ & $1.4 \pm 0.5$ & 1.4 \\
6 & $0.0 \pm 0.3$ & $-0.2 \pm 0.3$ & 0.0 \\
$7^{*}$ & $-0.4 \pm 0.15$ & $-0.4 \pm 0.15$ & -0.3 \\
8 & $1.1 \pm 0.3$ & $0.9 \pm 0.3$ & 0.9 \\
9 & $7.4 \pm 0.2$ & $6.9 \pm 0.2$ & 6.9 \\
10 & $-5.7 \pm 0.3$ & $-5.2 \pm 0.3$ & -6.0 \\
\hline
\end{tabular}

Table 1: Low-energy constants for $S U(3)_{L} \times S U(3)_{R}$. The first two columns give the phenomenologically determined values at $\mu=M_{\eta}$ and $\mu=M_{\rho}$. The $L_{i}^{r}(i=1, \ldots, 8)$ are from ref.[14], the $L_{9,10}$ from ref.[43]. The ' '' denotes the constants which are not renormalized. The third column shows the estimate based on resonance exchange [32]. The constant $L_{10}$ has recently been reexamined by Donoghue and Holstein [261].

\subsection{Extensions}

Here I will briefly discuss some extensions of the chiral effective Lagrangian at next-to-leading order. I will be sketchy on these topics and the interested reader should consult the pertinent literature.

The first extension concerns the energy-momentum tensor in chiral theories. Let $\Theta_{\mu \nu}$ denote the energy-momentum tensor. Matrix elements of the type $<\pi \pi\left|\Theta_{\mu}^{\mu}\right| 0>$ appear in the decay of a light Higgs [40,49] (which is by now excluded experimentally) or in the $\pi \pi$ spectrum of the transitions $\psi^{\prime} \rightarrow \psi \pi \pi$ and $\Upsilon^{\prime} \rightarrow \Upsilon \pi \pi$ (when one makes use of the multipole expansion [38]). Donoghue and Leutwyler have enumerated the new terms and performed the pertinent renormalization procedure [39]. There are three new terms which couple the meson fields to the Ricci tensor and the curvature scalar plus 
three contact terms involving squares of the curvature. These have no meson matrix elements. The resulting energy-momentum tensor to order $q^{4}$ is given in Ref.[39],

$$
\Theta^{\mu \nu}=\Theta_{2}^{\mu \nu}+\Theta_{4, g}^{\mu \nu}+\Theta_{4, R}^{\mu \nu}+\mathcal{O}\left(q^{6}\right)
$$

where $\Theta_{4, g}^{\mu \nu}$ are the conventional terms arising from (2.29) but with Lorentz indices raised and lowered with $g_{\mu \nu}$ and $\Theta_{4, R}^{\mu \nu}$ is generated by the new couplings. The one particle matrix elements $\left\langle p^{\prime}\left|\Theta_{\mu \nu}\right| p\right\rangle$ (for $\pi, K, \eta$ ) are analysed. They contain two form factors, a scalar and a tensor since $\Theta_{\mu \nu}$ contains a spin-zero and a spin-two part. Comparison with a dispersive analysis of the scalar form factors [40] allows to pin down $L_{11}^{r}$ and $L_{13}^{r}, L_{12}^{r}$ is estimated via tensor $f_{2}$-exchange. The physical significance of these new couplings is the following. While $L_{12}^{r}$ determines the slope of the tensor form factor, the slope of the scalar form factor is given by $4 L_{11}^{r}+L_{12}^{r}$. The combination $L_{11}^{r}-L_{13}^{r}$ measures the flavour asymmetries generated by the quark masses. In this context, one can also make contact to the dilaton model of the conformal anomaly [41]. In that model, the breaking of the conformal symmetry is given in terms of an effective scalar field. The pertinent low-energy constants are saturated by this scalar and a comparison to the empirical values of $L_{11}^{r}$ and $L_{13}^{r}$ shows that the model can be considered semiquantitative at best. Translating the $L_{11}^{r}$ into the mass $M_{\sigma}$, one finds $560 \mathrm{MeV} \leq M_{\sigma} \leq$ $720 \mathrm{MeV}$, whereas the empirical value for $L_{13}^{r}$ leads to a larger scalar mass, $1000 \mathrm{MeV}$ $\leq M_{\sigma} \leq 1100 \mathrm{MeV}$.

The second extension I want to address concerns the axial $U(1)$ transformations. Even in the limit of vanishing quark masses, the $U(1)_{A}$ Noether current is not conserved

$$
i \partial^{\mu} J_{5 \mu}^{(0)}=\frac{3 \alpha_{s}}{8 \pi} G \tilde{G}+2 \sum_{q=u, d, s} m_{q} \bar{q} \gamma_{5} q
$$

Since $G \tilde{G}$ is a total divergence, one can define a gauge invariant current which generates $U(1)_{A}$ symmetry transformations in the chiral limit. A chiral rotation with its associated charge $\tilde{Q}_{5}$ shifts the $\theta$ vacuum of $\mathrm{QCD}, \exp \left\{i \alpha \tilde{Q}_{5}\right\}|0>=| \theta-6 \alpha>$ with $\alpha$ the angle of the chiral rotation. One therefore adds a $\theta$ source term to the QCD Lagrangian (cf. eq.(2.2)). In the basis where the quark mass matrix is diagonal QCD without sources is characterized by a vacuum angle $\theta(x)=\bar{\theta} . \theta$ can be added to the chiral Lagrangian in a straightforward manner [14,42]. The lowest order term is already given in eq.(2.9) provided one substitutes the external field $\chi$ by $\tilde{\chi}=\chi \exp (i \theta / 3)$. The $\theta$-dependence is then entirely contained in this new source (apart from a contact term). Clearly, $\tilde{\chi}$ is invariant under global $U(1)_{A}$ rotations. At next-to-leading order, one has the same terms as before (with $\chi \rightarrow \tilde{\chi}$ ) and five additional operators involving derivatives of $\theta$ plus higher order contact terms with no meson matrix elements. These terms are enumerated in ref.[42], for the later applications we only need one of them

$$
\mathcal{L}_{4}^{D \theta}=i L_{14} D_{\mu} \theta D^{\mu} \theta \operatorname{Tr}\left(\tilde{\chi}^{\dagger} U-U^{\dagger} \tilde{\chi}\right)+\ldots
$$


Finally one can also couple a pseudoscalar $S U(3)$-singlet field like e.g. the $\eta^{\prime}$. This is spelled out in detail in the original work of Gasser and Leutwyler [14] and will not be needed in what follows.

\section{LIGHT QUARK MASS RATIOS}

The current quark masses characterize the strength of the flavour symmetry breaking. They are small on typical hadronic scales as indicated by the fact that the $S U(3)$ predictions work fairly well. Expanding physical quantities in powers of the quark masses, the deviations from the symmetry limit are probing the quark masses. To lowest order, this is a clean method. The perturbation is of the form $\bar{q} \mathcal{M} q$ and forming ratios, one can eliminate the dependence on the unknown operator $\bar{q} q$. I will review how CHPT allows to fix the ratios of the light quark masses at leading and next-to-leading order. In the latter case, some model dependence enters as will be discussed later on. For a fairly comprehensive review on the history of the subject and the determinations of the absolute values of the quark masses, the reader is referred to Gasser and Leutwyler [3]. Also, as a cautionary remark, let me mention that the mass parameters appearing in the Lagrangian should not be confused with the so-called constituent masses. These represent the energy of quarks confined inside hadrons. They do not follow directly from QCD but rather from some effective model.

\subsection{Lowest order estimates}

The best place to investigate the light quark mass ratios is the spectrum of the pseudoscalar Goldstone bosons. As already discussed in section 2, their masses are directly proportional to the quark masses. Neglecting any electromagnetic and higher order corrections, the standard GMOR scenario [18] (see also ref.[44]) gives

$$
\begin{aligned}
M_{\pi^{+}}^{2} & =\left(m_{u}+m_{d}\right) B, \\
M_{K^{+}}^{2} & =\left(m_{u}+m_{s}\right) B, \\
M_{K^{0}}^{2} & =\left(m_{d}+m_{s}\right) B,
\end{aligned}
$$

which allows to extract

$$
\frac{m_{u}}{m_{d}}=0.66, \frac{m_{s}}{m_{d}}=20.1, \frac{\hat{m}}{m_{s}}=\frac{1}{24.2}
$$

with $\hat{m}=\left(m_{u}+m_{d}\right) / 2$ the average light quark mass. This estimate is, however, somewhat too naive. The charged pions and kaons are surrounded by a photon cloud which leads to a mass shift of order $e^{2}$ (isospin breaking). In the chiral limit, one can resort to Dashen's theorem [45] which states that the electromagnetic self-energy of a pseudoscalar meson $P$ is proportional to its charge $Q_{P}$,

$$
M_{P}^{2}=\left(M_{P}^{2}\right)_{\text {strong }}+e^{2} Q_{P} C+\mathcal{O}\left(e^{2} \mathcal{M}\right)
$$


As shown by Das et al. [46], the constant $C$ can be expressed as an integral over the vector and axial-vector spectral functions. The latter have been determined from the semi-leptonic $\tau \rightarrow \nu_{\tau}+n \pi$ decays [5]. Another way of fixing this constant is based on the empirical fact that for the pion the mass difference is almost entirely of electromagnetic origin, $\left(M_{\pi^{+}}-M_{\pi^{0}}\right)_{\text {strong }} \sim\left(m_{u}-m_{d}\right)^{2}$. This strong splitting is due to the $\pi^{0} \eta$ mixing which causes the neutral pion to become lighter than its charged partner. Numerically, this effect is tiny, $\sim 0.2 \mathrm{MeV}[3]$. Thus, the pion mass difference fixes the value of $C$, which for the kaons results in the following leading-order strong splitting:

$$
\left(M_{K^{0}}^{2}-M_{K^{+}}^{2}\right)_{\text {strong }}=M_{K^{0}}^{2}-M_{K^{+}}^{2}+M_{\pi^{+}}^{2}-M_{\pi^{0}}^{2}
$$

which amounts to $\left(M_{K^{0}}-M_{K^{+}}\right)_{\text {strong }}=(5.3-1.3) \mathrm{MeV}=4 \mathrm{MeV}[3]$ and modifies the quark mass ratios accordingly

$$
\frac{m_{u}}{m_{d}}=0.55, \frac{m_{s}}{m_{d}}=20.1, \frac{\hat{m}}{m_{s}}=\frac{1}{25.9}
$$

This is the celebrated result of Weinberg [47]. Before discussing the $\mathcal{O}\left(\mathcal{M}^{2}\right)$ corrections to these numbers, let us pause for a moment. The ratio $m_{u} / m_{d}$ is rather different from one, so why does one not see large isospin violations in physical processes? The answer lies in the fact that although $m_{d} \simeq 2 m_{u}$, both masses are so small compared to the typical hadronic scale that this effect is almost perfectly screened. One exception to be discussed later is the decay $\eta \rightarrow 3 \pi$, whose amplitude is proportional to $m_{d}-m_{u}$. Weinberg [47] has also proposed to look into isospin breaking effects in the pion-nucleon scattering lengths, but considering the present status of the empirical information, this does not seem to be a realistic proposal. However, before elaborating further on these topics, let us consider the corrections of order $\mathcal{M}^{2}$.

\subsection{Next-to-leading order estimates}

Before discussing various attempts to pin down the order $\mathcal{M}^{2}$ corrections to the quark mass ratios (3.5), we have to discuss one subtlety which arises at next-to-leading order. As noted by Kaplan and Manohar [48], the chiral symmetry does not differentiate between the conventional mass matrix $\mathcal{M}=\operatorname{diag}\left(m_{u}, m_{d}, m_{s}\right)$ (with $m_{u, d, s}$ real) or $\mathcal{M}^{\prime}$ defined by the eigenvalues

$$
\begin{aligned}
& m_{u}^{\prime}=\alpha_{1} m_{u}+\alpha_{2} m_{d} m_{s}, \\
& m_{d}^{\prime}=\alpha_{1} m_{d}+\alpha_{2} m_{u} m_{s}, \\
& m_{s}^{\prime}=\alpha_{1} m_{s}+\alpha_{2} m_{u} m_{d},
\end{aligned}
$$

with $\alpha_{1}, \alpha_{2}$ two arbitrary constants. As already noted, only the product $B \mathcal{M}$ (via the scalar field $s(x)$ ) enters the chiral Lagrangian and thus $\alpha_{1}$ merely renormalizes the value of $B$. The constant $\alpha_{2}$ enters at order $\mathcal{M}^{2}$ and contributes to $\mathcal{L}_{4}$. To be more specific, 
it contributes to the terms with two powers of $\chi$. One can therefore completely hide this symmetry by redefining

$$
B^{\prime}=B / \alpha_{1}, L_{6}^{\prime}=L_{6}-c, L_{7}^{\prime}=L_{7}-c, L_{8}^{\prime}=L_{8}+2 c .
$$

with $c=\left(\alpha_{2} / \alpha_{1}\right)\left(F^{2} / 32 B\right)$. This means that $\mathcal{L}_{2}+\mathcal{L}_{4}$ is invariant under the symmetry (3.7). Notice that the term proportional to $L_{5}^{r}$ is not affected since it only contains one power of the quark mass matrix. Furthermore, it should be stressed that this is not a symmtery of QCD but rather an artefact of the truncated chiral expansion (for a different view, see Ref.[42]). In any case, some theoretical arguments are necessary to overcome this ambiguity if one wants to extract the corrections of order $\mathcal{M}^{2}$ to the quark mass ratios.

Let us consider first the classical analysis of Gasser and Leutwyler [14]. The chiral expansion of the pseudoscalar masses at next-to-leading order reads

$$
\begin{aligned}
& M_{\pi}^{2}=\stackrel{\circ}{M}_{\pi}^{2}\left\{1+\mu_{\pi}-\frac{1}{3} \mu_{\eta}+2 \hat{m} K_{3}+K_{4}\right\}, \\
& M_{K}^{2}=\stackrel{\circ}{M}_{K}^{2}\left\{1+\frac{2}{3} \mu_{\eta}+\left(\hat{m}+m_{s}\right) K_{3}+K_{4}\right\}, \\
& M_{\eta}^{2}=\stackrel{\circ}{M}_{\eta}^{2}\left\{1+2 \mu_{K}-\frac{4}{3} \mu_{\eta}+\frac{2}{3}\left(\hat{m}+m_{s}\right) K_{3}+K_{4}\right\}+K_{5}+\stackrel{\circ}{M}_{\pi}^{2}\left\{-\mu_{\pi}+\frac{2}{3} \mu_{K}+\frac{1}{3} \mu_{\eta}\right\} .
\end{aligned}
$$

with

$$
\begin{aligned}
\mu_{P} & =\frac{M_{P}^{2}}{16 \pi^{2} F^{2}} \ln \left(M_{P} / \mu\right), \\
K_{3} & =2 \kappa\left(2 L_{8}^{r}-L_{5}^{r}\right), K_{4}=4\left(2 \hat{m}+m_{s}\right) \kappa\left(2 L_{6}^{r}-L_{4}^{r}\right), \\
K_{5} & =\frac{8}{9} \kappa^{2}\left(3 L_{7}+L_{8}^{r}\right), K_{6}=\kappa L_{5}^{r} / F, \\
K_{7} & =2 \kappa\left(2 \hat{m}+m_{s}\right) L_{4}^{r} / F, \kappa=4 B / F .
\end{aligned}
$$

neglecting terms of the order $m_{u}-m_{d}$. Inspired by the discussion of the lowest order mass ratios, one forms the dimensionless quantities

$$
\begin{aligned}
Q_{1} & =\frac{M_{K}^{2}}{M_{\pi}^{2}}=\frac{\hat{m}+m_{s}}{2 \hat{m}}(1+\Delta) \\
Q_{2} & =\frac{\left(M_{K^{0}}^{2}-M_{K^{+}}^{2}\right)_{\text {strong }}}{M_{K}^{2}-M_{\pi}^{2}}=\frac{m_{d}-m_{u}}{m_{s}-\hat{m}}(1+\Delta) .
\end{aligned}
$$

Remarkably, in both cases the correction $\Delta$ is the same ( for an explicit expression, see ref.[14]). Therefore, up to corrections of order $\mathcal{M}^{2}$ one can form a ratio independent of the low energy constants (which are hidden in the explicit value of $\Delta$ ):

$$
\left(\frac{m_{u}}{m_{d}}\right)^{2}+\frac{Q_{2}}{Q_{1}}\left(\frac{m_{s}}{m_{d}}\right)^{2}=1
$$


which defines an ellipsis with semi-axis 1 and $\sqrt{Q_{2} / Q_{1}}$, respectively. Making use of Dashen's theorem, this leads to $\sqrt{Q_{2} / Q_{1}}=23.6$. Therefore, eq.(3.11) constitutes a lowenergy theorem - any value of the mass ratios $m_{u} / m_{d}$ and $m_{s} / m_{d}$ must fulfill this elliptic constraint (modulo corrections to Dashen's theorem, see below). However, the actual values of these ratios depend on the value of the correction $\Delta$. This depends on the lowenergy constants $L_{5}^{r}$ and $L_{8}^{r}$. Redefinitions of $L_{5,8}^{r}$ allow one to move along the ellipse. In particular, one can choose $m_{u}=0$ which is favored in connection with the strong CPproblem [50] (see also the instanton gas calculation reported in ref.[51]). However, there are strong phenomenological constraints on the low-energy constants. First, the value of $L_{5}^{r}$ is rather narrowly pinned down by the coupling constant ratio $F_{K} / F_{\pi}=1.22 \pm 0.01$ [52] leading to the value given in table 1 (it is also consistent with scalar resonance exchange for $M_{S} \simeq 1 \mathrm{GeV}$ ). Concerning $L_{8}^{r}$, the situation is less favorable. Making use of the deviation from the Gell-Mann-Okubo relation (cf. eq.(3.8)), which involves the couplings $L_{5}^{r}, L_{7}$ and $L_{8}^{r}$ and is well known, Leutwyler [53] has proposed to estimate $L_{7}$ via $\eta^{\prime}$ exchange as given in eq(2.38). $L_{7}$ also enters the determination of the $\eta \eta^{\prime}$ mixing angle, which is empirically constrained to $\Theta_{\eta \eta^{\prime}}=(22 \pm 4)^{\circ}$ [54]. This allows to fix $L_{8}^{r}$. The elliptic constraint together with the one from the $\eta \eta^{\prime}$ mixing leads to

$$
\frac{m_{u}}{m_{d}}=0.55 \pm 0.12, \frac{m_{s}}{m_{d}}=20 \pm 2.2,
$$

rather consistent with Weinberg's estimate (3.5). A further check comes from the baryon mass spectrum. Gasser [55] and Gasser and Leutwyler [3] have analyzed the nextto-leadig order quark mass corrections which scale as $\mathcal{M}^{3 / 2}$. Combining the isospin breaking mass splittings $m_{p}-m_{n}, m_{\Sigma^{+}}-m_{\Sigma^{-}}$and $m_{\Xi^{0}}-m_{\Xi^{-}}$one arrives at

$$
R=\frac{m_{s}-\hat{m}}{m_{d}-m_{u}}=43.5 \pm 3.2
$$

This result for $R$ is also supported by examining the $\rho \omega$ splitting at next-to-leading order [3]. Imposing this constraint on the results (3.12), one ends up with

$$
\frac{m_{u}}{m_{d}}=0.56 \pm 0.06, \frac{m_{s}}{m_{d}}=20 \pm 2, \frac{\hat{m}}{m_{s}}=\frac{1}{25.6 \pm 2.0}
$$

which shows that the meson spectrum (consistently with the baryon mass splittings) determines the quark mass ratios rather accurately. This is a consistent picture. However, what is definitively missing is a better treatment of the corrections to Dashen's theorem.

Donoghue and Wyler [42] have taken a somewhat different path to get a handle on the second order corrections to the quark mass ratios. They were inspired by the instanton gas calculation of Choi et al. [51]. In that calculation, in which an originally massless up quark travels through the instanton gas, it acquires an effective mass,

$$
m_{u}^{\mathrm{eff}} \sim m_{d} m_{s} \mathrm{e}^{i \theta}
$$


due to quantum effects ('t Hooft's six-fermion term which has been shown to be of utmost importance in understanding the problem of flavor mixing in theories with quark degrees of freedom [56]). Notice that the vacuum angle $\theta$ enters the game. The $\theta$ dependence is the main ingredient in the calculation of ref.[42] to overcome the KaplanManohar ambiguity. The basic observation is that the operator $G \tilde{G}$, which follows from $\mathcal{L}_{\mathrm{QCD}}$ after variation by $\theta$, is immune to the reparametrization invariance of the quark masses. However, since one can not directly measure the $\theta$-dependence from strong CPviolating effects, one has to resort to the multipole expansion for heavy quark systems [38]. The decays $V \rightarrow V+M$ with $V=\Psi$ or $\Upsilon$ and $M=\pi^{0}, \eta, 3 \pi$ are determined by the operator $G \tilde{G}$ to leading order in the heavy quark expansion. Taking ratios, one can eliminate almost all model dependence. The strength of $G \tilde{G}$ can be parametrized in terms of the number $r_{G \tilde{G}}$,

$$
\begin{aligned}
& r_{G \tilde{G}}=\frac{<0|G \tilde{G}| \pi^{0}>}{<0|G \tilde{G}| \eta>}=\frac{3 \sqrt{3}}{4}\left(\frac{m_{d}-m_{u}}{m_{s}-\hat{m}}\right) \frac{F_{\eta}}{F_{\pi}} \\
& \times\left\{1-\frac{32 B}{F_{\pi}^{2}}\left(m_{s}-\hat{m}\right)\left(L_{7}+L_{8}^{r}\right)+\frac{4 L_{14}^{r}}{F_{\pi}^{2}}\left(M_{\pi}^{2}-M_{\eta}^{2}\right)-\left(3 \mu_{\pi}-2 \mu_{K}-\mu_{\eta}\right)\right\}
\end{aligned}
$$

To arrive at this result, one uses $\partial \mathcal{L}_{\text {eff }} / \partial \theta \sim G \tilde{G}$ and sandwiches the operator between the pertinent states. Clearly, all the terms in $\mathcal{L}_{\text {eff }}$ which contain $\tilde{\chi}=\chi \exp (i \theta / 3)$ can contribute and there is also the term proportional to $L_{14}^{r}$ (cf. eq.(2.50)) which is linear in $\theta$. The first term on the right hand side of (3.16) agrees with the result of Novikov et al. [57]. One can furthermore form one particular combination of the mass ratios which is free of chiral logarithms (contained in the $\mu_{P}$ ) and only depends on one low-energy constant $[42]$

$\frac{m_{d}-m_{u}}{m_{d}+m_{u}} \frac{m_{s}+\hat{m}}{m_{s}-\hat{m}}=\frac{4\left(F_{K}^{2} M_{K}^{2}-F_{\pi}^{2} M_{\pi}^{2}\right)}{3 \sqrt{3} F_{\pi}^{2} M_{\pi}^{2}} \frac{F_{\pi}}{F_{\eta}} r_{G \tilde{G}}\left[1-\Delta_{G M O}\right]\left[1+\frac{4 L_{14}^{r}}{F \pi^{2}}\left(M_{\eta}^{2}-M_{\pi}^{2}\right)\right]$

so that one has to determine $r_{G \tilde{G}}$ and $L_{14}^{r}$ to get this mass ratio. The first quantity can be determined from

$$
\frac{\Gamma\left(V^{\prime} \rightarrow V \pi^{0}\right)}{\Gamma\left(V^{\prime} \rightarrow V \eta\right)}=r_{G \tilde{G}}^{2}\left(\frac{p_{\pi}}{p_{\eta}}\right)^{3}
$$

with $p_{\pi}\left(p_{\eta}\right)$ the momentum of the $\pi(\eta)$. The data on the decays $\Psi^{\prime} \rightarrow J / \Psi+\pi^{0}$ and $\Psi^{\prime} \rightarrow J / \Psi+\eta$ lead to $r_{G \tilde{G}}=0.043 \pm 0.0055$. Furthermore, one can use the principle of resonance saturation to estimate $L_{14}^{r}$. The authors of ref.[42] give the following limits: $0 \leq 4 L_{14}^{r} / F_{\pi}^{2} \leq M_{\eta^{\prime}}^{2}$. Combining pieces and adding the errors (theoretical and empirical) in quadrature, this leads to

$$
\frac{m_{d}-m_{u}}{m_{d}+m_{u}} \frac{m_{s}+\hat{m}}{m_{s}-\hat{m}}=0.59 \pm 0.11
$$


or equivalently (since $\left.m_{s} \gg \hat{m}\right)$ :

$$
\frac{m_{u}}{m_{d}}=0.30 \pm 0.07
$$

This result gives further credit to the notion that $m_{u}$ is indeed unequal zero, but its rather large deviation to the value given in (3.14) should be noted. Such an effect can only occur if there are substantial corrections to Dashen's theorem, much bigger than assumed in the calculation of Leutwyler [53]. This will be discussed below. The estimate of Donoghue and Wyler can be criticied on two grounds. First, it corresponds to a value of $L_{7}$ which is not in particular good agreement with phenomenology. Second, it is not clear that the multipole expansion is justified in the $\Psi^{\prime}(\Psi)$ system. A measurement for the upsilon system would be very much needed to calrify the uncertainties inflicted by the heavy quark expansion on the result (3.20).

Donoghue, Holstein and Wyler [58] have extended this latter analysis by considering also the $\eta \rightarrow 3 \pi$ decay and a model to estimate the corrections to Dashen's theorem (see also ref.[59] for an earlier attempt). They consider the particular mass ratio

$$
R_{1}=\frac{m_{d}-m_{u}}{m_{s}-\hat{m}} \frac{2 \hat{m}}{m_{s}+\hat{m}}=\left(\frac{M_{\pi}}{M_{K}}\right)^{2} \frac{\left(M_{K^{0}}^{2}-M_{K^{+}}^{2}\right)_{\text {strong }}}{M_{K}^{2}-M_{\pi}^{2}}
$$

which is related to the strong kaon mass difference and also appears in the chiral expansion of the decay amplitude $\eta \rightarrow \pi^{+} \pi^{0} \pi^{-}$. The latter has been worked out to one loop order by Gasser and Leutwyler [60]. The matrix element for $\eta \rightarrow 3 \pi$ can be parametrized by one single function of the pertinent Mandelstam variables, denominated $A(s, t, u)$. To leading order, the chiral expansion of $A(s, t, u)$ reads

$$
A(s, t, u)=-\frac{\left(m_{d}-m_{u}\right) B}{3 \sqrt{3} F_{\pi}^{2}}\left\{1+\frac{3\left(s-s_{0}\right)}{M_{\eta}^{2}-M_{\pi}^{2}}+\mathcal{O}\left(q^{2}\right)+\mathcal{O}\left(e^{2} \frac{m_{s}-\hat{m}}{m_{u}-m_{d}}\right)\right\}
$$

with $s_{0}=M_{\pi}^{2}+M_{\eta}^{2} / 3$ the center of the Dalitz plot. While current algebra (tree level) predicts $\Gamma\left(\eta \rightarrow \pi^{+} \pi^{0} \pi^{-}\right)=66 \mathrm{eV}$, the one-loop calculation leads to $(160 \pm 50) \mathrm{eV}$ (the uncertainty is an estimate of the higher order terms in the chiral expansion). Notice that these values are in disagreement with the empirical result of $(281 \pm 29) \mathrm{eV}$. The large enhancement of the one-loop result over the tree level prediction is essentially an unitarity effect, i.e. an effect of the strong pionic final state interactions in the $S$-wave. This was originally predicted by Roiesnel and Truong [15] who found an even larger discrepancy (see the discussion in ref.[60]). Notice also that these uncertainties essentially drop out in the ratio $\Gamma\left(\eta \rightarrow 3 \pi^{0}\right) / \Gamma\left(\eta \rightarrow \pi^{+} \pi^{0} \pi^{-}\right)=1.51,1.43$ for current algebra and one loop CHPT [60], respectively. The emiprical value is $1.35 \pm 0.04$, comparable to the one loop prediction. The authors of ref.[58], however, turn the argument around. They use the empirical rate for $\eta \rightarrow \pi^{+} \pi^{0} \pi^{-}$to fix the strong kaon mass difference since $\Gamma\left(\eta \rightarrow \pi^{+} \pi^{0} \pi^{-}\right)$fixes $R_{1}(3.21)$. This gives

$$
\left(\Delta M_{K}^{2}\right)_{\text {strong }}=\left(M_{K^{0}}^{2}-M_{K^{+}}^{2}\right)_{\text {strong }}=2 M_{K} \cdot(7.0 \mathrm{MeV}) .
$$


This is considerably different from the result based on Dashen's theorem, $\left(\Delta M_{K}^{2}\right)_{\text {strong }}=$ $2 M_{K} \cdot(5.3 \mathrm{MeV})$ (a 30 per cent deviation). To further support this argument, they also consider a model to independently estimate the corrections to Dashen's theorem. It is based on the empirical observation that vector meson dominated form factors in the Born diagrams give a rather accurate description of the electromagnetic pion mass difference [16,32,33] (see fig.3). Beyond leading order and making use of the Weinberg sum rules (which is necessary to cancel the divergent pieces) this leads to

Fig. 3: Electromagnetic self-energy of pseudoscalars to order $e^{2}$ in the resonance exchange picture. The wiggly lines denote photons, the double solid lines vector or axial mesons. Tadpole diagrams are not shown.

$$
\Delta M_{\pi}^{2}=2 M_{\pi} \cdot(6.3 \mathrm{MeV})
$$

using the physical mass of the $A_{1}$ meson. The result (3.24) is considerably larger than the empirical value $\Delta M_{\pi}^{2}=2 M_{\pi} \cdot(4.6 \mathrm{MeV})$. The lowest order result, however, is within a few per cent of the empirical value, $\Delta M_{\pi}^{2}=2 M_{\pi} \cdot(4.7 \mathrm{MeV})[32]$. This sheds some doubt on the accuracy of this approach. To leading order, the formalism used to arrive at (3.24) respects Dashen's theorem but predicts a strong violation of it at next-toleading order, $\left(\Delta M_{K}^{2}\right)_{\mathrm{em}}=1.78\left(\Delta M_{\pi}^{2}\right)_{\mathrm{em}}$. This large deviation from one is essentially due to the kaon propagator in the Born terms (cf. fig.3) and thus the strong kaon mass difference is affected,

$$
\left(\Delta M_{K}^{2}\right)_{\text {strong }}=2 M_{K} \cdot(6.3 \pm 0.1 \mathrm{MeV})
$$

which agrees within 10 per cent with the number (3.23) extracted from the $\eta \rightarrow 3 \pi$ decay. Assuming furthermore resonance saturation for the low-energy constants $L_{7}$ and $L_{14}^{r}$ ( $\eta^{\prime}$ exchange), the authors of ref.[58] can also determine the mass ratios $2 \hat{m} /\left(\hat{m}+m_{s}\right)$ 
and $\left(m_{d}-m_{u}\right)\left(m_{s}+\hat{m}\right) /\left(m_{d}+m_{u}\right)\left(m_{s}+\hat{m}\right)$ (from the meson masses and the decays $\left.\Psi^{\prime} \rightarrow J / \Psi+\pi, \eta\right)$ and arrive at

$$
\frac{\hat{m}}{m_{s}}=\frac{1}{31}, \frac{m_{d}-m_{u}}{m_{s}}=\frac{1}{29}, \frac{m_{d}-m_{u}}{m_{d}+m_{u}}=0.59
$$

or

$$
\frac{m_{u}}{m_{d}}=0.26
$$

This value for the ratio of the up and down quark masses is consistent with the one given in (3.20). While this set of mass ratios has the virtue of giving a correct $\eta \rightarrow 3 \pi$ amplitude, which is a notorious problem in CHPT, there are various aspects which have to be looked at in more detail. First, from (3.26) one deduces $R \simeq 28$ which is four standard deviations off the value extracted from the baryon spectrum (see also the discussion at the end of this section). Furthermore, it is not obvious that the one-loop result for the amplitude $A(\eta \rightarrow 3 \pi)$ should be used for fixing the mass ratio $R_{1}$. As we will see from the discussion of the scalar form factor in section 4 , strong pionic final state interactions cause the failure of the one loop approximation close to two-pion threshold. For $\eta \rightarrow 3 \pi$, one deals with $\sqrt{s} \approx 550 \mathrm{MeV}$. Finally, the large next-to-leading order corrections to the pion mass difference put a question mark on the convergence of this approach.

From the discussion so far it should have become clear that the calculations of the quark mass ratios at next-to-leading order involve some model-dependence. It is therefore necessary to investigate as many constraints as possible (meson masses, baryon masses, $\eta \rightarrow 3 \pi$, corrections to Dashen's theorem and alike) to arrive at a consistent picture of the quark mass ratios at order $\mathcal{M}^{2}$. If the modifications to $m_{u} / m_{d}$ and $\hat{m} / m_{s}$ are indeed as large as indicated by (3.20) or (3.26), it is not obvious why one should stop at next-to-leading order. The set of quark mass ratios (3.14) has the virtue of showing stability against inclusion of the quark masses as perturbations. At present, I consider it as the most consistent estimation of the light quark mass ratios.

There is some additional evidence which gives further credit to the constraint from the baryon sector, i.e. the value of $R$ (3.13). This work is based on so-called second order "flavor" perturbation theory [61]. It extends the work of Gell-Mann, Okubo and others [17]. It differs from CHPT in that the flavor symmetric part of the quark mass term $\left(m_{u}+m_{d}+m_{s}\right)(\bar{u} u+\bar{d} d+\bar{s} s) / 3$ is already contained in the unperturbed Hamiltonian. The basic idea is that in this way one includes already most of the (large) $\mathcal{M}^{3 / 2}$ corrections of CHPT and thus deals with smaller perturbations. If one works out the flavor perturbations to second order, one finds [61]:

$$
R=\frac{3 m_{\Lambda}+m_{\Sigma}-2 m_{N}-2 m_{\Xi}}{2 \sqrt{3} m_{T}+\left(m_{n}-m_{p}\right)+\left(m_{\Xi^{0}}-m_{\Xi^{-}}\right)} .
$$

Here, $m_{T}$ is the $\Lambda-\Sigma^{0}$ transition mass. Its physical effect is that it produces a small mixing $\epsilon$ of the mass-diagonal fields, $\epsilon=m_{T} /\left(m_{\Sigma}-m_{\Lambda}\right)$. Empirically, it is not well 
determined. It could be pinned down from a precision measurement of the difference in the $p K^{-} \rightarrow \Lambda \eta$ and $n \bar{K}^{0} \rightarrow \Lambda \eta$ cross sections. At present, one can only deduce a (conservative) lower limit of $R \geq 38 \pm 11$. A similar result can also be obtained from the splittings in the decuplet. Finally, if one assumes that the first order result lies as close as possible to the family of second order solutions, one finds $R=48 \pm 5$, quite consistent with the value given in eq.(3.13). It is certainly desirable to have such extra information to strengthen our understanding of the ratios of the light quark masses. Some arguments which seem to support the notion of a much smaller ratio $m_{s} / \hat{m}$ are discussed in section 4.2 .

\section{THE MESON SECTOR - SELECTED TOPICS}

In this section, I discuss a few selected topics of applying CHPT in the meson sector. Clearly, space forbids to account for less than a small fraction of the many existing predictions and their comparison to the data. In section 8, additional references concerning some of the neglected topics are given.

Here, I will first discuss the classical topic of pion-pion scattering within the one loop approximation. This is an important topic since, as will be discussed, it allows to directly test our understanding of the mechanism of quark condensation in the vacuum. I also discuss briefly the related subject of $\pi K$ scattering. Then, the question of the range of applicability of the chiral expansion is discussed. Various schemes to extend the energy range are critically reviewed. I also sketch the extenstion of the chiral Lagrangian at next-to-leading order to include the non-leptonic weak interactions with particular emphasis on the decay modes $K \rightarrow 2 \pi$ and $K \rightarrow 3 \pi$.

\section{1. $\pi \pi$ scattering}

The scattering of pions is in a sense the purest reaction to test our understanding of the low energy sector of QCD. It involves only the pseudoscalar Goldstone bosons and their dynamics. Also, for the reaction $\pi \pi \rightarrow \pi \pi$ one can restrict oneself to the sector of $S U(2) \times S U(2)$, where the quark mass corrections are expected to be very small. To be more specific, consider the process $\pi^{a}\left(p_{a}\right)+\pi^{b}\left(p_{b}\right) \rightarrow \pi^{c}\left(p_{c}\right)+\pi^{d}\left(p_{d}\right)$, for pions of isospin ' $a, b, c, d$ ' and momenta $p_{a, b, c, d}$. The conventional Mandelstam variables are defined via $s=\left(p_{a}+p_{b}\right)^{2}, t=\left(p_{a}-p_{c}\right)^{2}$ and $u=\left(p_{a}-p_{d}\right)^{2}$ subject to the constraint $s+t+u=4 M_{\pi}^{2}$. The scattering amplitudes can be expressed in terms of a single function, denoted $A(s, t, u)$ :

$$
T^{c d ; a b}(s, t, u)=A(s, t, u) \delta^{a b} \delta^{c d}+A(t, s, u) \delta^{a c} \delta^{b d}+A(u, t, s) \delta^{a d} \delta^{b c}
$$

for the two flavor case. The chiral expansion of $A(s, t, u)$ takes the form

$$
A(s, t, u)=A^{(2)}(s, t, u)+A^{(4)}(s, t, u)+\mathcal{O}\left(q^{6}\right)
$$


where $A^{(m)}$ is of order $q^{m}$ and the symbol $\mathcal{O}\left(q^{6}\right)$ denotes terms like $s^{3}, s^{2} t, s t^{2}, \ldots$ The explicit form of the tree level amplitude was first given by Weinberg [62]. It can be easily read off from eq.(2.9) by expanding in powers of the pion field and collecting the terms proportional to $\pi^{4}$,

$$
A^{(2)}(s, t, u)=\frac{s-M_{\pi}^{2}}{F_{\pi}^{2}}+\mathcal{O}\left(q^{4}\right),
$$

where it is legitimate to use the physical values of the pion mass and decay constant since the differences to their lowest order values is of order $q^{4}$. The next-to-leading order term has been worked out by Gasser and Leutwyler [13,63] (cf. fig.2). One splits it into unitarity corrections (denoted $B(s, t, u)$ ) and tree and tadpole contributions (denoted $C(s, t, u)$ ). The explicit form reads (I use the one given in ref.[64]):

$$
\begin{aligned}
A^{(4)}(s, t, u)= & B(s, t, u)+C(s, t, u) \\
B(s, t, u)= & \frac{1}{6 F_{\pi}^{4}}\left[3\left(s-M_{\pi}^{4}\right) \bar{J}(s)+\left\{\left[t(t-u)-2 M_{\pi}^{2} t+4 M_{\pi}^{2} u-2 M_{\pi}^{4}\right] \bar{J}(t)+(t \leftrightarrow u)\right\}\right] \\
C(s, t, u)= & \frac{1}{96 \pi^{2} F_{\pi}^{4}}\left\{2\left(\bar{\ell}_{1}-4 / 3\right)\left(s-2 M_{\pi}^{2}\right)^{2}+\left(\bar{\ell}_{2}-5 / 6\right)\left[s^{2}+(t-u)^{2}\right]\right. \\
& \left.+12 M_{\pi}^{2} s\left(\bar{\ell}_{4}-1\right)-3 M_{\pi}^{4}\left(\bar{\ell}_{3}+4 \bar{\ell}_{4}-5\right)\right\} \\
\bar{J}(z)= & -\frac{1}{16 \pi^{2}} \int_{0}^{1} d x \ln \left[1-z x(x-1) / M_{\pi}^{2}\right] .
\end{aligned}
$$

In the chiral limit, one recovers the form of $A(s, t, u)$ first given by Lehmann [65]. Unitarity and analyticity force the appearance of the loop terms which contain two unknown scales. These can be expressed in terms of the low energy constants $\bar{\ell}_{1,2}$ [13]. The polynomial term $C(s, t, u)$ contains two further constants related to the shift of $F_{\pi}$ and $M_{\pi}$ away from their lowest order values. An explicit expression of $A^{(4)}(s, t, u)$ in terms of the $S U(3)$ representation can be found in ref.[66].

For comparison with the data, one decomposes $T^{c d ; a b}$ into amplitudes of definite total isospin $(I=0,1,2)$ and projects out partial-wave amplitudes $T_{l}^{I}(s)$,

$$
T_{l}^{I}(s)=\frac{\sqrt{1-4 M_{\pi}^{2} / s}}{2 i}\left[\exp \left\{2 i\left[\delta_{l}^{I}(s)+i \eta_{l}^{I}(s)\right]\right\}-1\right]
$$

with $s=4\left(M_{\pi}^{2}+q^{2}\right)$ in the c.m system, $l$ denotes the total angular momentum of the two-pion system. The phase shifts $\delta_{l}^{I}(s)$ are real and the inelasticities $\eta_{l}^{I}(s)$ set in at four-pion threshold. They are, however, negligible below $\bar{K} K$ threshold (below which they are a three-loop effect of order $\left.q^{8}\right)$. Near threshold $\left(s=4 M_{\pi}^{2}\right)$ the partial-wave amplitudes take the form

$$
\operatorname{Re} T_{l}^{I}=q^{2 l}\left\{a_{l}^{I}+q^{2} b_{l}^{I}+\mathcal{O}\left(q^{4}\right)\right\}
$$


The coefficients $a_{l}^{I}$ are called scattering lengths, the $b_{l}^{I}$ are the range parameters. It was already observed by Weinberg [62] that the scattering lengths resulting from the tree level were much smaller than naively expected. This is also borne out in the one loop calculation [13]. Let me now concentrate on the isospin-zero $S$-wave scattering length $a_{0}^{0}$. The tree level prediction is $a_{0}^{0}=7 M_{\pi}^{2} / 32 \pi F_{\pi}^{2}=0.16$ [62] and to one loop order, one can derive the following low energy theorem [13],

$a_{0}^{0}=\frac{7 M_{\pi}^{2}}{32 \pi F_{\pi}^{2}}\left\{1+\frac{1}{3} M_{\pi}^{2}<r^{2}>_{S}^{\pi}-\frac{M_{\pi}^{2}}{672 \pi^{2} F_{\pi}^{2}}\left(15 \bar{\ell}_{3}-353\right)+\frac{25}{4} M_{\pi}^{4}\left(a_{2}^{0}+2 a_{2}^{2}\right)+\mathcal{O}\left(q^{6}\right)\right\}$

which involves the scalar radius of the pion, $\left\langle r^{2}>_{S}^{\pi} \simeq 0.7 \mathrm{fm}^{2}\right.$ (see also section 4.4), the low energy constant $\bar{\ell}_{3}$ and the $D$-wave scattering lenghts $a_{2}^{0}$ and $a_{2}^{2}$. Taking the latter from Petersen [67] and allowing for conservative errors on the scalar radius and $\bar{\ell}_{3}$, one arrives at $[13,63]$

$$
a_{0}^{0}=0.20 \pm 0.01 \text {. }
$$

One makes two important observations. First, there is a 25 per cent correction to the tree result (which is due to the fact that the non-analytic term of the type $M_{\pi}^{2} \ln M_{\pi}^{2}$ has a large coefficient). Second, the theoretical uncertainty is rather small. This is mostly due to the fact that the poorly known $\bar{\ell}_{3}$ is multiplied by a tiny factor. We will come back to this important result in section 4.2. For a more detailed account of the threshold behaviour of the other partial waves, see refs.[13,63].

If one now increases the energy, there are two main issues to address. First, how does the truncated chiral series (4.2) compare with the data and, second, at what energy do the one-loop corrections become so large that one loop approximation can not be trusted any more? Before addressing these issues, I have to discuss an ambiguity which arises in the extraction of the phase shifts from eq.(3.6). One can either use [64]

$$
\delta_{l}^{I}=\operatorname{Re} T_{l}^{I(2)}+\operatorname{Re} T_{l}^{I(4)}+\mathcal{O}\left(q^{6}\right)
$$

or

$$
\delta_{l}^{I}=\arctan \left(\operatorname{Re} T_{l}^{I}\right)+\mathcal{O}\left(q^{6}\right) .
$$

The difference between these two forms only shows up at order $q^{6}$ and therefore gives an estimate about the relative importance of these higher order terms (in what follows, I will mostly use the definition (4.9)). In fig.4a,b,c the phase shifts $\delta_{0}^{0}(s), \delta_{1}^{1}(s)$ and $\delta_{0}^{2}(s)$ are shown, respectively [64]. For comparison, the tree result and the existing data are shown together with a band of Roy equation fits $[68,69]$ (the latter impose unitarity and analyticity and, obviously, some of the data are in conflict with these fundamental principles). At the time the Roy equation program was carried out [67,69], the $S$-wave scattering length, which is a fundamental input, was not known. The band indicated in the figures corresponds to $0.17 \leq a_{0}^{0} \leq 0.30$. Before discussing the results, let me point out that for $\delta_{0}^{0}$ one has additional information in the threshold region from $K_{\ell 4}$ 
Fig. 4: $\pi \pi$ scattering phase shifts $\delta_{0}^{0}(a), \delta_{1}^{1}(b)$ and $\delta_{0}^{2}(c)$, in order. The dashed line gives the tree result and the dashed-dotted the one-loop prediction. Also shown is the Roy equation band as discussed in the text. The data can be traced back from ref.[64]. In (a), the double-dashed line corresponds to the one-loop result based on eq.(4.10). 
decays. The energy where the one-loop contribution $\delta_{l}^{I(4)}$ is half as big as the tree level prediction (in magnitude) is indicated by the shaded vertical line. Notice that this critical energy is different for the various channels, $\sqrt{s}=450 \mathrm{MeV}, 480 \mathrm{MeV}$ and $470 \mathrm{MeV}$, in order. Beyond these energies, the truncated chiral expansion becomes unreliable. In the isospin-zero $S$-wave (fig.4a), the data below $\sqrt{s}=600 \mathrm{MeV}$ are poor (with the exception of the ones from $K_{\ell 4}$ decays). Notice that both representations (4.9) and (4.10) stay within the Roy equation band up to the critical energy. Below 400 $\mathrm{MeV}$, they are virtually identical. In the $P$-wave, the well-known $\rho$ resonance shrinks the Roy equation band to a narrow line. The chiral prediction follows it up to $500 \mathrm{MeV}$. Clearly, it is not possible to describe the resonance behaviour by this method. This is the natural barrier to the theory of pseudoscalars only already mentioned before. In the exotic $S$-wave $(I=2)$, the tree and one-loop contributions are of opposite sign. This leads to a stronger sensitivity of the chiral predictions to the actual values of the low-energy constants (see the shaded area in fig.4c, which corresponds to a change of $\ell_{4}$ by one unit. The central value of $\ell_{4}$ gives the lower rim of this area.)

It was noted in ref.[64] that the phase of the parameter $\epsilon^{\prime}$, which measures direct $\mathrm{CP}$-violation in the kaon system, can nevertheless be extracted with rather good accuracy (see also refs.[70,71]). It is defined via [72]

$$
\Phi\left(\epsilon^{\prime}\right)=\left.\left(\delta_{0}^{2}-\delta_{0}^{0}\right)\right|_{s=M_{K^{0}}^{2}}+\frac{\pi}{2}
$$

(see also section 4.5). One therefore needs the $S$-wave phase shifts at $\sqrt{s}=M_{K^{0}}=494$ $\mathrm{MeV}$, i.e. at an energy where the one-loop coorections are already large. However, in the difference these corrections largely cancel and one finds

$$
\Phi\left(\epsilon^{\prime}\right)=(45 \pm 6)^{\circ}
$$

to be compared with the tree result of $53^{\circ}$. The uncertainty of $6^{\circ}$ is a combination of the uncertainties in the low energy constants and the higher order contributions. Ochs [73] has recently reanalyzed results of phase shifts from $\pi^{-} p \rightarrow n \pi^{-} \pi^{+}$for energies above $600 \mathrm{MeV}$. Using Roy equation methods, he finds $\Phi\left(\epsilon^{\prime}\right)=(43 \pm 6)^{\circ}$, in good agreemnet with the chiral prediction. This is an important result since it essentially pins down this parameter. Attempts to extract $\Phi\left(\epsilon^{\prime}\right)$ from the various $K \rightarrow 2 \pi$ modes are hampered by the fact that there are large corrections from isospin breaking which have not yet been investigated in full detail (some references can be traced back from ref.[64]).

Donoghue et al. [74] have taken a different point of view on the one-loop results. They used the available data on the $S, P, D$ partial-wave amplitudes in the range of energies from threshold to $1 \mathrm{GeV}$ to determine the low energy constants $\bar{\ell}_{1,2}$ by a best global fit to these data. This leads to values which are somewhat different form the standard ones $[13,14]$. However, from the discussion before it should be clear that such a fit is not quite legitimate since the one-loop corrections are too large beyond 500 
$\mathrm{MeV}$. While the authors of ref.[74] find a generally satisfactory description of the data up $700 \ldots 800 \mathrm{MeV}$, it is not clear what one learns from such a comparison. Notice also that the partial-wave amplitudes are bounded in magnitude by unitarity and therefore a much less sensitive probe of deviations than the phase shifts.

There is furthermore a whole set of articles in which the one-loop results are extended to higher energies based on unitarization schemes or explicit resonance exchanges. These proposals will be discussed in section 4.4. Let me now return to the importance of the $S$-wave scattering lengths.

\subsection{Testing the mode of quark condensation}

So far, we have assumed the standard scenario in which $<0|\bar{u} u| 0>$ is the order parameter of the spontaneous chiral symmetry breaking and the constant $B=-<$ $0|\bar{u} u| 0>/ F^{2}$ is so large $(\sim 1 \mathrm{GeV})$ that the first term in the quark mass expansion of the pseudoscalar masses $(B \mathcal{M})$ dominates (this is also the point of view I subscribe, it is backed by lots of phenomenological and some theoretical arguments, see below). However, this notion has been challenged already in the seventies [75,76]. It was claimed that the possibility $M_{P}^{2} \sim \mathcal{M}^{2}$ fits the data as well (in that case, $B=0$ in the chiral limit). Some recent work along these lines has been done by Fuchs et al. [77], who argued that the Goldberger-Treiman discrepancies in the baryon octet (the deviations from the GT relations, which are exact in the chiral limit) lead to an upper bound for the quark mass ratio $m_{s} / \hat{m} \leq 10.6 \pm 4.2$. This is more than 3 standard deviations off the standard value (3.14) (for earlier work on this problem, see the review by Dominguez [78]). The same authors have recently proposed a modified CHPT [79] to account for this. Their starting point is the claim that a scenario in which $B$ is small, $B \sim \Lambda_{\mathrm{QCD}} \sim$ $F_{\pi} \sim 100 \ldots 200 \mathrm{MeV}$ is as natural as the large $B$ case. Then, the ratio $m_{s} / B$ is of order one and one has to modify the chiral power counting. Apart from the small external momenta, one has to consider the quark mass matrix $\mathcal{M}$ and the parameter $B$ simultaneously as small quantities, with the ratio $m_{s} / B$ kept fixed. It is then natural to assign the external field $\chi$ the dimension one. The effective Lagrangian takes the form:

$$
\mathcal{L}_{\mathrm{eff}}=\sum_{l, m, n} B^{l} \mathcal{L}_{m, n}^{l}=\sum_{N} \mathcal{L}^{(N)}
$$

with $m$ covariant derivatives, $n$ quark mass insertions and $l$ factors of $B$ so that $l+m+$ $n=N$ (with $l, m, n$ integers). In the standard case, $m+2 n=N$. This modifies the leading term of order $q^{2}$ and new terms at order $q^{3}$ appear (for explicit expressions, see ref.[79]). The leading non-analytic contributions are still of order $q^{4}$, i.e. at next-tonext-to leading order in the modified chiral expansion.

The best place to test this scenario is indeed $\pi \pi$ scattering in the threshold region. Remember that the $S$-wave scattering lengths vanish in the chiral limit and therefore directly measure the symmetry breaking of QCD. This is why these scattering lengths are 
of such fundamental importance. In the modified CHPT, the $\pi \pi$ scattering amplitude reads (up to and including terms of order $q^{3}$ )

$$
A(s, t, u)=\alpha \frac{M_{\pi}^{2}}{3 F_{\pi}^{2}}+\beta \frac{s-4 M_{\pi}^{2} / 3}{F_{\pi}^{2}} .
$$

In the standard scenario, $\alpha=\beta=1$ and one recovers eq.(4.3). In the extreme case that $B$ vanishes in the chiral limit, one has $\alpha=4$ and $\beta=1$. In general, one can interpolate between these two cases via (to leading order)

$$
\alpha^{(2)}=1+6 \frac{\left(2 M_{K}^{2} / M_{\pi}^{2}-1\right)-\left(m_{s} / \hat{m}\right)}{\left(m_{s} / \hat{m}\right)^{2}-1}(1+2 \xi) ; \beta^{(2)}=1,
$$

where $\xi$ accounts for the Zweig rule violation in the $0^{++}$channel and is expected to be small ( $\xi=0$ in what follows). For the value $m_{s} / \hat{m}=10$ advocated in ref.[77], one has $\alpha^{(2)}=1.96$. This leads to $a_{0}^{0}=0.19$ and $a_{0}^{2}=-0.031$ to be compared with the Weinberg result of $a_{0}^{0}=0.16$ and $a_{0}^{2}=-0.045$. The effect is maximal in the combination $a_{0}^{0}+2 a_{0}^{2}[79]$. However, one also has to know the corrections of order $q^{3}$ and $q^{4}$ to these predictions. They have not yet been worked out in detail. The terms of order $q^{3}$ lead to a small increase in $\beta$ and the non-analytic contributions are not known (see below for an estimate). It is, however, clear that this scenario could lead to large value of $a_{0}^{0}$, say $a_{0}^{0}=0.27\left(m_{s} / \hat{m}=6\right.$ at tree level $)$. Such a value could not be accomodated by the standard scenario, cf. eq.(4.8). At present, the data are not accurate enough to differentiate between these two cases. Pion production experiments as recently analyzed by Ochs [73] lead to $a_{0}^{0}=0.23 \pm 0.08$ and the $K_{\ell 4}$ data of Rosselet et al. [80] tied togther with Roy equation constraints lead to $a_{0}^{0}=0.26 \pm 0.05$ [67]. Therefore, a better determination of the $S$-wave scattering lengths is urgently called for. In fact, recent work at Serpuhkov [81] has established the existence of short-lived $\pi^{+} \pi^{-}$atoms. In case they decay from the ground state, their life time is directly proportional to $\left(a_{0}^{0}-a_{0}^{2}\right)^{2}$ [82]. Therefore, a measurement of the life time with an accuracy of 10 per cent would pin down this combination of the $S$-wave scattering lenghts within 5 per cent. A letter of intent for such an experiment at CERN has been presented [83]. In case this experiment is approved and reaches the projected accuracy, it will give strong indications about which value of $B$ is favored. Also, from the $\Phi$ factory DA $\Phi$ E at Frascati one will get very much improved statistics on $K_{\ell 4}$ decays and thus better information on the phase $\delta_{0}^{0}$ in the threshold region.

Finally, we should mention that Crewther [84] has already investigated this problem some time ago. He allowed for a general case $M_{\pi}^{2} \sim \hat{m}^{d}$, with $d$ an integer. $d=1$ gives the standard scenario and $d=2$ the extreme small $B$ case. Taking into account the leading non-analytic terms (chiral logarithms) with a scale of $\mu=800_{-400}^{+800} \mathrm{MeV}$, he compared the predictions for the $\pi \pi$ threshold parameters with the available data. Although neither $d=1$ nor $d=2$ describes the data very well, a slight preference for the standard case is found. This result should only be considered indicative, a complete 
one-loop calculation in the modified CHPT scheme has to be performed so that one has predictions of comparable accuracy to the ones already existing for the standard case (like eq.(4.8)). It is my believe that when the dust settles, the small $B$ alternative will be excluded. Also, the Gell-Mann-Okubo relation for the pseudoscalar masses does not follow naturally in the modified scheme, it requires some parameter fitting. This is a rather unappealling feature. Furthermore, there are indications from lattice gauge calculations supporting the GMOR scenario [85].

\section{3. $\pi K$ scattering}

The scattering process $\pi K \rightarrow \pi K$ is of particular interest because it is the simplest reaction of the Goldstone bosons involving strangeness and unequal meson (quark) masses. Furthermore, since the low energy constants are already determined from other processes, $\pi K$ scattering can serve as a test of the large $N_{c}$ predictions for some of these couplings. It also gives an idea about our understanding of the symmetry breaking in the strange sector. Obviously, corrections to the tree results are expected to be larger than in the two flavor case since the small expansion parameter $\left(M_{\pi} / 4 \pi F_{\pi}\right)^{2}=0.014$ is substituted by $\left(M_{K} / 4 \pi F_{\pi}\right)^{2}=0.18$.

First, some kinematics has to be discussed. In the $s$ channel, there are two independent amplitudes with total isospin $I=1 / 2$ and $I=3 / 2$. The latter is given by the specific process $\pi^{+} K^{+} \rightarrow \pi^{+} K^{+}$, i.e.

$$
T_{\pi K}^{3 / 2}(s, t, u)=T\left(\pi^{+}\left(p_{1}\right) K^{+}\left(p_{2}\right) \rightarrow \pi^{+}\left(p_{3}\right) K^{+}\left(p_{4}\right)\right),
$$

with $s+t+u=2\left(M_{\pi}^{2}+M_{K}^{2}\right)$. Using crossing symmetry, the isospin- $1 / 2$ amplitude follows via $[86]$

$$
T_{\pi K}^{1 / 2}(s, t, u)=\frac{3}{2} T_{\pi K}^{3 / 2}(u, t, s)-\frac{1}{2} T_{\pi K}^{3 / 2}(s, t, u)
$$

The one-loop result for $T_{\pi K}^{3 / 2}(s, t, u)$ has been given in refs. [66,86],

$$
\begin{aligned}
T^{3 / 2}(s, t, u) & =T_{2}(s, t, u)+T_{4}^{T}(s, t, u)+T_{4}^{P}(s, t, u)+T_{4}^{U}(s, t, u) \\
T_{2}(s, t, u) & =\frac{1}{2 F_{\pi}^{2}}\left(M_{\pi}^{2}+M_{K}^{2}-s\right) \\
T_{4}^{T}(s, t, u) & =\frac{1}{16 F_{\pi}^{2}}\left\{\mu_{\pi}\left[10 s-7 M_{\pi}^{2}-13 M_{K}^{2}\right]+\mu_{K}\left[2 M_{\pi}^{2}+6 M_{K}^{2}-4 s\right]\right. \\
& \left.+\mu_{\eta}\left[5 M_{\pi}^{2}+7 M_{K}^{2}-6 s\right]\right\} \\
T_{4}^{P}(s, t, u) & =\frac{2}{F_{\pi}^{2} F_{K}^{2}}\left\{4 L_{1}^{r}\left(t-2 M_{\pi}^{2}\right)\left(t-2 M_{K}^{2}\right)\right. \\
& +2 L_{2}^{r}\left[\left(s-M_{\pi}^{2}-M_{K}^{2}\right)^{2}+\left(u-M_{\pi}^{2}-M_{K}^{2}\right)^{2}\right] \\
& +L_{3}^{r}\left[\left(u-M_{\pi}^{2}-M_{K}^{2}\right)^{2}+\left(t-2 M_{\pi}^{2}\right)\left(t-2 M_{K}^{2}\right)\right] \\
& +4 L_{4}^{r}\left[t\left(M_{\pi}^{2}+M_{K}^{2}\right)-4 M_{\pi}^{2} M_{K}^{2}\right] \\
& \left.+2 L_{5}^{r} M_{\pi}^{2}\left(M_{\pi}^{2}-M_{K}^{2}-s\right)+8\left(2 L_{6}^{r}+L_{8}^{r}\right) M_{\pi}^{2} M_{K}^{2}\right\}
\end{aligned}
$$




$$
\begin{aligned}
T_{4}^{U}(s, t, u)= & \\
& \frac{1}{4 F_{\pi}^{2} F_{K}^{2}}\left\{t(u-s)\left[2 M_{\pi \pi}^{r}(t)+M_{K K}^{2}(t)\right]+\frac{3}{2}\left[( s - t ) \left\{L_{\pi K}(u)+L_{K \eta}(u)\right.\right.\right. \\
& \left.\left.-u\left(M_{\pi K}^{r}(u)+M_{K \eta}^{r}(u)\right)\right\}+\left(M_{K}^{2}-M_{\pi}^{2}\right)\left(M_{\pi K}^{r}(u)+M_{K \eta}^{r}(u)\right)\right] \\
& +\frac{1}{2}\left(M_{K}^{2}-M_{\pi}^{2}\right)\left[K_{\pi K}(u)\left(5 u-2 M_{\pi}^{2}-2 M_{K}^{2}\right)+K_{K \eta}(u)\left(3 u-2 M_{\pi}^{2}-2 M_{K}^{2}\right)\right] \\
& +J_{\pi K}^{r}(s)\left(s-M_{\pi}^{2}-M_{K}^{2}\right)^{2}+\frac{1}{8} J_{\pi K}^{r}(u)\left[11 u^{2}-12 u\left(M_{\pi}^{2}+M_{K}^{2}\right)+4\left(M_{\pi}^{2}+M_{K}^{2}\right)^{2}\right] \\
& +\frac{3}{8} J_{K \eta}^{r}(u)\left(u-\frac{2}{3}\left(M_{\pi}^{2}+M_{K}^{2}\right)\right)^{2}+\frac{1}{2} J_{\pi \pi}^{r}(t) t\left(2 t-M_{\pi}^{2}\right) \\
& \left.+\frac{3}{4} J_{K K}^{r}(t) t^{2}+\frac{1}{2} J_{\eta \eta}^{r}(t) M_{\pi}^{2}\left(t-\frac{8}{9} M_{K}^{2}\right)\right\}
\end{aligned}
$$

The explicit form of the functions $M_{P Q}^{r}$ and $J_{P Q}^{r}$ is given in [14]. The tree level result agrees with the one of Weinberg [62] and Griffith [87]. Notice that the amplitude dependes, like the $\pi \pi$ amplitude (4.4), on the low energy constants $L_{1,2,3,4}^{r}$ but also on $L_{5,6,8}^{r}$.

Fig. 5: Theoretical predictions and empirical data on the $S$-wave scattering lengths. We show the current algebra point ( $C A)$ and the one-loop chiral perturbation theory result (CHPT). The data can be traced back from ref.[66].

In fig.5, the one-loop prediction for the $S$-wave scattering lengths $a_{0}^{1 / 2}$ and $a_{0}^{3 / 2}$ 
is shown in comparison with the data (see ref.[66]), the result of a Roy equation study [88] and a dispersive analysis [89]. The uncertainty in the prediction comes from the uncertainties of the $L_{i}^{r}$ added in quadrature (which is a conservative estimate). It is clear from that figure that a better determination of these scattering lengths would be very much needed. At energies above threshold, the bulk of the data for the phase shift $\delta_{0}^{1 / 2}(s)$ is described fairly well up to $850 \mathrm{MeV}$ (at $800 \mathrm{MeV}$, the one-loop contribution is half as large as the one from the tree level). For the $I=3 / 2$ phase, only few very inaccurate data exist in the threshold region. Similar to the $I=2 \pi \pi S$-wave, the tree and one-loop contributions cancel leading to a strong sensitivity to the values of the low energy constants. In the $P$-wave, the nearby $K^{*}(892)$ resonance forces the one-loop prediction to fail rather quickly. It should be pointed out that the threshold of $\pi K$ scattering is at $633 \mathrm{MeV}$ and therefore the loop corrections can become large rather quickly. It was, however, stressed in ref.[66] that one can continue the amplitudes into the unphysical region and expand around the point $\nu=(s-u) / M_{K}=t=0$. This leads to a satisfactory agreement with existing dispersion-theoretical results [90]. Also, the isospin-even amplitude $T^{(+)}(\nu, t)$ is most sensitive to the actual values of the $L_{i}^{r}$. A better determination of it would allow to test some of the large $N_{c}$ predictions for these couplings. In any case, a more accurate determination of the $\pi K$ scattering amplitudes would serve as a good test of our understanding of the symmetry breaking in the $S U(3)$ sector of QCD.

\subsection{Beyond one loop}

Up to now, we have considered CHPT calculations in the one-loop approximation. In the two flavor case, higher order corrections are generally small at low energies because the expansion parameters are $M_{\pi}^{2} / 16 \pi^{2} F_{\pi}^{2}=0.014$ and $E_{\text {pion }}^{2} / F_{\pi}^{2}$. Furthermore, the amount of work to perform a multi-loop calculation is substantial and no systematic study of the accompanying low energy constants is available. As particularly stressed by Truong and collaborators $[91,92]$ pions in the isospin-zero $S$-state produce strong final state interactions. As a result of this, the one-loop approximation to e.g. the scalar form factor of the pion or to the phase shift $\delta_{0}^{0}$ becomes inaccurate at surprisingly low energies.

The simplest object to study these effects is the scalar form factor (ff) of the pion,

$$
<\pi^{a}\left(p^{\prime}\right) \pi^{b}(p) \text { out }|\hat{m}(\bar{u} u+\bar{d} d)| 0>=\delta^{a b} \tilde{\Gamma}_{\pi}(s)
$$

with $s=\left(p^{\prime}+p\right)^{2}$. It is not directly measurable, but has been determined for energies from threshold to $1 \mathrm{GeV}$ by a dispersive analysis in ref.[40] via solution of the Muskhelishvili-Omnès equations of the $\pi \pi / K \bar{K}$ system (see also [49]). In ref.[93], an explicit calculation of the scalar ff beyond one loop was performed. Consider the normalized scalar ff, $\Gamma_{\pi}(s)=\tilde{\Gamma}_{\pi}(s) / \tilde{\Gamma}_{\pi}(0)$. This quantity is of order one to tree and of order $q^{2}$ at one-loop level. To perform the two-loop calculation, one considers the unitarity relation depicted in fig.6. The key point is that if one has the imaginary part at order 
Fig. 6: Unitarity relation for the scalar form factor of the pion. Only twopion intermediate states contribute to order $q^{4}$, tadpole diagrams are not shown.

$q^{4}$ (to two loops), one can use dispersion relations to get the real part also at two-loop order. To arrive at the imaginary part of the normalized scalar ff to order $q^{4}$, one only has to keep two-pion intermediate states, i.e.

$$
\operatorname{Im} \Gamma_{\pi}=\sigma\left\{T_{0,2}^{0}\left(1+\operatorname{Re} \Gamma_{\pi, 2}\right)+\operatorname{Re} T_{0,4}^{0}\right\} \Theta\left(s-4 m_{\pi}^{2}\right)+\mathcal{O}\left(q^{6}\right),
$$

with $T_{0,2}^{0}$ and $T_{0,4}^{0}$ the $\pi \pi$ scattering amplitude at tree and one-loop level and $\Gamma_{\pi, 2}$ the one-loop expression for the scalar ff (its explicit form can be found in refs.[13,93]). Since these quantities are known from the work of Gasser and Leutwyler [13], Im $\Gamma_{\pi}$ is fixed at order $q^{4}$. The real part can then be evaluated by making use of Cauchy's theorem,

$$
\Gamma_{\pi}(s)=1+\frac{1}{6}<r^{2}>_{S}^{\pi} s+c_{S}^{\pi} s^{2}+\frac{s^{3}}{\pi} \int_{4 M_{\pi}^{2}}^{\infty} \frac{d s^{\prime}}{s^{\prime 3}} \frac{\operatorname{Im} \Gamma_{\pi}\left(s^{\prime}\right)}{s^{\prime}-s-i \epsilon}+\mathcal{O}\left(q^{6}\right),
$$

where the scalar radius of the pion and the curvature $c_{S}^{\pi}$ are given by

$$
\begin{aligned}
<r^{2}>_{S}^{\pi} & =\frac{3}{8 \pi^{2} F_{\pi}^{2}}\left\{\left(\bar{\ell}_{4}-\frac{13}{12}\right)+\frac{\bar{d}_{1} M_{\pi}^{2}}{16 \pi^{2} F_{\pi}^{2}}\right\}+\mathcal{O}\left(M_{\pi}^{4}\right) \\
c_{S}^{\pi} & =\frac{1}{16 \pi^{2} F_{\pi}^{2}}\left\{\frac{19}{120 M_{\pi}^{2}}+\frac{\bar{d}_{2}}{16 \pi^{2} F_{\pi}^{2}}\right\}+\mathcal{O}\left(M_{\pi}^{4}\right) .
\end{aligned}
$$

There appear two unknown parameters, these are the low energy constants $\bar{d}_{1,2}$ related to the effective Lagrangian at two-loop order. They can be fixed by requiring the values of $\left\langle r^{2}>_{S}^{\pi}\right.$ and $c_{S}^{\pi}$ to agree with the ones of the dispersive analysis. This leads to $\bar{d}_{1}=22$ and $\bar{d}_{2}=8.9$. Notice also that the coefficient $\bar{d}_{2}$ contains infrared logarithms so that $\Gamma_{\pi}$ stays finite in the chiral limit.

In fig.7a,b, the real and the imaginary part of the scalar ff to two loops are shown, respectively, in comparison to the one-loop prediction and the empirical curve based on 
Fig. 7: Scalar form factor of the pion. The curves labelled '1', '2', 'O' and ' $B$ ' correspond to the chiral prediction to one-loop, to two-loops, the modified Omnès representation and the result of the dispersive analysis, respectively. The real part is shown in (a) and the imaginary part in (b).

the phase shift analysis of $\mathrm{Au}$, Morgan and Pennington [94]. For the real part, the oneloop approximation reproduces $\operatorname{Re} \Gamma_{\pi}(s)$ within 10 percent for energies below $400 \mathrm{MeV}$ but it fails above $450 \mathrm{MeV}$. In contrast to the bend down of the empirical solution, it rises steadily. The two-loop curve shows the proper behaviour, the broad enhancement

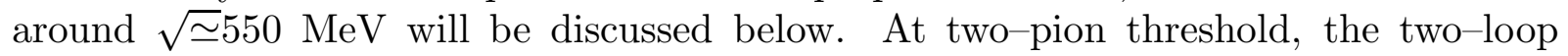
correction to the one-loop enhancement is already substantial. For the imaginary part, the one-loop result fails already very close to threshold. This is not surprising since, quite generally, the imaginary part calculated order by order in the energy expansion, only reflects the real part at one order less. The two-loop prediction is close to the empirical result up to $560 \mathrm{MeV}$. However, since two-loop corrections are large, one might wonder what happens with contributions of yet higher order. I will come back to this point. First, however, a few more comments on the real part of the scalar ff. The reason for the turnover at $\sqrt{s} \simeq 550 \mathrm{MeV}$ can be understood from the fact that one can write $\operatorname{Re} \Gamma_{\pi}$ in an Omnès (exponential) form,

$$
\operatorname{Re} \Gamma_{\pi}(s)=P(s) \mathrm{e}^{\operatorname{Re} \Delta_{0}(s)} \cos \delta_{0}^{0}(s)+\mathcal{O}\left(q^{6}\right),
$$


where the Watson final-state theorem [95] is fulfilled at next-to-leading order, Im $\Delta_{0}(s)=\delta_{0}^{0}(s)+\mathcal{O}\left(q^{6}\right)$. The explicit form of $P(s)$ and $\Delta_{0}(s)$ are given in ref.[93]. Although this representation is not unique, the appearance of the factor $\cos \delta_{0}^{0}$ explains the turnover. The phase $\delta_{0}^{0}$, which enters at one-loop level, goes through $\pi / 2$ at $\sqrt{s} \simeq 680$ $\mathrm{MeV}$ forcing the real part to vanish at this energy. This is clearly a two-loop effect since $\cos \delta_{0}^{0}=1-\left(\delta_{0}^{0}\right)^{2}+\ldots=1+\mathcal{O}\left(s^{2} / F_{\pi}^{4}\right)$ and explains why the one-loop prediction increases monotonically. Furthermore, in ref.[96] the physical interpretation of the broad enhancement in $\operatorname{Re} \Gamma_{\pi}$, which is reminiscent of a resonance structure, was given. Consider the imaginary part (which does not show any resonance behaviour) and subtract from it the uncorrelated two-pion background. This subtracted imaginary part has a bell shape peaked around $600 \mathrm{MeV}$ with a width of $320 \mathrm{MeV}$. The strong final state interactions mock up a broad, low-lying scalar-isoscalar "particle" which is indeed needed to furnish the intermediate-range attraction in the boson-exchange picture of the nucleon-nucleon interaction. The low-lying scalar used in this type of approach is therefore nothing but a convenient representation.

The exponential (Omnès) form (4.23) suggests that higher loops in CHPT can be summed in an easy manner using the final-state theorem. However, since $\Delta_{0}(s)$ behaves badly at high energies and the form (4.23) is not unique, in ref.[93] a modified Omnès form was proposed which cures these shortcomings. It reads

$$
\begin{aligned}
\bar{\Gamma}(s) & =\Gamma^{\Lambda} \mathrm{e}^{\Delta_{\Lambda}(s)}=(1+b \cdot s) \mathrm{e}^{\Delta_{\Lambda}(s)} \\
\Delta_{\Lambda}(s) & =\frac{s}{\pi} \int_{4 M_{\pi}^{2}}^{\infty} \frac{d s^{\prime}}{s^{\prime}} \frac{\Phi\left(s^{\prime}\right)}{s^{\prime}-s-i \epsilon} \\
\tan \Phi & =\frac{\sin 2 \delta_{0}^{0} \mathrm{e}^{-2 \eta}}{1+\cos 2 \delta_{0}^{0} \mathrm{e}^{-2 \eta}}
\end{aligned}
$$

where the cut off $\Lambda$ is chosen around $1 \mathrm{GeV}$ (below $\bar{K} K$ threshold). The reduced Omnès function $\Delta_{\Lambda}(s)$ takes into account the two-pion cut and therefore the reduced $\mathrm{ff} \Gamma^{\Lambda}$ can be well represented by a polynomial linear in $s$ (the slope parameter $b$ is adjusted to the empirical value of the scalar radius). The representation (4.24) is very accurate at low energies. In the chiral limit, $\bar{\Gamma}(s)$ contains the leading and and next-to-leading singularities in $s$. In addition, at each order $N$ in the chiral expansion, the final-state theorem is obeyed,

$$
\bar{\Gamma}_{N}=\mathrm{e}^{2 i \Phi_{N}} \bar{\Gamma}_{N}^{*}
$$

The curves labelled "O" in fig.7a,b show the result of the modified Omnès representation with $\Lambda=1 \mathrm{GeV}$. The real part follows the exact solution closely up to $550 \mathrm{MeV}$. Beyond this energy, it falls off slightly too steeply. However, would one have a more accurate representation of the phase $\Phi$, a better description of the scalar ff would result. The imaginary part reproduces the exact solution within 15 per cent up to $700 \mathrm{MeV}$. The representation (5.7) allows furthermore to investigate the importance of higher loops in the following sense. Expanding $\bar{\Gamma}$ in powers of $q^{2}$ and collecting pieces from the polynom 
and the exponential, one has $\bar{\Gamma}=c_{0}+c_{2}+c_{4}+c_{6}+\ldots$ with $c_{0}=1$ and $c_{2 n}=\mathcal{O}\left(q^{2 n}\right)$. For the real part, one finds that three loop contributions cannot be neglected beyond energies of $500 \mathrm{MeV}$. For the imaginary part the three loop contributions are less than half of the two-loop ones (in magnitude) below $600 \mathrm{MeV}$ and higher loops are negligible. This explains why the already good two-loop result is only mildly affected in this energy range. One can perform the same study for the vector ff of the pion [93]. Below $500 \mathrm{MeV}$, the data have large error bars which makes an exact comparison difficult. However, one finds that the two-loop result describes the data below $500 \mathrm{MeV}$. The polynomial pieces are completely dominant, i.e. the unitarity corrections are small (in contrast to the scalar ff). Clearly, the nearby $\rho$ resonance starts to play a dominant role beyond this energy, indicating that explicit resonance degrees of freedom should be included.

An attempt to incorporate the resonances in EFT was made in ref.[71] in the context of $\pi \pi$ and $\pi K$ scattering. The resonance Lagrangian of Ecker et al. [32] was used and the momentum dependence of the resonance propagators was fully taken into account. This leads to a good description of the data up to energies of $1 \mathrm{GeV}$ and, by construction, the $P$-waves are in perfect agreement with the data. The phase of $\epsilon^{\prime}$ comes out to be $\Phi\left(\epsilon^{\prime}\right)=44.5^{\circ}$, consistent with the value (4.12). However, this calculation is not complete since not all effects at order $q^{6}$ and higher have been taken into account (only the ones related to the various resonance propagators). The whole program of carrying out the loop expansion in an effective theory of pseudoscalar Goldstone bosons coupled to resonances has yet to be performed.

There are some other proposals to enlarge the range of applicability of the truncated chiral expansion. Truong [91] has investigated the scalar and vector ff of the pion. To enforce the final-state theorem to all orders, he considers an Omnès-Muskhelisvhili integral equation for the inverse $\mathrm{ff}$. This is equivalent to summing an infinite series of bubble graphs and formally nothing but the Padé approximant [0,1] (notice that this notation differs from the one used by Truong) of the chiral series. This means that $F=1+F^{(2)}+F^{(4)}$ is substituted by $F_{[0,1]}=F^{(2)} /\left(1-F^{(4)} / F^{(2)}\right)$ with $F$ a generic symbol for a form factor and the subscript gives the chiral dimension. While good agreement with the data is claimed [91], the method was criticized in ref.[93]. First, it was shown that the Padé approximant sums next-to-leading order chiral logarithms with the wrong weight, i.e. it does not respect the constraints from the chiral symmetry. Furthermore, the modified Omnès representation discussed before gives a better description of the scalar ff than the [0,1] approximant (and respects the next-to-leading order logs). Also, the Padé $[0,1]$ apparently describes the data in the resonance region of the $P$-wave well. However, the position, width and heigth of the resonance are very sensitive to the vector radius (which is used to fix the coupling $L_{9}^{r}$ as discussed in section 2.5). Dobado, Herrero and Truong [92] have also applied this method to unitarize the $\pi \pi$ scattering amplitudes. Dobado and Pelaez [97] have recently investigated the inverse amplitude method which is formally equivalent to the Padé $[0,1]$ series. Their philosophy is to fix the couplings $L_{1,2}^{r}$ and $L_{3}$ in some partial waves. Having done that, they find a good description of all phase shifts up to energies of $1 \mathrm{GeV}$ in $\pi \pi$ and $\pi K$ scattering. Notice, 
however, that the data they claim to fit well for the phase $\delta_{0}^{2}(s)$ are outside the Roy equation band discussed before. Clearly, all these unitarization methods are build on the notion of imposing strict (elastic) unitarity on the expense of a controlled chiral expansion. Choosing a particular unitarization procedure induces an unwanted modeldependence and thus violates the strict requirements of CHPT. It is true, however, that higher loop calculations will definitively have to be done by unifying dispersion theoretical methods with chiral low-energy constraints as exemplified in the calculation of the scalar ff discussed before. It is always preferrable to have more loops evaluated than using some unitarization prescription [98].

\subsection{The decays $K \rightarrow 2 \pi$ and $K \rightarrow 3 \pi$}

The non-leptonic weak interactions are a wide field were CHPT methods can be applied, in particular for the many decay modes of the kaons. I will focus here on some recent work in connection with next-to-leading order calculations of the reactions $K \rightarrow 2 \pi$ and $K \rightarrow 3 \pi$ (one reason being that it relates back to the $\pi \pi$ phase shifts discussed before). There are many other articles on kaon decays. Here, let me just mention the nice series of papers by Ecker, Pich and deRafael [99] on decays like $K \rightarrow$ $\pi e^{+} e^{-}, K \rightarrow \pi \mu^{+} \mu^{-}, K^{0} \rightarrow \pi^{0} \gamma \gamma$ and so on. I refer to these papers and the upcoming review by these authors [100] for a comprehensive treatment of these and other $K$-decays (and the list of references therein).

Let us now concentrate on the decays $K \rightarrow 2 \pi, 3 \pi$ at next-to-leading order. To lowest order, the Lagrangian of the strangeness-changing non-leptonic weak interactions reads

$$
\mathcal{L}_{\text {eff }}^{(2)}(\Delta S=1)=-c_{2} \operatorname{Tr}\left(\lambda_{6} U^{\dagger} \partial_{\mu} U U^{\dagger} \partial^{\mu} U\right)-c_{3} t_{i k}^{j l} \operatorname{Tr}\left(Q_{j}^{i} U^{\dagger} \partial_{\mu} U\right) \operatorname{Tr}\left(Q_{l}^{k} U^{\dagger} \partial_{\mu} U\right)
$$

with $\left(Q_{j}^{i}\right)_{k l}=\delta_{i l} \delta_{j k} 3 \times 3$ matrices in flavor space, $\lambda_{6}=Q_{2}^{3}+Q_{3}^{2}$ projects out the octet and the $t_{i k}^{j l}$ the 27-plet of the interactions. This follows from the fact that the chiral transformation properties of the weak interactions are characterized by a decomposition into left-chiral representation as $\left(8_{L}, 1_{R}\right) \oplus\left(27_{L}, 1_{R}\right)$. The so-called weak mass term can be neglected. To lowest order, the constants $c_{2}$ and $c_{3}$ of the $\Delta I=1 / 2$ and $\Delta I=3 / 2$ operators can be fixed from $K \rightarrow 2 \pi$ decays [101],

$$
c_{2} / F_{\pi}^{2}=0.95 \cdot 10^{-7}, c_{3} / F_{\pi}^{2}=-0.008 \cdot 10^{-7}
$$

The fact that $c_{2}>c_{3}$ constitutes the so-called $\Delta I=1 / 2$ rule. At next-to-leading order, the chiral non-leptonic Lagrangian has been worked out by Kambor, Missimer and Wyler [102]. Using chirality and CPS symmetry [103] as the basic principles, one arrives at:

$$
\mathcal{L}_{\mathrm{eff}}^{(4)}(\Delta S=1)=\sum_{i=1}^{47} E_{i}^{r} Q_{i}^{8}+\sum_{j=1}^{33} D_{j}^{r} Q_{j}^{27}
$$


The explicit form of the octet $\left(Q_{i}^{8}\right)$ and the 27-plet $\left(Q_{j}^{27}\right)$ operators can be found in ref.[102]. Not all of these counterterms are independent, but they serve as a useful basis. If one switches off the external fields, one is left with 15 (13) independent contact terms for the octet (27-plet). Clearly, a complete determination of the renormalized weak low-energy constants $E_{i}^{r}$ and $D_{i}^{r}$ is not available (this is one place were the model estimates discussed in section 2.5 are very useful).

Kambor, Missimer and Wyler [104] have considered the decays $K \rightarrow 2 \pi$ and $K \rightarrow$ $3 \pi$ at next-to-leading order. The appearing counterterms can be fitted from decay rates and slope parameters. For doing that, one has to consider the isospin decomposition of the pertinent amplitudes. Consider first the $\mathrm{CP}$-conserving $K \rightarrow 2 \pi$ decays,

$$
\begin{aligned}
A\left(K_{s} \rightarrow \pi^{0} \pi^{0}\right) & =i \sqrt{2 / 3} a_{1 / 2} \mathrm{e}^{i \delta_{0}^{0}}-i \sqrt{4 / 3} a_{3 / 2} \mathrm{e}^{i \delta_{0}^{2}} \\
A\left(K_{s} \rightarrow \pi^{+} \pi^{-}\right) & =-i \sqrt{2 / 3} a_{1 / 2} \mathrm{e}^{i \delta_{0}^{0}}-i \sqrt{4 / 3} a_{3 / 2} \mathrm{e}^{i \delta_{0}^{2}} \\
A\left(K^{+} \rightarrow \pi^{+} \pi^{0}\right) & =-i \sqrt{3 / 4} a_{3 / 2} \mathrm{e}^{i \delta_{0}^{2}}
\end{aligned}
$$

where the coefficients $a_{1 / 2}$ and $a_{3 / 2}$ are real. The two pions in the final state are in an $S$-wave with total isopsin zero or two and therefore the corresponding $\pi \pi$ phases appear (see the discussion after eq.(4.12)). The five $K \rightarrow 3 \pi$ amplitudes are decomposed in terms of two intercepts $\left(\alpha_{1}, \alpha_{3}\right)$, three linear slope $\left(\beta_{1}, \beta_{3}, \gamma_{3}\right)$ and five quadratic slope $\left(\chi_{1}, \chi_{3}, \xi_{1}, \xi_{3}, \xi_{3}^{\prime}\right)$ parameters. These twelve quantities can be expressed in terms of seven combinations of the weak counterterms plus the strong $L_{1, \ldots, 5}^{r}$ together with loop and tree level contributions, the latter involving the parameters $c_{2}, c_{3}, F_{\pi}$ and $F_{K}$ (neglecting terms of order $M_{\pi}^{2} / M_{K}^{2}$ ). In ref.[104] the available data were refitted and then a leastsquare fit was used to determine the values of $c_{2}, c_{3}$ and the $K_{i}(i=1 \ldots, 7)$ from the next-to-leading order expressions. The most prominent result of this analysis is that the value for $c_{2}$ is diminished by 30 per cent,

$$
c_{2} / F_{\pi}^{2}=0.66 \cdot 10^{-7}
$$

to one loop and the 27-plet coupling $c_{3}$ remains unchanged. This reduction of $c_{2}$ implies that a factor 1.5 in the $\Delta I=1 / 2$ enhancement is due to long distance effects (see also refs.[105]).* Notice that the corresponding phase shift difference $\delta_{0}^{0}-\delta_{0}^{2}$ comes out to be $29^{\circ}$, which slightly less than the tree level result of $37^{\circ}$. This is an effect of the fitting procedure since to the order the amplitudes are determined, the imaginary parts should come out at their tree level values. To recover the result (4.12) one would have to go further in the loop expansion. In ref.[104] it was also pointed out that there are two dominating $\Delta I=1 / 2$ counterterms (which have previously been discussed in ref.[107]) which are considerably larger than the two well-determined $\Delta I=3 / 2$ counterterms. This means that the $\Delta I=1 / 2$ enhancement is preserved at next-to-leading order, a

* In ref.[106] it was argued that no suppression of the $\Delta I=3 / 2$ piece due to longdistance effects exists. 
welcome feature. For a more detailed account of these topics, the reader should consult refs. $[102,104]$.

Kambor et al. [108] have further investigated these decay modes. They point

out that to order $M_{\pi}^{2} / M_{K}^{2}$ one can derive relations which are independent of the weak counterterms $K_{1}, \ldots, K_{7}$ at the four derivative level (besides five already known relations at the two derivative level). To be more precise, there are 2 (3) relations in the $\Delta I=1 / 2$ $(3 / 2)$ sector between various of the slope parameters. These conditions follow directly from the CHPT calculation and thus are a highly non-trivial test. The two $\Delta I=1 / 2$ relations are in good agreement with the data where as the comparison in the $\Delta I=3 / 2$ sector is less favorable. However, it is stressed in ref.[108] that large electromagnetic corrections in the $\Delta I=3 / 2$ case are possible and that the analysis in this sector is more sensitive to small errors in the analysis due to cancellation of large numbers. Clearly, a better empirical determination of the decays $K_{L} \rightarrow 3 \pi, K_{L} \rightarrow \pi^{+} \pi^{0} \pi^{-}$and $K^{+} \rightarrow 3 \pi$ would lead to a precision test of CHPT in the $\Delta I=3 / 2$ sector. As a final comment, let me point out that in $\Delta I=1 / 2$ amplitudes one has large loop corrections which are due to the strong pionic final state interactions in the isospin-zero $S$-wave. It is conceivable that a resummation technique as discussed in section 4.4 will allow to sum these rescatterig diagrams in harmony with chiral symmetry.

\section{FINITE TEMPERATURES AND SIZES}

CHPT allows to make precise statements about the low temperature behaviour of the strong interactions. Furthermore, Goldstone boson induced finite size effects can be calculated in a controlled fashion which is of importance for lattice gauge calculations. In this section, I will first discuss some aspects of finite temperatures, mostly the melting of the quark and gluon condensates with increasing temperature. Then, I will cover some developments concerning finite size effects, in particular the comparison of recent Monte Carlo studies of $\sigma$ models and the chiral predictions. Technical details will generally be omitted and the reader should consult the pertinent references.

\subsection{Effective theory at finite temperature}

In the chiral limit, the pions are massless and dominate the spectrum. At sufficiently low temperatures, they do not interact forming a Bose gas with the pressure directly proportional to the fourth power of the temperature. Interactions among the pions generate power-like corrections which are controlled by the parameter $T^{2} / 8 F_{\pi}^{2}$ and the contributions of the heavy particles are exponentially suppressed (see below for details). If the temperature is sufficiently small, a perturbative analysis of these effects can be performed making use of CHPT techniques as developed by Gerber, Gasser and Leutwyler $[109,110,111]$ (see these papers for references on earlier work on this subject). Instead of the $\mathrm{S}$-matrix, one deals with the generating functional $Z$ defined via

$$
Z=\operatorname{Tr}\left[\mathrm{e}^{-H / T}\right]
$$


with $T$ the temperature and $H$ the Hamiltonian. The quantity in the square brackets is nothing but the analytic continuation of the time evolution operator to the point $t=-i / T$. This allows to write

$$
\left(U_{2}\left|\mathrm{e}^{-t H}\right| U_{1}\right)=\int[d U] \exp \left\{-\int d^{4} x \tilde{\mathcal{L}}_{\text {eff }}\right\}
$$

with $U_{1}=U(\vec{x}, 0), U_{2}=U(\vec{x}, t)$ and $\tilde{\mathcal{L}}_{\text {eff }}$ can be obtained from $\mathcal{L}_{\text {eff }}$ by replacing the Minkowski metric $g_{\mu \nu}$ by $-\delta_{\mu \nu}$ and changing the overall sign. To perform the trace, one sets $U_{1}=U_{2}$ and integrates over $U_{1}$ over an interval of length $1 / T$. The functional integral then extends over all field configurations which are periodic in the time direction,

$$
\begin{aligned}
Z & =\int[d U] \exp \left\{-\int_{M} d^{4} x \tilde{\mathcal{L}}_{\mathrm{eff}}\right\} \\
U\left(\vec{x}, x_{4}\right) & =U\left(\vec{x}, x_{4}+\beta\right)
\end{aligned}
$$

with $\beta=1 / T$. The pions live on the manifold $M$ which is nothing but the torus $R^{3} \times S^{1}$. The circumference of $S^{1}$ is given by the inverse temperature $\beta$. It is important to realize that the coupling constants like $F, B, L_{1}^{r}, L_{2}^{r}, \ldots$ remain unaffected (are temperature independent). The boundary conditions in the direction of $x^{4}$ are dictated by the trace that defines the partition function (strictly speaking, the trace operation only makes sense at finite volume. The finite size effects generated by the box are discussed in ref.[112]). The only change induced by the temperature is a modification of the pion propagator. In the chiral limit and in euclidean space, the $T$-dependent propagator is given by

$$
G(x)=\sum_{n=-\infty}^{\infty} \Delta\left(\vec{x}, t+\frac{n}{T}\right)
$$

where $\Delta(x)=\left[4 \pi^{2}\left(\vec{x}^{2}+t^{2}\right)\right]^{-1}$ is the $T=0$ propagator. Any Green function can be evaluated at finite $T$ along the same lines. Denote by $A$ an arbitrary operator (like e.g. the product of two currents), its thermal expectation value is simply given by

$$
<A>_{T}=\frac{\operatorname{Tr}\left[\mathrm{e}^{-\beta H} A\right]}{\operatorname{Tr}\left[\mathrm{e}^{-\beta H}\right]}
$$

In particular, the free energy density $z$ reads

$$
z=\epsilon_{0}-P=-T \lim _{V \rightarrow \infty} \frac{\ln Z}{V}
$$


Fig. 8: Typical two and three loop diagrams for calculating the free energy density. The black dots $(\bullet)$ denote insertions from $\mathcal{L}_{\text {eff }}^{(2)}$.

with $\epsilon_{0}$ the energy density of the vacuum and $P$ the pressure. Clearly, to evaluate the partition function to some given order in the temperature, one has to evaluate CHPT to the corresponding order in the loop expansion. To make this statement more transparent, consider some typical Feynman diagrams contributing to $z$ (fig.8). They have no external legs, however, instead of the small external momenta one now has to deal with temperature insertions. The following remarkable feature emerges [111,113]. Tree diagrams from $\mathcal{L}_{\text {eff }}^{(2,4,6, \ldots)}$ are temperature independent and therefore only contribute to the vacuum energy. If one now evaluates the free energy density to order $q^{n}$, counterterms from $\mathcal{L}_{\text {eff }}^{(n-2)}$ enter through one-loop graphs and thus give rise to a $T$-dependent contribution. This can, however, be absorbed in the renormalization of the pion mass [111]. Therefore, after subtracting the vacuum energy density and expressing the remaining contributions in terms of the physical pion mass, the free energy density evaluated to order $q^{n}$ only involves low-energy constants from the effective Lagrangian up to and including order $n-4$. This makes a three loop calculation feasible since only the known one-loop couplings enter [111]. The calculations are performed in dimensional regularization. In that case, the temperature independent part of the pion propagagtor generates all the singularities as $d \rightarrow 4$ (see ref.[114] for details). Furthermore, most thermodynamic quantities can be expressed in terms of powers of the thermal propagator at $x=0$ and the functions $g_{r}$ associated to the noninteracting $d$-dimensional Bose gas,

$$
g_{r}(M, T)=2 \int_{0}^{\infty} \frac{d \lambda}{(4 \pi \lambda)^{d / 2}} \lambda^{r-1} \exp \left(-\lambda M^{2}\right) \sum_{n=1}^{\infty} \exp \left(-n^{2} / 4 \lambda T^{2}\right) .
$$

This essentially specifies all the tools one needs for finite temperature calculations (for a more detailed account, see ref.[111]).

\subsection{Melting condensates}

The quark condensate is the thermodynamic variable conjugate to the mass of the light quarks. It is given by

$$
<\bar{q} q>_{T}=\frac{\operatorname{Tr}[\bar{q} q \exp (-H / T)]}{\operatorname{Tr}[\exp (-H / T)]}=\frac{\partial z}{\partial m_{q}}
$$


where $m_{q}$ denotes the quark mass. The quark condensate plays the same role as the magnetization in a ferromagnet. Following this analogy, it is supposed to decrease as temperature increases and eventually to disappear. Disorder takes over and the symmetry is restored. In the case of QCD at low temperatures, the pions dominate the properties of the system since the contributions of the massive states to the partition function are exponentially suppressed. The free energy density admits an expansion in powers of $T^{2}$ and $\log T$ much like the $\pi \pi$ scattering amplitude in powers of the external momenta and $\log$ s thereof:

$$
z=\sum_{m, n} c_{m, n}\left(T^{2}\right)^{m}\left(T^{2} \log T\right)^{n}+\mathcal{O}\left(\mathrm{e}^{-\bar{M} / T}\right)
$$

with $m, n=0,1,2, \ldots$ and $\bar{M}$ denotes the mass of the lightest massive state (like the kaon if one works in the two-flavor case). Clearly, eq.(5.9) is an asymptotic series, the exponentially suppressed contributions from the massive states can never be accounted for by powers of $T^{2}$ or logarithms. This is completely analogous to perturbative QCD series, where instantons generate non-perturbative corrections of the type $\exp \left(-8 \pi / g^{2}\right)$. From eq.(5.8) it follows immediately that the thermal expectation value of the quark condensate has a similar representation. For $N_{f}$ massless quarks, the expansion of $<\bar{q} q>_{T}$ reads

$<\bar{q} q>_{T}=<0|\bar{q} q| 0>\left\{1-c_{1}\left(\frac{T^{2}}{8 F_{\pi}^{2}}\right)-c_{2}\left(\frac{T^{2}}{8 F_{\pi}^{2}}\right)^{2}-c_{3}\left(\frac{T^{2}}{8 F_{\pi}^{2}}\right)^{3} \ln \left(\frac{\Lambda_{q}}{T}\right)+\mathcal{O}\left(T^{8}\right)\right\}$

with

$$
c_{1}=\frac{2}{3} \frac{N_{f}^{2}-1}{N_{f}}, c_{2}=\frac{2}{9} \frac{N_{f}^{2}-1}{N_{f}^{2}}, c_{3}=\frac{8}{27}\left(N_{f}^{2}+1\right) N_{f} .
$$

The coefficients $c_{1}, c_{2}$ and $c_{3}$ were first worked out by Binétruy and Gaillard [115], Gasser and Leutwyler [109] and Gerber and Leutwyler [111], respectively. For massless quarks, this result is exact. The first two terms in the expansion are entirely given in terms of the parameter $F_{\pi}$ which characterizes the lowest order effective Lagrangian. Notice also the appearance of the characteristic temperature $T_{c}=\sqrt{8} F_{\pi} \simeq 250 \mathrm{MeV}$. Only at next-to-next-to-leading order a logarithm appears. The unknown scale can be related to the $S U(2)$ low energy constants $\bar{\ell}_{1,2}$ of the next-to-leading order effective Lagrangian. These couplings are related to the isospin-zero $D$-wave scattering length $a_{2}^{0}$ via

$$
a_{2}^{0}=\frac{1}{144 \pi^{3} F_{\pi}^{4}}\left\{\ln \left(\frac{\Lambda_{q}}{M_{\pi}}\right)-0.067+\mathcal{O}(\mathcal{M})\right\}
$$

which leads to $\Lambda_{q}=470 \pm 110 \mathrm{MeV}$ [113]. In figure 9, the one, two and three loop results are shown for temperatures below $150 \mathrm{MeV}$ (using the central value of $\Lambda_{q}$ ). At higher temperatures, the corrections to the tree result become so large that the expansion (5.10) does not make sense any more. In particular, CHPT can not predict 
Fig. 9: The quark condensate at finite temperature (after ref.[111]). The solid, dashed and dashed-dotted line represent the one, two and three loop calculations. The error bar at the three loop curve at $150 \mathrm{MeV}$ represents the uncertainty in the scale of the chiral logarithm.

the temperature of the chiral phase transition, at that point the corrections eat up the leading term completely. The case of non-zero quark masses has also been discussed by Gerber and Leutwyler [111]. As expected, a finite pion mass slows down the melting of the quark condensate. Even though the quark masses are tiny, the temperature dependence is strongly affected because the corresponding Boltzmann factor involves $M_{\pi}$ rather than $M_{\pi}^{2} \sim \hat{m}$. Furthermore, these authors also discuss the influence of the massive states $(K, \eta, \rho, N, \ldots)$ making use of a dilute gas approximation. Below $T=150$ $\mathrm{MeV}$, the massive states influence the melting of the condensate very little because of the Boltzmann factors. Beyond this temperature, one can not neglect the interactions between the massive states and the ones to the pions any more. To summarize, chiral symmetry predicts the temperature dependence of the quark condensate. As the temperature increases, it gradually melts. At a temperature of approximately $150 \mathrm{MeV}$, the loop corrections (in the three loop approximation) have decreased the condensate about a factor of two, rendering the perturbative analysis useless beyond this point. However, if one ignores that for a moment and follows the result to higher temperatures, the condensate vanishes at a temperature of $170 \ldots 190 \mathrm{MeV}$.

In a similar fashion, one can study the temperature dependence of the gluon condensate [116,117] (which is expected to be much weaker than the one of the quark condensate as indicated by some models [118]). For that, one considers the trace of the energy-momentum tensor,

$$
\Theta_{\mu}^{\mu}=-\frac{\beta(g)}{2 g^{3}} G_{\mu \nu}^{a} G^{\mu \nu, a}+\{1+\gamma(g)\} \bar{q} \mathcal{M} q+c \mathbf{1}
$$


Here, $\beta(g)$ is the QCD $\beta$-function, $\mu d g / d \mu=\beta(g)$. The first term in (5.13) accounts for the fact that the strong coupling constant is scale dependent (conformal anomaly) and the second one is due to the explicit scale breaking from the quark masses. The last term is fixed by the normalization condition that the trace of the energy-momentum tensor is zero in the vacuum, i.e. $c=<0\left|G^{2}\right| 0>$, with $G^{2}=-\beta G_{\mu \nu}^{a} G^{\mu \nu, a} / 2 g^{3}$. At finite temperature, one has

$$
<G^{2}>_{T}=<0\left|G^{2}\right| 0>-<\Theta_{\mu}^{\mu}>_{T}
$$

Two comments are in order. First, the gluon condensate is not an order parameter, conformal symmetry is also broken in the high temperature phase of QCD. Second, the vev $<0\left|G^{2}\right| 0>$ can not be determined very accurately, but its value drops out if one considers the temperature dependence of the gluon condensate. The quest is now to calculate $<\Theta_{\mu}^{\mu}>_{T}$. One can make use of the thermodynamic relations $s=d P / d T$ and $\epsilon=T s-P$, with $s$ the entropy density. Therefore, the knowledge of the equation of state $P=P(T)$ is all one needs $[111,112,116]$,

$$
<\Theta_{\mu}^{\mu}>_{T}=\epsilon-3 P=T^{5} \frac{d}{d T}\left(\frac{P}{T^{4}}\right)
$$

Since the correction to the free Bose gas for the massless Goldstone bosons starts out at order $T^{8}$, one finds a weak temperature dependence of the gluon condenstae. For two massless quark flavors, one has [116]

$$
<\Theta_{\mu}^{\mu}>_{T}=\frac{\pi^{2}}{270} \frac{T^{8}}{F_{\pi}^{4}}\left\{\ln \left(\frac{\Lambda_{p}}{T}\right)-\frac{1}{4}\right\}+\ldots
$$

where the ellipsis stands for higher terms in the temperature expansion and the contribution from the exponentially suppressed massive states. One notices that the gluon condensate indeed admits a very weak temperature dependence. The physical reason is that the operators $\Theta_{\mu}^{\mu}$ and $G^{2}$ are chiral singlets and thus single pion emision/absorption is not allowed. Furthermore, $\left\langle\Theta_{\mu}^{\mu}\right\rangle_{T}$ vanishes up to and including two loops in CHPT. This leads to the leading $T^{8}$ term in (5.16). Clearly, since the pion contribution is so much suppressed, the massive states play a more important role already at lower temperatures. For a detailed discussion of these topics, see Gerber and Leutwyler [117].

Finally, one can also study the kinetics of the hot pion gas and its relation to the assumed quark/gluon plasma transition. This topic will not be addressed here, I refer to the article by Goity [119] which also includes references to pertinent work in that field.

\subsection{Effective theory in a box}


One can also address the following question: What happens if the pion fields are confined in a box of finite volume? At first sight, this might sound academical since one knows that there is no spontaneous symmetry breaking in a finite volume. However, CHPT can be used to understand how the infinite volume limit is approached. This is of particular relevance for lattice simulations. On the lattice, the world is a fourdimensional box. In general, one has to deal with finite size effects induced by the particles of mass $M$ which are the lowest excitations in the spectrum. A general analysis of these finite size effects and the approach to the continuum limit has been performed by Lüscher [120] for the case $M L>>1$ ( $L$ denotes the size of the box). Matters are, however, different in the presence of Goldstone bosons (massless excitations). No matter how large one choses the lattice size, the parameter $M L$ will always be small and finite size effects will be large. This can be most easily understood in the case of the quark condensate. For fixed quark masses and zero temperature, the vev $\langle\bar{q} q>$ is always zero as long as $L$ is finite. Only when $L \rightarrow \infty$, symmetry breaking occurs and $\langle\bar{q} q>\neq 0$. Obviously, the finite size effect $<\bar{q} q>_{L}-<\bar{q} q>_{\infty}$ is as big as the quantity itself (see also the discussion by Leutwyler in ref.[116]). As will be shown later on, CHPT can be used to systematically calculate the Goldstone boson induced finite size effects in an expansion in powers of $1 / L^{2}$ (in four dimensions). Ultimately, this will become a tool to control finite size effects in the study of lattice QCD with reasonably light quarks. At present, these methods are tested versus Monte Carlo data on $O(N) \sigma$ models as will be discussed below.

The effective Lagrangian at finite volume $V=L_{1} \times L_{2} \times L_{3} \times L_{4}=L_{1} \times L_{2} \times L_{3} \times \beta$ has been studied in detail by Gasser and Leutwyler [109,112] and also by Neuberger [121]. The lowest order term in the effective theory which generates the leading low energy contributions to the Green functions has the standard form involving the two coupling constants $F$ and $B$. The pion field is subject to periodic boundary conditions,

$$
U(x)=U(x+n), n=\left(n_{1} L_{1}, n_{2} L_{2}, n_{3} L_{3}, n_{4} \beta\right)
$$

with the $n_{i}$ integer numbers. The constants $F$ and $B$ are not affected by the finite volume [112]. As before, the only modification appears in the pion propagator, it takes the form

$$
G(x)=\sum_{n} G_{0}(x+n)
$$

with $G_{0}(x)$ the free $(T=0, L=\infty)$ propagator. The quantities $M_{\pi}, T$ and $1 / L_{i}$ are all booked as $\mathcal{O}(p)$ at fixed ratios $M_{\pi} / T$ and $L_{i} T$ (the so-called " $p$-expansion"). Effectively, one seeks an expansion of the generating functional in powers of these small quantities (a complication arises due to zero modes as discussed below). At next-to-leading order, matters are more complicated. Since the box breaks Lorentz invariance, one expects additional terms related to this phenomenon and also boundary terms which are sensitive to the boundary conditions on the fields. There exists, however, a tremendous simplification if one choses a rectangular box and imposes the same boundary conditions on 
the fields on the walls of the box as in the time direction proportional to $L_{4}$. Then, the partition function is invariant under permutations of the walls of the four-dimensional box. Since we had already seen that the low-energy constants do not depend on the temperature, one can use this symmetry to show that they also do not depend on the finite volume. Furthermore, no Lorentz non-invariant vertices and surface terms appear in this case. Therefore, the effective Lagrangian is just the one used at zero temperature in the infinite volume limit.

Let us now consider $N_{f}$ quarks of the same mass and denote the associated Goldstone boson mass by $M$. The partition function takes the form (for $L_{1}=L_{2}=L_{3}=$ L) $[109]$

$$
\begin{aligned}
& \operatorname{Tr} \mathrm{e}^{-\beta H}=\mathrm{e}^{-\beta L^{3} z} \\
& z=\epsilon_{0}-\frac{N_{f}^{2}-1}{2} g_{0}\left(M^{2}, T, L\right)+\frac{N_{f}^{2}-1}{4 N_{f}} \frac{M^{2}}{F^{2}}\left[g_{1}\left(M^{2}, T, L\right)\right]^{2}+\mathcal{O}\left(p^{8}\right)
\end{aligned}
$$

with $\epsilon_{0}$ the energy density of the groundstate. The functions $g_{0}$ and $g_{1}$ are defined via

$$
g_{r}\left(M^{2}, T, L\right)=\frac{1}{16 \pi^{2}} \int_{0}^{\infty} d \lambda \lambda^{r-3} \sum_{n \neq 0} \exp \left(-M^{2} \lambda-n^{2} / 4 \lambda\right)
$$

These take the familiar form known from the relativistic Bose gas in the infinite volume limit. It is important to omit the term with $n_{1}=n_{2}=n_{3}=n_{4}=0$ from the sum in (5.20). In the chiral limit, the system develops zero modes and consequently the function $g_{1}$ exhibits a pole,

$$
g_{1}\left(M^{2}, T, L\right)=\frac{1}{M^{2} L^{3} \beta}+\bar{g}_{1}\left(M^{2}, T, L\right)
$$

where $\bar{g}_{1}$ is finite when $M$ vanishes. This pole is due to the propagation of the zero modes. Their contribution to the functional integral is not of the Gaussian type as can be seen from

$$
\int d^{4} x \mathcal{L}^{(2)}=-\frac{1}{2} N_{f} F^{2} M^{2} V+\frac{1}{4} F^{2} \int d^{4} x \operatorname{Tr}\left(\partial_{\mu} \phi \partial_{\mu} \phi+M^{2} \phi^{2}\right)+\mathcal{O}\left(p^{4}\right)
$$

The zero modes can therefore not be treated perturbatively. To deal with them, one introduces collective coordinates and reorders the chiral expansion by enhancing the zero-mode propagators (" $\epsilon$-expansion")

$$
T=\mathcal{O}(\epsilon), 1 / L=\mathcal{O}(\epsilon), M=\mathcal{O}\left(\epsilon^{2}\right)
$$

Expanding now the partition function in powers of $\epsilon$, the graphs involving exclusively zero modes are of order one whereas graphs with non-zero modes in the propagators 
are suppressed by at least $\epsilon^{2}$ with the exception of the one-loop graphs which enter in the normalization $A$ of the final result [109]

$$
\operatorname{Tr} \mathrm{e}^{-\beta H}=A X_{N_{f}}(s), s=\frac{1}{2} F^{2} M^{2} V
$$

with $X_{N_{f}}(s)$ the integral over the flavour group

$$
X_{N_{f}}(s)=\int_{S U\left(N_{f}\right)} d \mu(U) \exp (s \operatorname{Re} \operatorname{Tr} U)
$$

and $d \mu(U)$ is the Haar measure. One arrives at this result by the standard procedure introducing collective coordinates, $U=u \mathrm{e}^{i \xi(x)} u$. Here, $\xi(x)$ collects the non-zero modes and the zero modes are described by the constant matrix $u \in S U\left(N_{f}\right)$. For details of this procedure, see Gasser and Leutwyler [109], Hasenfratz and Leutwyler [112] or the original work by Polyakov [123]. In the framework of the $\epsilon$-expansion, one can discuss the symmetry restoration when the quark masses are much smaller than 1 /volume $[109,112,116]$. With this, we have all tools at hand to calculate finite size effects. In the following section, we will compare some theoretical predictions with the results of Monte Carlo studies.

\subsection{Finite size effects: CHPT versus Monte Carlo}

Here I will briefly discuss the comparison of theoretical predictions of Goldstone boson induced finite size effects with recent numerical simulations. For all technical details, I refer the reader to the pertinent articles. First, consider models with broken $O(N)$ symmetries in more than two dimensions. These are of relevance in the study of the Higgs mass bounds, two-flavour QCD or finite size effects in ferromagnets close to the critical point. Hasenfratz and Leutwyler [112] have given theoretical predictions for the free energy, magnetization, susceptibilities and two-point functions up to and including order $\left(1 / L^{d-2}\right)^{2}$ corrections for $d=3$ and $d=4$. In the first case, only two low-energy constants enter whereas in four dimensions, one has to deal with three additional ones. They use the linear $\sigma$-model in $d$ dimensions making use of "magnetic" language,

$$
\mathcal{L}=\frac{1}{2} \partial_{\mu} \phi^{a} \partial_{\mu} \phi^{a}+\frac{1}{2} m^{2} \phi^{a} \phi^{a}+\frac{1}{4} \lambda\left(\phi^{a} \phi^{a}\right)^{2}-H \phi^{0}
$$

The real field $\phi$ has $N(a=0, \ldots, N-1)$ components and the external magentic field $H=(H, 0, \ldots, 0)$ breaks the $O(N)$ symmetry explicitely. As $H$ tends to zero, we are interested in the coupling constant regime where spontaneous symmetry breaking $O(N) \rightarrow O(N-1)$ occurs signaled by a non-vanishing value of the magnetisation $\Sigma$,

$$
\lim _{H \rightarrow 0}<\phi^{0}>=\Sigma,<\phi^{i}>=0
$$


Translated to QCD language, $\Sigma$ is the vev of the scalar quark density and $H$ is equivalent to the quark mass matrix. The corresponding effective theory is formulated in terms of a vector field $S^{a}(x)$ which embodies the $N-1$ Goldstone fields and is subject to the constraint $S^{a}(x) S^{a}(x)=1$. The low energy properties of this model and the finite size effects at next-to-leading order are discussed in detail in ref.[122]. In three dimension, there are two low energy constants entering and in $d=4$ there are five, the extra three being related to scales of logarithmic corrections.

For the $d=3$ classical $O(3)$ Heisenberg model in the broken phase near the critical point, some of these predictions were confronted with Monte Carlo data by Dimitrovic et al. [124]. They found that the finite size behaviour of the magnetisation and the correlation functions are in good agreemnet with the CHPT predictions. Furthermore, they could determine the critical indices for the correlation length and magnetisation (in agreement to previous studies using other methods). In the case of the $O(4)$ model in four dimensions, numerical studies have been performed by Hasenfratz et al. [125]. Good agreement with the theoretical predictions is found and it is shown that in situations where the Goldstone bosons control the dynamics of the system, one can indeed determine the infinite volume, zero external source quantities from the finite volume simulations in a controlled way. Another quantity of interest is the so-called constrained effective potential which in the context of the non-linear $\sigma$-model has been studied by Göckeler and Leutwyler [126]. A numerical simulation has been presented by Dimitrovic et al. [127]. They found good agreement for the shape of the constrained effective potential in the vicinity of its minimum, but also noted that this method is not as accurate in determining the low energy constants $\Sigma$ and $F$ than the one presented in [125].

Hansen [128] has extended this analysis to theories with $S U(N) \times S U(N)$ broken symmetries and Hansen and Leutwyler [129] have studied the charge correlations and topological susceptibility of QCD at finite volume and temperature. It is conceivable that these predictions will become useful when one will be able to perform QCD simulations with dynamical fermions of reasonably small masses. At present, many Monte Carlo studies are done in the quenched approximation (i.e. suppressing fermion loops). In that case, the theoretical predictions are not applicable. Effective theories in the quenched approximation are being developed by Sharpe [130] and Bernard and Golterman [131]. Finally, Leutwyler and Smilga [132] have discussed the spectrum of the Dirac operator and the role of the winding number in QCD. The distribution of winding number and the spectrum of the Dirac operator at small eigenvalues are related to the quark condensate at infinite volume, which implies that the formation of the quark condensate is connected to the occurrence of eigenvalues of the order $\lambda_{n} \sim 1 / V$. What their analysis can not provide is a reason why a condensate arises. For a more detailed discussion of these and other topics, the reader should consult ref.[132].

\subsection{An application to high- $T_{c}$ superconductivity}


To demonstrate the universality of the CHPT methods, let me briefly discuss some work by Hasenfratz and Niedermayer [133] on the correlation length of the antiferromagnetic $(\mathrm{AF}) d=2+1$ Heisenberg model at low temperatures. One of their motivations is the recent discovery of quasi-two-dimensional anti-ferromagnets like the undoped $\mathrm{La}_{2} \mathrm{CuO}_{4}$ compound. In this crystal, a single unpaired electron at each $\mathrm{Cu}^{++}$ site makes the material antiferromagnetic with a quasi-two-dimensional structure. This means that the forces in the plane are much stronger than the forces between neighbouring planes. As it is well known, in doped $\mathrm{La}_{2} \mathrm{CuO} \mathrm{O}_{4}$ one observe high $T_{c}$ superconductivity. Doping e.g. with $S r$ atoms renders the planes metallic and leads to the superconductivity. To understand this phenomenon one has to understand first the physics of the undoped $\mathrm{La}_{2} \mathrm{CuO}_{4}$ system. Due to the particular structure of this crystal, one can use CHPT methods to understand its low temperature behaviour. It should be well described by the $d=d_{s}+1=2+1$ dimensional quantum Heisenberg model,

$$
\mathcal{H}=J \sum_{<i j>} \vec{S}_{i} \vec{S}_{j} \quad(J>0)
$$

where $\vec{S}_{i}$ is the spin operator at site $i$ and $\vec{S}_{i}^{2}=S(S+1)$. In the case of $L a_{2} C u O_{4}$, one has $S=1 / 2$. The groundstate is assumed to be antiferromagnetically ordered (which is strictly proven only for $|S| \geq 1$ ). In the case of a non-vanishing value of the coupling strength $J$, the rotational symmetry is broken and one has two (almost) massless Goldstone bosons (magnons). These modes pick up a small mass since the MerminWagner-Coleman theorem [134] forbids massless Goldstone bosons in two dimensions at finite temperature. These magnons interact weakly at low temperatures, $T<<J$. This makes an analytic study feasible. Note, however, that at very low energies (much smaller than $T$ ) the magnon interaction starts to become stronger again which leads to a finite mass gap in the theory. The exact result for the mass gap of the $d=2 \mathrm{O}(3)$ non-linear $\sigma$-model has been given in ref.[135] and can also be used in the present case. The temperature dependence of the correlation length $\xi$ can now be calculated from an effective Lagrangian which shares the properties of the quantum AF model (5.28). The details are given in ref.[133] and the final result reads:

$$
\xi(T)=\frac{e}{8} \frac{\hbar c}{2 \pi F^{2}} \exp \left(2 \pi F^{2} / T\right)\left[1-\frac{1}{2} \frac{T}{2 \pi F^{2}}+\mathcal{O}\left(\left(\frac{T}{2 \pi F^{2}}\right)^{2}\right)\right]
$$

with $F^{2}$ the spin-stiffness and $c$ the spin-velocity (notice that in $d=3, F$ has dimension [energy $]^{1 / 2}$ ). The result (5.29) applies for the temperature range $T_{N}<T<<2 \pi F^{2}$, with $T_{N} \simeq 300 \mathrm{~K}$ the temperature until which the $\mathrm{La}_{2} \mathrm{CuO}_{4}$ compound remains in the broken phase. A recent measurement of the spin-velocity gives $\hbar c=(850 \pm 30) \mathrm{meV} \AA$ [136]. Notice that the temperature dependence of $\xi(T)$ is essentially given by the exponential prefactor (which was already predicted in ref.[137]), at $600 \mathrm{~K}$ the correction $T / 4 \pi F^{2}$ is $\sim 17$ percent. The important prefactor $e / 8$ comes from the exact form of the mass 
Fig. 10: Inverse correlation length of undoped $\mathrm{La}_{2} \mathrm{CuO}_{4}$. The solid line is the CHPT prediction, eq.(5.29), and the dashed one accounts only for the first term in the square brackets. The data are taken from refs.[138,139].

gap. In fig.10, a one-parameter fit to the recent data is shown, one finds for the spinstiffness $2 \pi F^{2}=150 \mathrm{meV}=1740 \AA$ [138]. Clearly, the fit is not perfect, but this can not be expected. Anisotropies in the spin-spin interactions might lead to such deviations. Phenomenologically, it was found that for doped samples the following rule holds: $\xi^{-1}(x, T)=\xi^{-1}(x, 0)+\xi^{-1}(0, T)$ with $x$ the doping concentration and the formula (5.29) applies to $x=0$. For a further discussions, see refs.[137] and the talk by Hasenfratz [139]. Wiese and Ying [140] have recently performed a Monte Carlo simulation of the 2-d antiferromagnetic quantum Heisenberg model. They compare the finite size and temperature effects with the ones given by the chiral perturbation theory calculation of Hasenfratz and Niedermayer [140]. They find $\hbar c=(845 \pm 32) \mathrm{meV} \AA$ and $2 \pi F^{2}=(157 \pm 7) \mathrm{meV}$, in good agreement with the data and the one-parameter fit described above. They also show results for the ground state energy density and the staggered magnetisation. This again demonstrates the usefulness of the EFT approach. 


\section{BARYONS}

\subsection{Relativistic formalism}

In this section, I will be concerned with the inclusion of baryons in the effective field theory. The relativistic formalism dates back in the early days, see e.g. Weinberg [10], Callan et al. [11], Langacker and Pagels [14] and others (for reviews, see Pagels [8] and the book by Adler and Dashen [4]). The connection to QCD Green functions was performed in a systematic fashion by Gasser, Sainio and Švarc [142] (from here on referred as GSS) and Krause [143]. As done in the GSS paper, I will outline the formalism in the two-flavor case, i.e. for the pion-nucleon $(\pi N)$ system. The extension to full flavor $S U(3)$ is spelled out in appendix B.

There is a variety of ways to describe the transformation properties of the spin- $-1 / 2$ baryons under chiral $S U(2) \times S U(2)$. All of them lead to the same physics. However, there is one most convenient choice (this is discussed in detail in Georgi's book [21]). Combine the proton $(p)$ and the neutron $(n)$ fields in an isospinor $\Psi$

$$
\Psi=\left(\begin{array}{l}
p \\
n
\end{array}\right)
$$

The Goldstone bosons are collected in the matrix-valued field $U(x)$. In the previous sections, we had already seen that the self-interactions of the pions are of derivative nature, i.e. they vanish at zero momentum transfer. This is a feature we also want to keep for the pion-baryon interaction. It calls for a non-linear realization of the chiral symmetry. Following Weinberg [10] and Callan et al. [11], we introduce a matrixvalued function $K$. It not only depends on the group elements $L, R \in S U(2)_{L, R}$, but also on the pion field (parametrized in terms of $U(x)$ ) in a highly non-linear fashion, $K=K(L, R, U)$. Since $U(x)$ depends on the space-time coordinate $x, K$ implicitely depends on $x$ and therefore the transformations related to $K$ are local. To be more specific, $K$ is defined via

$$
L u=u^{\prime} K
$$

with $u^{2}(x)=U(x)$ and $U^{\prime}(x)=R U(x) L^{\dagger}=u^{\prime 2}(x)$. The transformation properties of the pion field induce a well-defined transformation of $u(x)$ under $S U(2) \times S U(2)$. This defines $K$ as a non-linear function of $L, R$ and $\pi(x) . K$ is a realization of $S U(2) \times S U(2)$,

$$
K=\sqrt{L U^{\dagger} R^{\dagger}} R \sqrt{U}
$$

and the baryon field transforms as

$$
\Psi \rightarrow K(L, R, U) \Psi
$$

The somewhat messy object $K$ can be understood most easily in terms of infinitesimal transformations. For $K=\exp \left(i \gamma_{a} \pi_{a}\right), L=\exp \left(i \alpha_{a} \pi_{a}\right) \exp \left(i \beta_{a} \pi_{a}\right)$ and $R=$ $\exp \left(-i \alpha_{a} \pi_{a}\right) \exp \left(i \beta_{a} \pi_{a}\right)$ (with $\gamma_{a}, \alpha_{a}, \beta_{a}$ real) one finds,

$$
\vec{\gamma}=\vec{\beta}+i[\vec{\alpha}, \vec{\pi}] / F_{\pi}+\mathcal{O}\left(\vec{\alpha}^{2}, \vec{\beta}^{2}, \vec{\pi}^{2}\right)
$$


which means that the nucleon field is multiplied with a function of the pion field. This gives some credit to the notion that chiral transformations are related to the absorption or emission of pions. To construct now the lowest order effective Lagrangian of the pion-nucleon system, one has to assemble all building blocks. These are the mesons and baryon fields, $U(x)$ and $\Psi(x)$, respectively as well as the appropriate covariant derivatives (I restrict myself here to the case with only scalar and vector external fields. The more general expressions are given in Appendix B),

$$
\begin{aligned}
\nabla_{\mu} U & =\partial_{\mu} U-i e \mathcal{A}_{\mu}[Q, U] \\
D_{\mu} \Psi & =\partial_{\mu} \Psi+\Gamma_{\mu} \Psi \\
\Gamma_{\mu} & =\frac{1}{2}\left\{u^{\dagger}\left(\partial_{\mu}-i e \mathcal{A}_{\mu} Q\right) u+u\left(\partial_{\mu}-i e \mathcal{A}_{\mu} Q\right) u^{\dagger}\right\}
\end{aligned}
$$

with $Q=\operatorname{diag}(1,0)$ the nucleon charge matrix and $v_{\mu}=e\left(1+\tau_{3}\right) \mathcal{A}_{\mu} / 2$ the external vector field $\left(\mathcal{A}_{\mu}\right.$ denotes the photon field). $D_{\mu}$ transforms homogeneously under chiral transformations, $D_{\mu}^{\prime}=K D_{\mu} K^{\dagger}$. The object $\Gamma_{\mu}$ is the so-called chiral connection. It is a gauge field for the local transformations

$$
\Gamma_{\mu}^{\prime}=K \Gamma_{\mu} K^{\dagger}+K \partial_{\mu} K^{\dagger}
$$

The connection $\Gamma_{\mu}$ contains one derivative. One can also form an object of axial-vector type with one derivative,

$$
\frac{1}{2} u_{\mu}=\frac{i}{2}\left(u^{\dagger} \nabla_{\mu} u-u \nabla_{\mu} u^{\dagger}\right)=\frac{i}{2}\left\{u^{\dagger}, \nabla_{\mu} u\right\}=i u^{\dagger} \nabla_{\mu} U u^{\dagger}
$$

which transforms homogeneously, $u_{\mu}^{\prime}=K u_{\mu} K^{\dagger}$. The covariant derivative $D_{\mu}$ and the axial-vector object $u_{\mu}$ are the basic building blocks for the lowest order effective theory. Before writing it down, let us take a look at its most general form. It can be written as a string of terms with an even number of external nucleons, $n_{\text {ext }}=0,2,4, \ldots$. The term with $n_{\text {ext }}=0$ obviously corresponds to the meson Lagrangian (2.9) so that

$$
\mathcal{L}_{e f f}[\pi, \Psi, \bar{\Psi}]=\mathcal{L}_{\pi \pi}+\mathcal{L}_{\bar{\Psi} \Psi}+\mathcal{L}_{\bar{\Psi} \Psi \bar{\Psi} \Psi}+\ldots
$$

Typical processes related to these terms are pion-pion, pion-nucleon and nucleonnucleon scattering, in order. In what follows, we will mostly be concerned with processes with two external nucleons,

$$
\mathcal{L}_{\bar{\Psi} \Psi}=\mathcal{L}_{\pi N}=\bar{\Psi}(x) \mathcal{D}(x) \Psi(x)
$$

The differential operator $\mathcal{D}(x)$ is now subject to a chiral expansion. Its explicit form to lowest order follows simply by combining the connection $\Gamma_{\mu}$ and the axial-vector $u_{\mu}$ 
(which are the objects with the least number of derivatives) with the appropriate baryon bilinears

$$
\begin{aligned}
\mathcal{L}_{\pi N}^{(1)} & =\bar{\Psi} \mathcal{D}^{(1)} \Psi \\
& =\bar{\Psi}\left(i \gamma_{\mu} D^{\mu}-\stackrel{\circ}{m}+\frac{i}{2} \stackrel{\circ}{g}_{A} \gamma^{\mu} \gamma_{5} u_{\mu}\right) \Psi
\end{aligned}
$$

The effective Lagrangian (6.11) contains two new parameters. These are the baryon mass $\stackrel{\circ}{m}$ and the axial-vector coupling $\stackrel{\circ}{g}_{A}$ in the chiral limit,

$$
\begin{aligned}
m & =\stackrel{\circ}{m}[1+\mathcal{O}(\hat{m})] \\
g_{A} & =\stackrel{\circ}{g}_{A}[1+\mathcal{O}(\hat{m})]
\end{aligned}
$$

Here, $m=939 \mathrm{MeV}$ denotes the physical nucleon mass and $g_{A}$ the axial-vector strength measured in neutron $\beta$-decay, $n \rightarrow p e^{+} \bar{\nu}_{e}, g_{A} \simeq 1.26$. The fact that $\stackrel{m}{m}$ does not vanish in the chiral limit (or is not small on the typical scale $\Lambda \simeq M_{\rho}$ ) will be discussed below. Furthermore, the actual value of $\stackrel{m}{m}$, which has been subject to much recent debate, will be discussed in the context of pion-nucleon scattering. The occurence of the constant $\stackrel{\circ}{g}_{A}$ is all but surprising. Whereas the vectorial (flavor) $S U(2)$ is protected at zero momentum transfer, the axial current is, of course, renormalized. Together with the Lagrangian $\mathcal{L}_{\pi \pi}^{(2)}(2.9)$, our lowest order pion-nucleon Lagrangian reads:

$$
\mathcal{L}_{1}=\mathcal{L}_{\pi N}^{(1)}+\mathcal{L}_{\pi \pi}^{(2)}
$$

To understand the low-energy dimension of $\mathcal{L}_{\pi N}^{(1)}$, we have to extend the chiral counting rules of section 2.3 to the various operators and bilinears involving the baryon fields. These are:

$$
\begin{aligned}
& \stackrel{\circ}{m}=\mathcal{O}(1), \Psi, \bar{\Psi}=\mathcal{O}(1), D_{\mu} \Psi=\mathcal{O}(1) \\
& \bar{\Psi} \gamma^{\mu} \gamma_{5} \Psi=\mathcal{O}(1), \bar{\Psi} \sigma^{\mu \nu} \Psi=\mathcal{O}(1), \bar{\Psi} \sigma^{\mu \nu} \gamma_{5} \Psi=\mathcal{O}(1) \\
& (i \not D-\stackrel{\circ}{m}) \Psi=\mathcal{O}(p), \quad \bar{\Psi} \gamma_{5} \Psi=\mathcal{O}(p)
\end{aligned}
$$

Here, $p$ denotes a generic nucleon three-momentum. Since $\stackrel{\circ}{m}$ is of order one, baryon four-momenta can never be small on the typical chiral scale. Stated differently, any time derivative $D_{0}$ acting on the spin-1/2 fields brings down a factor $\stackrel{m}{m}$. However, the operator $(i \not D-\stackrel{\circ}{m}) \Psi$ counts as order $\mathcal{O}(p)$. The proof of this can be found in ref.[143] or in the lectures [144].

To exhibit the physics content of the Lagrangian $\mathcal{L}_{\pi N}^{(1)}(6.11)$, let me parametrize the pions in the " $\sigma$-model" gauge (which is a convenient choice),

$$
U=(\sigma+i \pi) / F, \quad \sigma^{2}+\pi^{2}=F^{2}
$$

and expand the connection $\Gamma_{\mu}$ as well as the axial-vector $u_{\mu}$ in powers of the pion fields:

$$
\begin{aligned}
i \Gamma_{\mu} & =v_{\mu}+\frac{i}{8 F^{2}}\left[\pi, \partial_{\mu} \pi\right]-\frac{1}{8 F^{2}}\left[\pi,\left[\pi, v_{\mu}\right]\right]+\ldots \\
\frac{1}{2} u_{\mu} & =-\frac{1}{2 F} \partial_{\mu} \pi+\frac{i}{2 F}\left[v_{\mu}, \pi\right]-\frac{1}{16 F^{3}}\left\{\pi,\left\{\pi, \partial_{\mu} \pi\right\}\right\}+\ldots
\end{aligned}
$$


Fig. 11: Feynman rules in the $\sigma$-model gauge. Nucleons, pions and photons are denoted by solid, dashed and wiggly lines, respectively. 
The corresponding vertices are shown in fig.11. Clearly, one recovers some wellknown vertices like the pseudovector (derivative) $\pi N$ coupling or the Kroll-Ruderman term which plays an important role in pion photoproduction. Notice that all prefactors are given in terms of the four lowest-order constants, $F, B, \stackrel{\circ}{g}_{A}$ and $\stackrel{\circ}{m}$. To lowest order, the Goldberger-Treiman relation [145] is exact, $\stackrel{\circ}{g}_{A} \stackrel{\circ}{m}=\stackrel{\circ}{g}_{\pi N} F$, which allows us to write the $\pi N$ coupling in the more familiar form $\sim \stackrel{\circ}{g}_{\pi_{N}} \partial_{\mu} \pi^{a}$.

It goes without saying that we have to include pion loops, associated with $\mathcal{L}_{1}$ given in (6.13). The corresponding generating functional reads [142]

$$
\begin{aligned}
\exp \{i \mathcal{Z}\} & =N \int[d U] \exp \left\{i \int d x \mathcal{L}_{\pi \pi}^{(2)}+i \int d x \bar{\eta} S^{(1)} \eta\right\} \\
\mathcal{D}^{(1) a c} S^{(1) c b} & =\delta^{a b} \delta^{(4)}(x-y)
\end{aligned}
$$

with $\eta, \bar{\eta}$ external Grassmannian sources and $S^{(1)}$ the inverse nucleon propagator related to $\mathcal{D}^{(1)}$ (6.11). $a, b$ and $c$ are isospin indices. This generating functional can now be treated by standard methods. The details are spelled out by GSS [142]. I will now concentrate on the low-energy structure of the effective theory which emerges. Pion loops generate divergences, so one has to add counterterms. This amounts to

$$
\begin{aligned}
& \mathcal{L}_{1} \rightarrow \mathcal{L}_{1}+\mathcal{L}_{2} \\
& \mathcal{L}_{2}=\Delta \mathcal{L}_{\pi N}^{(0)}+\Delta \mathcal{L}_{\pi N}^{(1)}+\mathcal{L}_{\pi N}^{(2)}+\mathcal{L}_{\pi N}^{(3)}+\mathcal{L}_{\pi \pi}^{(4)}
\end{aligned}
$$

Let me first discuss the last three terms on the r.h.s. of eq.(6.18). These are the expected ones. The structure of the $\pi N$ interaction allows for odd powers in $p$, so starting from $\mathcal{L}_{\pi N}^{(1)}$ to one-loop order one expects couterterms of dimension $p^{2}$ and $p^{3}$. A systematic analysis of all these terms has been given by Krause [143]. The first two terms, $\Delta \mathcal{L}_{\pi N}^{(0)}$ and $\Delta \mathcal{L}_{\pi N}^{(1)}$, are due to the fact that the lowest order coefficients $\stackrel{\circ}{m}$ and $\stackrel{\circ}{g}_{A}$ are renormalized (by an infinite amount) when loops are considered. This is completely different from the meson sector, the constants $B$ and $F$ are not renormalized by the loops. The origin of this complication lies in the fact that the nucleon mass does not vanish in the chiral limit. To avoid any shift in the values of $\stackrel{\circ}{m}$ and $\stackrel{\circ}{g}_{A}$ one thus has to add

$$
\Delta \mathcal{L}_{\pi N}^{(0)}=\Delta \stackrel{\circ}{m}\left(\frac{\stackrel{\circ}{m}}{F}\right)^{2} \bar{\Psi} \Psi, \Delta \mathcal{L}_{\pi N}^{(1)}=\Delta \stackrel{\circ}{g}_{A}\left(\frac{\stackrel{\circ}{m}}{F}\right)^{2} \frac{1}{2} \bar{\Psi} i \gamma^{\mu} \gamma_{5} u_{\mu} \Psi
$$

The first term in (6.19) can be easily worked out when one considers the nucleon self-energy $\Sigma_{N}(p)$ related to the nucleon propagator via $S(p)=\left[\stackrel{\circ}{m}-\not p-\Sigma_{N}(p)\right]^{-1}$ in the one-loop approximation [142]. The low-energy structure of the theory in the presence of baryons is much more complicated than in the meson sector. This becomes most transparent when one compares the $\pi \pi$ and $\pi N$ scattering amplitudes, $T_{\pi \pi}$ and $T_{\pi N}$, respectively. While $T_{\pi \pi}^{\text {tree }} \sim p^{2}$ and $T_{\pi \pi}^{\mathrm{n}-\text { loop }} \sim\left(p^{2}\right)^{n+1}$, the corresponding behaviour for $T_{\pi N}$ is shown in fig.12 [142]. Here, $p$ denotes either a small meson four-momentum or 
Fig. 12: Chiral expansion for the $\pi N$ scattering amplitude, $T_{\pi N} \sim p^{N}$. Tree graphs contribute at $N=1,2,3 \ldots$ n-loop graphs at $N=2,3, \ldots$ (after mass and coupling constant renormalization). The contributions from 2,3,... loops are analytic in the external momenta at order $p^{3}$ (here, $p$ is a pion four-, nucleon three-momnentum or the pion mass).

mass or a nucleon three-momentum. Tree graphs for $T_{\pi N}$ start out at order $p$ followed by a string of higher order corrections $p^{2}, p^{3}, \ldots$ One-loop graphs start out at order $p^{2}$ (after appropriate mass and coupling constant renormalization) and are non-analytic in the external momenta at order $p^{3}$ (in the chiral limit $\hat{m}=0$ ). Higher loops start out at $p^{2}$ and are analytic to orders $\mathcal{O}\left(p^{2}, p^{3}\right)$. This again means that the low-energy constants associated to $\mathcal{L}_{\pi N}^{(2,3)}$ will get renormalized. Evaluation of one-loop graphs associated with $\mathcal{L}_{1}$ therefore produces all non-analytic terms in the external momenta of order $p^{3}$ like e.g. leading threshold or branch point singularities. Let us now consider the case $\hat{m} \neq 0$. Obviously, the $\pi N$ amplitude also contains terms which are nonanalytic in the quark masses. A good example is the Adler-Weisberger relation in its differential form - it contains a factor $F_{\pi}^{-2}$ and therefore a term which goes like $\hat{m} \ln \hat{m}$. Due to the complicated low-energy structure of the meson-baryon system, it has never been strictly proven that one-loop graphs generate all leading infrared singularities, in particular the ones in the quark masses. However, in all calculations performed so far the opposite has never been observed. In any case, the exact one-to-one correspondence between the loop and small momentum expansion is not valid in the meson-baryon system if one treats the baryons fully relativistically. This can be overcome, as will be discussed in the next section, in an extreme non-relativistic limit. Here, however, I wish to point out that the relativistic formalism has its own advantages. Two of them are the direct relation to dispersion theory and the inclusion of the proper relativistic kinematics in certain processes. These topics will be discussed later on. Let me make a few remarks concerning the structure of the nucleons (baryons) at low energies. Starting 
from a structureless Dirac field, the nucleon is surrounded by a cloud of pions which generate e.g. its anomalous magnetic moment (notice that the lowest order effective Lagrangian (6.11) does only contain the coupling of the photon to the charge). Besides the pion loops, there are also counterterms which encode the traces of meson and baryon excitations contributing to certain properties of the nucleon. Finally, one point which should be very clear by now: One can only make a firm statement in any calculation if one takes into account all terms at a given order. For a one-loop calculation in the meson-baryon system, this amounts to the tree terms of order $p$, the loop contributions of order $p^{2}, p^{3}$ and the counterterms of order $p^{2}$ and $p^{3}$. This should be kept in mind in what follows.

\subsection{Non-relativistic formalism}

As we saw, the fully relativistic treatment of the baryons leads to severe complications in the low-energy structure of the EFT. Intuitively, it is obvious how one can restore the one-to-one correspondence between the loop and the small momentum expansion. If one considers the baryons as extremely heavy, only the relative pion momenta will count and these can be small. The emerging picture is that of a very heavy source surrounded by a cloud of light (almost massless) particles. This is exactly the same idea which is used in the so-called heavy quark effective field theory methods used in heavy quark physics. To be precise, consider a meson of the type $Q \bar{q}$ (or $\bar{Q} q$ ), with $Q=b, t$ the heavy and $q=u, d$ the light quark. The light quarks and the gluons are a cloud around the heavy source $Q$, they form the so-called "brown mug". This is the picture underlying the so-called heavy quark symmetries in QCD [146] which allows e.g. to relate a set of form factors to one invariant function (sometimes called the "Isgur-Wise function"). Therefore, it appears natural to apply the insight gained from heavy quark EFT's to the pion-nucleon sector. Jenkins and Manohar $[147,148,149]$ have given a new formulation of baryon CHPT based on these ideas. It amounts to taking the extreme non-relativistic limit of the fully relativistic theory and an expansion in powers of the inverse baryon mass. Obviously, the relativistic theory and its extreme non-relativistic limit are connected by a series of Foldy-Wouthuysen transformations [150].

Let me first spell out the underlying ideas before I come back to the $\pi N$ system. Our starting point is a free Dirac field with mass $m$

$$
\mathcal{L}=\bar{\Psi}(i \not \partial-m) \Psi
$$

Consider the spin-1/2 particle very heavy. This allows to write its four-momentum as

$$
p_{\mu}=m v_{\mu}+l_{\mu}
$$

with $v_{\mu}$ the four-velocity satisfying $v^{2}=1$ and $l_{\mu}$ a small off-shell momentum, $v \cdot l \ll m$ (for the present discussion we can set $l_{\mu}=0$ ). One can now construct eigenstates of the velocity projection operator $P_{v}=(1+\psi) / 2$ via

$$
\begin{aligned}
& \Psi=\mathrm{e}^{-i m v \cdot x}(H+h) \\
& \psi H=H, \quad \psi h=-h
\end{aligned}
$$


which in the nucleon rest-frame $v_{\mu}=(1,0,0,0)$ leads to the standard non-relativistic reduction of a spinor into upper and lower components. Substituting (6.22) into (6.20) one finds

$$
\mathcal{L}=\bar{H}(i v \cdot \partial) H-\bar{h}(i v \cdot \partial+2 m) h+\bar{H} i \partial^{\perp} h+\bar{h} i \partial^{\perp} H
$$

with $\partial^{\perp}$ the transverse part of the Dirac operator, $\partial=\psi(v \cdot \partial)+\partial^{\perp}$. Form eq.(6.23) it follows immediately that the large component field $H$ obeys a free Dirac equation

$$
v \cdot \partial H=0
$$

modulo corrections which are suppressed by powers of $1 / \mathrm{m}$. A more elegant path integral formulation is given by Mannel et al. [151]. There is one more point worth noticing. In principle, the field $H$ should carry a label ' $v$ ' since it has a definite velocity. The Lorentz invariant Lagrangian $\mathcal{L}$ follows from $\mathcal{L}_{v}=\mathcal{L}\left[\bar{H}_{v}, H_{v}\right]$ via a suitable integration,

$$
\mathcal{L}=\int \frac{d^{3} v}{2 v_{0}} \mathcal{L}_{v}
$$

This is of importance when the incoming and outgoing baryons do not have the same velocity. For most of the purposes to be discussed we do not need to worry about the label ' $v$ ' and will therefore drop it.

Let me now return to the $\pi N$ system. The reasoning is completely analogous to the one just discussed. I follow here the systematic analysis of quark currents in flavor $S U(2)$ of Bernard et al. [152]. Starting from eq.(6.11) we write $\Psi$ in the form of eq.(6.22) and eliminate the small component field $h$ via its equation of motion,

$$
h=\frac{1}{2}(1-\psi) \frac{1}{2 \stackrel{\circ}{m}}\left(i \not D+\frac{\stackrel{\circ}{g}_{A}}{2} \not \gamma_{5}\right) H+\mathcal{O}\left(1 / \stackrel{\circ}{m}^{2}\right)
$$

This leads to

$$
\begin{aligned}
\mathcal{L}_{\pi N}^{(1)}= & \bar{H}\left(i v \cdot D+\stackrel{\circ}{g}_{A} S \cdot u\right) H \\
& +\frac{1}{2 \stackrel{\circ}{m}} \bar{H}\left(i \not D+\frac{\stackrel{\circ}{g}_{A}}{2} \not \gamma \gamma_{5}\right) \frac{1-\psi}{2}\left(i \not D+\frac{\stackrel{\circ}{g}_{A}}{2} \not \gamma \gamma_{5}\right) H+\mathcal{O}\left(1 / \stackrel{\circ}{m}^{2}\right)
\end{aligned}
$$

As advertised, the nucleon mass term has disappeared. Furthermore, any Dirac bilinear $\bar{\Psi} \Gamma_{\mu} \Psi\left(\Gamma_{\mu}=1, \gamma_{\mu}, \gamma_{5}, \ldots\right)$ can be expressed in terms of the velocity $v_{\mu}$ and the spinoperator $2 S_{\mu}=i \gamma_{5} \sigma_{\mu \nu} v^{\nu}$. It obeys the relations

$$
S \cdot v=0, S^{2}=-\frac{3}{4},\left\{S_{\mu}, S_{\nu}\right\}=\frac{1}{2}\left(v_{\mu} v_{\nu}-g_{\mu \nu}\right), \quad\left[S_{\mu}, S_{\nu}\right]=i \epsilon^{\mu \nu \alpha \beta} v_{\alpha} S_{\beta}
$$

Using the convention $\epsilon^{0123}=-1$, we can rewrite the standard Dirac bilinears as $[147$, 152]:

$$
\begin{aligned}
& \bar{H} \gamma_{\mu} H=v_{\mu} \bar{H} H, \bar{H} \gamma_{5} H=0, \bar{H} \gamma_{\mu} \gamma_{5} H=2 \bar{H} S_{\mu} H \\
& \bar{H} \sigma^{\mu \nu} H=2 \epsilon^{\mu \nu \alpha \beta} v_{\alpha} \bar{H} S_{\beta} H, \bar{H} \gamma_{5} \sigma^{\mu \nu} H=2 i\left(v^{\mu} \bar{H} S^{\nu} H-v^{\nu} \bar{H} S^{\mu} H\right)
\end{aligned}
$$


Therefore, the Dirac algebra is extremely simple in the extreme non-relativistic limit. The disappearance of the nucleon mass term to leading order in $1 / \stackrel{\circ}{m}$ now allows for a consistent chiral power counting. The tree level Lagrangian is of order $\mathcal{O}(q)$ and oneloop diagrams contribute to order $\mathcal{O}\left(q^{3}\right)$, with $q$ now a genuine small momentum (for the baryons, only the small $l_{\mu}$ counts). The power counting scheme is discussed in detail by Jenkins and Manohar [149] based on the chiral quark model approach of Manohar and Georgi [20]. In essence, since all momenta can be considered small compared to the chiral scale $\Lambda \simeq M_{\rho}$ one has a scheme as in the meson sector, with the main difference that odd powers in $q$ are allowed here. An important point is that all vertices now consist of a string of operators with increasing powers in $1 / \mathrm{m}$. For the calculation of one-loop diagrams, one needs, however, only the mass independent propagator and vertices, we have for example

$$
\begin{array}{ll}
\text { Nucleon propagator : } & S(\omega)=\frac{i}{v \cdot l+i \eta}, \eta>0 \\
\text { Photon - nucleon vertex : } \quad i e \frac{1+\tau_{3}}{2} \epsilon \cdot v+\mathcal{O}(1 / \stackrel{\circ}{m}) \\
\text { Pion - nucleon vertex : } \quad\left(\stackrel{\circ}{g}_{A} / F\right) \tau^{a} S \cdot q+\mathcal{O}(1 / \stackrel{\circ}{m})
\end{array}
$$

with $\omega=v \cdot l$. For the contact terms, one therefore has to go further in the $1 / m$ expansion to achieve the same accuracy. This means that in case of a one-loop calculation one has to take into account all local contact terms which contribute to the order the loops do. A consequence is that the effective Lagrangian written down by Jenkins and Manohar [147] is not complete since it only contains terms with one derivative or one quark mass insertion, i.e. $\mathcal{L}_{\pi N}^{(1)}$ and parts of $\mathcal{L}_{\pi N}^{(2)}$. However one should also account for $\mathcal{L}_{\pi N}^{(3)}$. To make this statement more transparent, consider the isovector anomalous magnetic moment of the nucleon. The isovector-vector quark current can be expressed in terms of two form factors. In the heavy mass limit, this is most conveniently done in the Breit frame where the photon transfers no energy since it allows for a unique translation of Lorentz-invariant matrix elements into non-relativistic ones. For the case at hand, this gives

$$
\begin{aligned}
<N\left(p^{\prime}\right)\left|\bar{q} \gamma_{\mu} \tau_{3} q\right| N(p)>= & \left\{F_{1}^{V}(t)+\frac{t}{4 m_{N}^{2}} F_{2}^{V}(t)\right\} v_{\mu} \bar{H} \tau_{3} H \\
& +\frac{1}{m_{N}}\left\{F_{2}^{V}(t)+F_{1}^{V}(t)\right\} \bar{H}\left[S_{\mu}, S \cdot\left(p^{\prime}-p\right)\right] \tau_{3} H .
\end{aligned}
$$

Consider now the Pauli form factor $F_{2}^{V}(t)$. At zero momentum transfer, it gives the isovector anomalous magnetic moment, $F_{2}^{V}(0)=\kappa_{V}=\kappa_{p}-\kappa_{n}=3.71$. The calculation of $F_{2}^{V}(t)$ is straightforward [152], at $t=0$ one finds

$$
\kappa_{V}=c_{6}^{\prime}-\frac{\stackrel{\circ}{g}_{A}^{2} M \stackrel{\circ}{m}}{4 \pi F^{2}}
$$


where the second term on the r.h.s. of (6.32) is the one-loop contribution (which is nonanalytic in the quark mass since $M \sim \sqrt{\hat{m}}$ [155]) and the constant $c_{6}^{\prime}$ has to be identified with the anomalous isovector magnetic moment in the chiral limit. It originates from a counterterm of $\mathcal{L}_{\pi N}^{(2)}$,

$$
\mathcal{L}_{\pi N}^{(2)}=\frac{c_{6}^{\prime}+1}{4 \stackrel{\circ}{m}} \epsilon^{\mu \nu \alpha \beta} v_{\mu} \bar{H} S_{\nu} f_{\alpha \beta}^{+} H
$$

where the field strength tensor $f_{\alpha \beta}^{+}$is defined via $f_{\alpha \beta}^{+}=u^{\dagger} F_{\alpha \beta} u^{\dagger}+u F_{\alpha \beta} u$ with $F_{\alpha \beta}$ the conventional photon fields strength tensor. Choosing $c_{6}^{\prime}=5.62$, one reproduce the empirical value of $\kappa_{V}$. This value of $\stackrel{\circ}{\kappa}_{V}=c_{6}^{\prime}$ is close to what one would estimate if one generates the contact term (6.33) from $\rho$-exchange since the tensor coupling of the $\rho$ is about $\kappa_{\rho} \simeq 6$. Other observables and the interplay between the loop and counterterm contributions are discussed in ref.[152].

Another topic is the matching to the relativistic formalism. This has been addressed in ref.[152]. The underlying assumption is that the extreme non-relativistic formulation should match to the relativistic one (this point of view is not shared by everybody). In that case, one can derive matching conditions between the various low-energy constants. This allows one to use previously determined values of these coefficients also in the non-relativistic approach. In fact, these low-energy constants depend on the scale of dimensional regularization, called $\lambda$ here. To be specific, consider the wave-function renormalization ( $Z$-factor) of the nucleon. It is defined by the residue of the propagator at the physical mass pole,

$$
\frac{i}{p \cdot v-\stackrel{\circ}{m}-\Sigma(\omega)} \rightarrow \frac{i Z_{N}}{p \cdot v-m_{N}}
$$

with $\Sigma(\omega)$ the nucleon self-energy in the one-loop approximation. One finds

$$
Z_{N}=1+\Sigma^{\prime}(0)=1-\frac{3 \stackrel{\circ}{A}_{A}^{2} M^{2}}{32 \pi^{2} F^{2}}\left[3 \ln \frac{M}{\lambda}+1\right]-\frac{4 M^{2}}{F^{2}} b^{\prime r}{ }_{12}^{r}(\lambda)
$$

where the coupling constant $b_{12}^{\prime}$ has eaten up the infinity from the loop $[142,152]$. If one compares (6.35) to the expression of GSS [142] expanded in powers of $\mu=M / \stackrel{\circ}{m}$, one finds

$$
Z_{N}^{r e l}=1-\frac{3 \stackrel{\circ}{g}_{A}^{2} M^{2}}{32 \pi^{2} F^{2}}\left[\ln \frac{M}{\lambda}+2 \ln \mu+2\right]-\frac{4 M^{2}}{F^{2}} b_{12}^{r}(\lambda)+\mathcal{O}\left(\mu^{3}\right)
$$

Therefore, the heavy mass and the relativistic theory only match if one sets

$$
\begin{aligned}
\lambda & =\stackrel{\circ}{m} \\
b_{12}^{\prime} & =b^{\prime r}{ }_{12}(\lambda)+\gamma^{\prime b}{ }_{12} L, \quad \gamma_{12}^{\prime b}=-\frac{9}{8} \stackrel{\circ}{g}_{A}^{2}
\end{aligned}
$$


The result $\lambda=\stackrel{\circ}{m}$ is not quite unexpected since one has integrated out the field $h$ of mass $2 m$. Therefore, if one chooses to retain only the leading non-analytic contributions from the loops (as it is often done), one has to set $\lambda=\stackrel{\circ}{m}$. However, as stressed many times before, an accurate CHPT calculation at a given order has to account for all loop and counterterm contributions to the order one is working. A more detailed discussion of the interplay between the relativistic and the heavy mass formulation is given in ref.[152].

One particular advantage of the heavy mass formulation is the fact that it is very easy to include the baryon decuplet, i.e. the spin- $3 / 2$ states. This has been done in full detail by Jenkins and Manohar [148]. The inclusion of the $\Delta(1232)$ is motivated mostly by the following argument: The $N \Delta$ mass-splitting $m_{\Delta}-m_{N}$ is only about twice as much as the pion mass, so that one expects significant contributions from this close-by resonance (the same holds true for the full decuplet in relation to the octet). This expectation is borne out in many phenomenological models. However, it should be stressed that if one chooses to include this baryon resonance (or the full decuplet), one again has to account for all terms of the given accuracy one aims at, say $\mathcal{O}\left(q^{3}\right)$ in a one-loop calculation. This tends to be overlooked in the presently available literature. Furthermore, the mass difference $m_{\Delta}-m_{N}$ does not vanish in the chiral limit thus destroying the consistent power counting (as it is the case with the baryon mass in the relativistic formalism discussed in section 6.1). I will not consider the full baryon octet coupled to the decuplet but will only focus on the physics of the $\pi N \Delta$ system. The decuplet fields are described by Rarita-Schwinger spinor $\left(\tilde{T}^{\mu}\right)_{a b c}$ with $a, b, c \in\{1,2,3\}$. This spinor contains both spin $-1 / 2$ and spin $-3 / 2$ components. The spin $-1 / 2$ pieces are projected out by use of the constraint $\gamma_{\mu} \tilde{T}^{\mu}=0$. Under $S U(3) \times S U(3) \tilde{T}^{\mu}$ transforms as $\left(\tilde{T}^{\mu}\right)_{a b c} \rightarrow(K)_{a}^{d}(K)_{b}^{e}(K)_{c}^{f}\left(\tilde{T}^{\mu}\right)_{d e f}$ and one defines a velocity dependent field via

$$
\tilde{T}^{\mu}=\mathrm{e}^{-i m_{\Delta} v \cdot x}(T+t)^{\mu}
$$

with $m_{\Delta}$ the $\Delta(1232)$ mass (in general one should use the average decuplet mass). In terms of the physical states we have $T_{111}=\Delta^{++}, T_{112}=\Delta^{+} / \sqrt{3}, T_{122}=\Delta^{0} / \sqrt{3}$, $T_{222}=\Delta^{-}$. The effective $\Delta N \pi$ Lagrangian to leading order reads

$$
\mathcal{L}_{\Delta N \pi}^{(1)}=-i \bar{T}^{\mu} v \cdot D T_{\mu}+\Delta \bar{T}^{\mu} T_{\mu}+\frac{3 \stackrel{\circ}{g}_{A}}{2 \sqrt{2}}\left(\bar{T}^{\mu} u_{\mu} H+\bar{H} u_{\mu} T^{\mu}\right)
$$

with $\Delta=m_{\Delta}-m_{N}$. Clearly one is left with some residual mass dependence from the $N \Delta$-splitting (notice that the average octet-decuplet splitting is smaller than $\Delta$ ). In the language of ref.[148] we have set $\mathcal{C}=3 \stackrel{\circ}{g}_{A} / 2$ which is nothing but the coupling constant relation $g_{\Delta N \pi}=3 g_{\pi N} / \sqrt{2}=1.89$. Empirically this relation is fulfilled within a few per cent. From the width of the decay $\Delta \rightarrow N \pi$ one has $\mathcal{C}=1.8$ [149], consistent with the value given before (if one uses the full decuplet the value of $\mathcal{C}$ reduces to 1.5). The propagator of the spin- $3 / 2$ fields reads

$$
S_{\Delta}(\omega)=i \frac{v^{\mu} v^{\nu}-g^{\mu \nu}-\frac{4}{3} S^{\mu} S^{\nu}}{\omega-\Delta}
$$


For all practical purposes, it is most convenient to work in the rest-frame $v_{\mu}=(1,0,0,0)$. In that case, one deals with the well-known non-relativistic isobar model which is discussed in detail in the monograph by Ericson and Weise [154]. We will come back to some calculations involving the $\Delta(1232)$ (or the full decuplet) in the following section.

A last comment on the heavy fermion EFT is necessary. While it is an appealing framework, one should not forget that the nucleon (baryon) mass is not extremely large. Therefore, one expects significant corrections from $1 / m$ suppressed contributions to many observables. This will become more clear in the discussion of threshold pion photo- and electroproduction. It is conceivable that going to one-loop order $\mathcal{O}\left(q^{3}\right)$ is not sufficient to achieve a very accurate calculation. This means that even more than in the meson sector higher-loop calculations should be performed to learn about the convergence of the chiral expansion. Ultimately, one might want to include more information in the unperturbed Hamiltonian. At present, it is not known how to do that but it should be kept in mind.

\subsection{Photo-nucleon processes}

In this section, I will be concerned with reactions involving photons, nucleons and pions. There has been much impetus from the experimental side in the recent years through precise measurements of the nucleons electromagnetic polarizabilities or the recent data on threshold pion photo- and electroproduction.

Let us first consider nucleon Compton scattering at low energies. The energy expansion of the spin-averaged Compton amplitude in the rest frame takes the form

$$
T(\gamma N \rightarrow \gamma N)=-\frac{e^{2} Z^{2}}{4 \pi m}+\bar{\alpha} \omega^{\prime} \omega \vec{\epsilon}^{\prime} \cdot \vec{\epsilon}+\bar{\beta}\left(\vec{\epsilon}^{\prime} \times \vec{k}^{\prime}\right) \cdot(\vec{\epsilon} \times \vec{k})+\mathcal{O}\left(\omega^{4}\right)
$$

with $(\omega, \vec{k}, \vec{\epsilon})$ and $\left(\omega^{\prime}, \vec{k}^{\prime}, \vec{\epsilon}^{\prime}\right)$ the frequencies, momenta and polarization vectors of the incoming and outgoing photon, respectively. The first term, which is energy-independent, is nothing but the Thomson scattering amplitude as mandated by gauge invariance. It constitutes a low-energy theorem as the photon energy tends to zero. At next-toleading order in the energy expansion, the photon probes the non-trivial structure of the spin-1/2 particle (here, the nucleon) it scatters off. This information is encoded in two structure constants, the so-called electric $(\bar{\alpha})$ and magnetic $(\bar{\beta})$ polarizabilities. These have been determined rather accurately over the last years [155]

$$
\begin{aligned}
& \bar{\alpha}_{p}=10.8 \pm 1.0 \pm 1.0, \quad \bar{\beta}_{p}=3.4 \mp 1.0 \mp 1.0 \\
& \bar{\alpha}_{n}=12.3 \pm 1.5 \pm 2.0, \quad \bar{\beta}_{n}=3.1 \mp 1.6 \mp 2.0
\end{aligned}
$$

all in units of $10^{-4} \mathrm{fm}^{-3}$ which I will use throughout. The errors in (6.42) are anticorrelated since one imposes the well-established dispersion sum rule results $(\bar{\alpha}+\bar{\beta})_{p}=$ $14.2 \pm 0.3$ and $(\bar{\alpha}+\bar{\beta})_{n}=15.8 \pm 0.5[156]$. So the proton and the neutron behave essentially as electric dipoles and their respective sum of the polarizabilities is approximately 
equal. For calculating the em polarizabilities, it is most convenient to consider the spin-averaged Compton tensor in forward direction $[157,158]$

$$
\begin{aligned}
\theta_{\mu \nu} & =\frac{1}{4} \operatorname{Tr}\left\{(\not p+m) \int d^{4} x e^{i k \cdot x}<N(p)\left|T J_{\mu}^{e m}(x) J_{\nu}^{e m}(0)\right| N(p)>\right\} \\
& =e^{2}\left\{g_{\mu \nu} A(s)+k_{\mu} k_{\nu} B(s)+\left(p_{\mu} k_{\nu}+p_{\nu} k_{\mu}\right) C(s)+p_{\mu} p_{\nu} D(s)\right\}
\end{aligned}
$$

The pertinent Mandelstam variables are $s=(p+k)^{2}$ and $u=2 m^{2}-s$ since $t=0$ in forward direction. Gauge invariance $\left(k_{\mu} \Theta^{\mu \nu}=0\right)$ and $u \leftrightarrow s$ crossing symmetry reduce the number of independent scalar functions in (6.43) to two. It is straightforward to read off the polarizabilities

$$
\bar{\alpha}+\bar{\beta}=-\frac{e^{2} m}{2 \pi} A^{\prime \prime}\left(m^{2}\right), \bar{\beta}=-\frac{e^{2}}{4 \pi m} B\left(m^{2}\right)
$$

where the prime denotes differentiation with respect to $s$. The non-renormalization theorem of the electric charge leads to the additional constraint $A\left(m^{2}\right)=Z^{2}$.

To one-loop in CHPT, the em polarizabilities are pure loop effects. This means that there are no contributions from the contact terms $\mathcal{L}_{\pi N}^{(2,3)}$. Therefore, no undetermined low-energy constants enter the final results and one tests the loop structure of chiral QCD. In the relativistic formalism, the calculation of $\bar{\alpha}_{p, n}$ and $\bar{\beta}_{p, n}$ has been performed by Bernard et al. in refs.[157,158]. Although one has to evaluate 52/22 Feynman diagrams for the proton/neutron, the final results are simple one dimensional integrals due to the particular kinematics. In chiral $S U(2)$, the expansion of the polarizabilities in powers of $\mu=M_{\pi} / m$ (ratio of the pion and the nucleon mass) takes the form

$$
\begin{aligned}
& \bar{\alpha}_{p}=C\left\{\frac{5 \pi}{2 \mu}+18 \ln \mu+\frac{33}{2}+\mathcal{O}(\mu)\right\} \\
& \bar{\alpha}_{n}=C\left\{\frac{5 \pi}{2 \mu}+6 \ln \mu-\frac{3}{2}+\mathcal{O}(\mu)\right\} \\
& \bar{\beta}_{p}=C\left\{\frac{\pi}{4 \mu}+18 \ln \mu+\frac{63}{2}+\mathcal{O}(\mu)\right\} \\
& \bar{\beta}_{n}=C\left\{\frac{\pi}{4 \mu}+6 \ln \mu+\frac{5}{2}+\mathcal{O}(\mu)\right\}
\end{aligned}
$$

with $C=e^{2} g_{\pi N}^{2} / 192 \pi^{3} m^{3} \simeq 0.26 \cdot 10^{-4} \mathrm{fm}^{3}$. To leading order, the polarizabilities diverge as $1 / M_{\pi}$ as $M_{\pi}$ tends to zero. This is an expected result since the pion cloud becomes long-ranged in the chiral limit, i.e. the Yukawa suppression factors turn into a simple power law fall-off. In fact, in the heavy mass formalism, it is only this most singular term which results from the one-loop calculation [152]. Working in the Coulomb gauge $\epsilon \cdot v=0$, it is easy to filter out the diagrams which lead to this singularity. 
These diagrams were also found in the relativistic formalism, however, after tedious calculations. The most singular term reproduces the data fairly well

$$
\begin{aligned}
& \bar{\alpha}_{p}=\bar{\alpha}_{n}=\frac{5 e^{2} g_{\pi N}^{2}}{384 \pi^{2} m^{2}} \frac{1}{M_{\pi}}=13.6 \\
& \bar{\beta}_{p}=\bar{\beta}_{n}=\bar{\alpha}_{p} / 10=1.4
\end{aligned}
$$

i.e. $(\bar{\alpha}+\bar{\beta})_{p}=(\bar{\alpha}+\bar{\beta})_{n}=14.9$ close to the empirical values and, furthermore, $\bar{\alpha}_{p, n} \gg$ $\bar{\beta}_{p, n}$. Some of the $1 / m$ corrections modifying these leading order results are resummed in the relativistic calculation, they tend to decrease the polarizabilities and lead to too small values for the sums $[157,158]$. However, there is also the contribution from the $\Delta(1232)$ which is important for $\bar{\beta}_{p, n}$ in many phenomenological models. Clearly, the $1 / m$ suppressed terms lead to isospin breaking $\left(\bar{\alpha}_{p} \neq \bar{\alpha}_{n}, \bar{\beta}_{p} \neq \bar{\beta}_{n}\right)$. In a recent paper [159] the $\ln \mu$-term in (6.45) was criticised as incorrect since the nucleon mass appears in the logarithm. This is, however, a false statement - from the discussion of the matching condition $\lambda=m$ it should be clear that such terms are indeed possible. Clearly, they are not the whole story at order $q^{4}$. When one extends the calculation to flavor $S U(3)$, one has to consider pion and kaon loops. This was for the first time done in ref.[160], where also predictions for the electromagnetic polarizabilities of the hyperons are given. For typical values of the $S U(3) F$ and $D$ couplings (see appendix B), the kaon loops tend to increase $\bar{\alpha}_{p}$ over $\bar{\alpha}_{n}$ by about 15 per cent. A similar observation was also made in ref.[159]. In that paper, in addition the contribution from the decuplet $\left(\Delta, \Sigma, \Sigma^{\star}\right)$ in the intermediate states were considered (the $\Delta$-loop contribution had previously been given by Kaiser [161]). The authors of ref.[159] finds $\bar{\alpha}_{p}=14.1, \bar{\alpha}_{n}=13.4, \bar{\beta}_{p}=0.2$ and $\bar{\beta}_{n}=0.1$ not too different from the leading order results (6.46) (clearly, the magnetic polarizabilities are most affected by the decuplet contribution). However, unrealistically small values for the $F$ and $D$ coupling constants are used in this calculation. What has to be done here is to perform a full-fledged calculation to include all terms up-toand-including $\mathcal{O}\left(q^{4}\right)$. This is possible in the heavy mass formalism and is the only way to get a clean handle at the terms modifying the leading order $\left(q^{3}\right)$ result $(6.46)$. In ref.[152] a prediction was also made for the spin-dependent Compton amplitude $f_{2}(\omega)$ at low $\omega$ and in ref.[162], the small momentum expansion of the Drell-Hearn-Gerasimov sum rule was investigated. These predictions become relevant when the scattering of polarized photons on polarized nucleons will be performed. For further details, the reader is referred to refs.[152,162].

Next, let me consider threshold pion photo- and electroproduction. In the threshold region, i.e. when the real photon has just enough energy to produce the pion at rest, the differential cross section for the process $\gamma(k)+p\left(p_{1}\right) \rightarrow \pi^{0}(q)+p\left(p_{2}\right)$ can be expressed in terms of the electric dipole amplitude $E_{0+}, d \sigma / d \Omega=\left(E_{0+}\right)^{2}$ as $\vec{q}$ tends to zero. This multipole is of particular interest since in the early seventies a low-energy theorem (LET) for neutral pion production was derived [163]. The recent measurements at Saclay and Mainz [164] seemed to indicate a gross violation of this LET, which predicts $E_{0+}=$ 
$-2.3 \cdot 10^{-3} / M_{\pi^{+}}$at threshold. However, the LET was reconsidered (and rederived) and the data were reanalyzed, leading to $E_{0+}=(-2.0 \pm 0.2) \cdot 10^{-3} / M_{\pi^{+}}$in agreement with the LET prediction. These developments have been subject of a recent review by Drechsel and Tiator [165]. Therefore, I will focus here on the additional insight gained from CHPT calculations. In fact, the LET for neutral pion photoproduction at threshold is an expansion in powers of $\mu=M_{\pi} / m \sim 1 / 7$ and predicts the coefficients of the first two terms in this series, which are of order $\mu$ and $\mu^{2}$, respectively, in terms of measurable quantities like the pion-nucleon coupling constant $g_{\pi N}$, the nucleon mass $m$ and the anomalous magnetic moment of the proton, $\kappa_{p}$. In ref.[166] it was, however, shown that a certain class of loop diagrams modifies the LET at next-to-leading order $\mathcal{O}\left(\mu^{2}\right)$. This analysis was extended to threshold pion electroproduction $\gamma^{\star} p \rightarrow \pi^{0} p$ in ref.[167]. In the electroproduction case, there is another $S$-wave multipole called $L_{0+}$ due to the longitudinal coupling of the virtual photon to the nucleon. The resulting expressions for $E_{0+}$ and $L_{0+}$ to order $\mathcal{O}\left(\mu^{2}\right)$ and $\mathcal{O}(\nu)$, with $\nu=-k^{2} / m^{2}>0\left(k^{2}<0\right.$ in the electroproduction reaction) read (notice that $\mu$ is of order $q$ whereas $\nu$ is of order $\left.q^{2}\right)$ :

$$
\begin{aligned}
& E_{0+}=C\{-\mu+\mu^{2}\left[\frac{1}{2}\left(3+\kappa_{p}\right)+\frac{m^{2}}{4 \pi F_{\pi}^{2}} \xi_{2}\left(-\nu / \mu^{2}\right)\right] \\
&\left.-\nu\left[\frac{1}{2}\left(1+\kappa_{p}\right)+\frac{m^{2}}{8 \pi F_{\pi}^{2}}\left[2 \xi_{2}\left(-\nu / \mu^{2}\right)-\xi_{1}\left(-\nu / \mu^{2}\right)\right]\right]+\mathcal{O}\left(q^{3}\right)\right\} \\
& L_{0+}=C\left\{-\mu+\mu^{2}\left[\frac{3}{2}+\frac{m^{2}}{8 \pi F_{\pi}^{2}} \xi_{1}\left(-\nu / \mu^{2}\right)\right]-\frac{\nu}{2}++\mathcal{O}\left(q^{3}\right)\right\}
\end{aligned}
$$

with $C=e g_{\pi N} / 8 \pi m=0.17 \mathrm{GeV}^{-1}$. The transcendental functions $\xi_{1,2}(\alpha), \alpha=\nu / \mu^{2}$ can not be further expanded in $\mu$ and $\nu$ separately. They are given by:

$$
\begin{aligned}
& \xi_{1}(\alpha)=\frac{1}{1+\alpha}+\frac{\alpha}{2(1+\alpha)^{3 / 2}}\left[\frac{\pi}{2}+\arcsin \frac{\alpha}{2+\alpha}\right] \\
& \xi_{2}(\alpha)=\frac{3 \alpha}{4(1+\alpha)^{2}}+\frac{4+4 \alpha+3 \alpha^{2}}{8(1+\alpha)^{5 / 2}}\left[\frac{\pi}{2}+\arcsin \frac{\alpha}{2+\alpha}\right]
\end{aligned}
$$

At the photon point $k^{2}=0$, one has $\nu=0$ and $\xi_{1}(0)=1, \xi_{2}(0)=\pi / 4$. Therefore, to order $\mu^{2}$ one has a term $\sim\left(m / 4 F_{\pi}\right)^{2}$ which modifies the LET of ref.[163]. To order $\mu^{2}$ and $\nu$, the form (6.48) is the correct one as given by QCD. The origin of these novel contributions proportional to $\xi_{1,2}(\alpha)$ can most easily be traced back in the heavy mass formalism. In ref.[152] it was shown that working in the Coulomb gauge and the proton rest frame, only four diagrams are non-vanishing for $\pi^{0}$ threshold production. These are the so-called triangle and rescattering diagrams and their crossed partners. The contribution of the triangle diagram is non-analytic in the quark mass, $\Delta A_{1} \sim M_{\pi} \sim$ $\sqrt{\hat{m}}[166]$. This non-analyticity is generated by the loop integral associated to this 
Fig. 13: a) Total cross section for $\gamma p \rightarrow \pi^{0} p$ from the one-loop calculation of Ref.[169]. The tree and one-loop results are indicated by the dashed and solid line, respectively. The data are from the Saclay (closed circles) and Mainz (open circles) groups [164]. b) Same with the inclusion of isospin-breaking in comparison with the Mainz data. Notice the weak cusp at $\pi^{+} n$ threshold $\left(E_{\gamma}=151.4 \mathrm{MeV}\right)$. After the $\pi^{+} n$ threshold, the predicted total cross section agrees with the one given in Fig.13a.

diagram, it develops a logarithmic singularity in the chiral limit. Therefore, the naive arguments based on $s \leftrightarrow u$ crossing which lead to $\Delta A_{1} \sim M_{\pi}^{2}$ are invalid. To see this effect, one has to calculate loop diagrams (this was known to Vainsthein and Zakharov [163], but they unfortunately calculated the harmless rescattering diagram). Notice also that the anomalous magnetic moment $\kappa_{p}$ is generated by loops and counterterms in CHPT. It would therefore be surprising if that would be the only such contribution and it is not. As a final comment on eq.(6.47) let me point out that the LETs contain no photon-nucleon form factors [168]. These are build up order by order in the chiral expansion and to order $\mathcal{O}\left(q^{2}\right)$, one only sees their leading terms. In ref.[169], a complete analysis of threshold pion photoproduction was given based on the relativistic formalism. 
This is necessary since in the static limit one only gets the terms including order $\mu^{2}$ in $E_{0+}$. A quick look at (6.47) reveals that the coefficient of the $\mu^{2}$ term is large and so the leading order term $\sim \mu$ is cancelled. This means that the expansion in $\mu$ is slowly converging and one has to go beyond the extreme non-relativistic limit. The total cross section for $\gamma p \rightarrow \pi^{0} p$ in the threshold region is shown in fig.13a. It agrees reasonably well with the data. In that paper, one can also find a prediction for the cross section of $\gamma n \rightarrow \pi^{0} n$. This process is even more sensitive to the chiral symmetry breaking since for the electric dipole amplitude at threshold, $E_{0+} \sim \mathcal{O}\left(\mu^{2}\right)$. A measurement of this reaction is intended using a deuteron target [170]. In the one-loop approximation used in these calculations, one has no isospin-breaking and therefore $M_{\pi^{0}}=M_{\pi^{+}}$and $m_{p}=m_{n}$. To study some of the effects of isospin-breaking, in ref.[171] a calculation was presented in which the physical masses for the charged and neutral pions as well as for the proton and neutron were used (this is not a complete calculation since at this order there are many other effects). In that case, one has two thresholds, $\pi^{0} p$ and $\pi^{+} n$, separated by $\simeq 5 \mathrm{MeV}$. The cross section in this energy regime is shown in fig.13b. It is clearly improved compared to the one-loop prediction. This indicates that a calculation beyond the isosymmetric one-loop approximation should be performed. Very recently, new accurate data very close to threshold have become available for the electroproduction process $\gamma^{\star} p \rightarrow \pi^{0} p$ [172]. In particular, the $S$-wave cross section $a_{0}$, which is a particular combination of $E_{0+}$ and $L_{0+}$, was analyzed (for the first time in this kinematical regime). A one loop CHPT calculation was performed by Bernard et al. [173] and it was shown that loop effects are indeed necessary to explain the data as shown in fig. 14 .

Fig. 14: The S-wave component of the neutral pion electroproduction cross section, calculated from CHPT (solid line) and PV (dotted line) are compared. The kinematics is $W=1074 \mathrm{MeV}$ and $\epsilon=0.58$ [173]. The data extracted in ref.[172] are also shown. 
Consider now the photoproduction of charged pions. In that case, there exists the LET due to Kroll and Ruderman [174] for the electric dipole amplitude (for $\gamma p \rightarrow \pi^{+} n$ and $\left.\gamma n \rightarrow \pi^{-} p\right)$. The predictions are in agreement with the data. The loop corrections are much more tame than in the case of neutral pion production, they start at order $\mathcal{O}\left(\mu^{2}, \mu^{2} \ln \mu^{2}\right)$ whereas the leading term is of order one. In the case of charged pion electroproduction, an interesting observation was made in ref.[175]. The starting point is the venerable LET due to Nambu, Lurié and Shrauner [176] for the isospin-odd electric dipole amplitude $E_{0+}^{(-)}$in the chiral limit,

$$
E_{0+}^{(-)}\left(M_{\pi}=0, k^{2}\right)=\frac{e g_{A}}{8 \pi F_{\pi}}\left\{1+\frac{k^{2}}{6} r_{A}^{2}+\frac{k^{2}}{4 m^{2}}\left(\kappa_{V}+\frac{1}{2}\right)+\mathcal{O}\left(k^{3}\right)\right\}
$$

Therefore, measuring the reactions $\gamma^{\star} p \rightarrow \pi^{+} n$ and $\gamma^{\star} n \rightarrow \pi^{-} p$ allows to extract $E_{0+}^{(-)}$ and one can determine the axial radius of the nucleon, $r_{A}$. This quantity measures the distribution of spin and isospin in the nucleon, i.e. probes the Gamov-Teller operator $\vec{\sigma} \cdot \vec{\tau}$. A priori, the axial radius is expected to be different from the typical electromagnetic size, $r_{\mathrm{em}} \simeq 0.8 \mathrm{fm}$. It is customary to parametrize the axial form factor $G_{A}\left(k^{2}\right)$ by a dipole form, $G_{A}\left(k^{2}\right)=\left(1-k^{2} / M_{A}^{2}\right)^{-2}$ which leads to the relation $r_{A}=\sqrt{12} / M_{A}$. The axial radius determined from electroproduction data is typically $r_{A}=0.59 \pm 0.04 \mathrm{fm}$ [177] whereas (anti)neutrino-nucleon reactions lead to somewhat larger values, $r_{A}=$ $0.65 \pm 0.03 \mathrm{fm}$. This discrepancy is usually not taken seriously since the values overlap within the error bars. However, it was shown in ref.[175] that pion loops modify the LET (6.49) at order $k^{2}$ for finite pion mass. In the heavy mass formalism, the coefficient of the $k^{2}$ term reads

$$
\frac{1}{6} r_{A}^{2}+\frac{1}{4 m^{2}}\left(\kappa_{V}+\frac{1}{2}\right)+\frac{1}{128 F_{\pi}^{2}}\left(1-\frac{12}{\pi^{2}}\right)
$$

where the last term in (6.50) is the new one. This means that previously one had extracted a modified radius, the correction being $3\left(1-12 / \pi^{2}\right) / 64 F_{\pi}^{2} \simeq-0.046 \mathrm{fm}^{2}$. This closes the gap between the values of $r_{A}$ extracted from electroproduction and neutrino data. It remains to be seen how the $1 / \mathrm{m}$ suppressed terms will modify the result (6.50). Such investigations are underway.

\subsection{Baryon masses and the $\sigma$-term}

In this section, I will mostly be concerned with some recent work on the so-called pion-nucleon $\sigma$-term related to low-energy $\pi N$ scattering. As an entrée, however, it is mandatory to briefly discuss the chiral expansion of the baryon masses. As we will see later, the $\sigma$-term is nothing but a nucleon mass shift related to the finiteness of the quark masses. 
Gasser [55] and Gasser and Leutwyler [3] were the first to systematically investigate the baryon masses at next-to-leading order. The quark mass expansion of the baryon masses takes the form

$$
m_{B}=m_{0}+\alpha \mathcal{M}+\beta \mathcal{M}^{3 / 2}+\gamma \mathcal{M}^{2}+\ldots
$$

The constant $m_{0}$ reminds us that the baryon masses do not vanish in the chiral limit. The non-analytic piece proportional to $\mathcal{M}^{3 / 2}$ was first observed by Langacker and Pagels [141]. If one retains only the terms linear in the quark masses, one obtains the GellMann-Okubo relation $m_{\Sigma}+3 m_{\Lambda}=2\left(m_{N}+m_{\Xi}\right)$ (which is fulfilled within 0.6 per cent in nature) for the octet and the equal spacing rule for the decuplet, $m_{\Omega}-m_{\Xi^{*}}=$ $m_{\Xi^{*}}-m_{\Sigma^{*}}=m_{\Sigma^{*}}-m_{\Delta}$ (experimentally, one has 142:145:153 MeV). However, to extract quark mass ratios from the expansion (6.51), one has to work harder. This was done in refs. $[3,55]$. The non-analytic terms were modelled by considering the baryons as static sources surrounded by a cloud of mesons and photons - truely the first calculation in the spirit of the heavy mass formalism. The most important result of this analysis was already mentioned in section 3.2, namely that the ration $R=\left(\hat{m}-m_{s}\right) /\left(m_{u}-m_{d}\right)$ comes out consistent with the value obtained from the meson spectrum. Jenkins [179] has recently repeated this calculation using the heavy fermion EFT of refs. $[147,148,149]$, including also the spin-3/2 decuplet fields in the EFT. She concludes that the success of the octet and decuplet mass relations is consistent with baryon CHPT as long as one includes the decuplet. Its contributions tend to cancel the large corrections from the kaon loops like $M_{K}^{2} \ln M_{K}^{2}$. The calculation was done in the isospin limit $m_{u}=m_{d}=0$ so that nothing could be said about the quark mass ratio $R$. One would like to see such a refined analysis.

Let me now turn to the $\pi N \sigma$-term. It is defined via

$$
\sigma=\frac{\hat{m}}{2 m}<p|\bar{u} u+\bar{d} d| p>=\hat{m} \frac{\partial m}{\partial \hat{m}}
$$

making use of the Feynman-Hellmann theorem and the proton state $\mid p>$ is normalized via $\left\langle p^{\prime} \mid p\right\rangle=2(2 \pi)^{3} p_{0} \delta\left(\vec{p}-\vec{p}^{\prime}\right)$. The quantity $\sigma$ can be calculated from the baryon spectrum. To leading order in the quark masses, one finds

$$
\begin{aligned}
\sigma & =\frac{\hat{m}}{m_{s}-\hat{m}} \frac{m_{\Xi}+m_{\Sigma}-2 m_{N}}{1-y}+\mathcal{O}\left(\mathcal{M}^{3 / 2}\right) \\
y & =\frac{2<p|\bar{s} s| p>}{<p|\bar{u} u+\bar{d} d| p>}
\end{aligned}
$$

where $y$ is a measure of the strange quark content of the proton. Setting $y=0$ as suggested by the OZI rule, one finds $\sigma=26 \mathrm{MeV}$. However, from the baryon mass 
analysis it is obvious that one has to include the $\mathcal{O}\left(\mathcal{M}^{3 / 2}\right)$ contributions and estimate the $\mathcal{O}\left(\mathcal{M}^{2}\right)$ ones. This was done by Gasser [55] leading to

$$
\sigma=\frac{35 \pm 5 \mathrm{MeV}}{1-y}=\frac{\sigma_{0}}{1-y}
$$

However, in $\pi N$ scattering one does not measure $\sigma$, but a quantity called $\Sigma$ defined via

$$
\Sigma=F_{\pi}^{2} \bar{D}^{+}\left(\nu=0, t=2 M_{\pi}^{2}\right)
$$

with the bar on $D$ denoting that the pseudovector Born terms have been subtracted, $\bar{D}=D-D_{\mathrm{pv}}$. The amplitudes $D^{ \pm}$are related to the more conventional $\pi N$ scattering amplitudes $A^{ \pm}$and $B^{ \pm}$via $D^{ \pm}=A^{ \pm}+\nu B^{ \pm}$, with $\nu=(s-u) / 4 m$. The superscript ' \pm ' denotes the isospin (even or odd). $D$ is useful since it is related to the total cross section via the optical theorem. The kinematical choice $\nu=0, t=2 M_{\pi}^{2}$ (which lies in the unphysical region) is called the Cheng-Dashen point [180]. The relation between $\Sigma$ and the $\pi N$ scattering data at low energies is rather complex, see e.g. Höhler [181] for a discussion or Gasser [182] for an instructive pictorial (given also in ref.[144]). Based on dispersion theory, Koch [183] found $\Sigma=64 \pm 8 \mathrm{MeV}$ (notice that the error only reflects the uncertainty of the method, not the one of the underlying data). Gasser et al. [184] have recently repeated this analysis and found $\Sigma=60 \mathrm{MeV}$ (for a discussion of the errors, see that paper). There is still some debate about this value, but in what follows I will use the central result of ref.[184]. Finally, we have to relate $\sigma$ and $\Sigma$. The relation of these two quantities is based on the LET of Brown et al. [185] and takes the following form at the Cheng-Dashen point:

$$
\begin{gathered}
\Sigma=\sigma+\Delta \sigma+\Delta R \\
\Delta \sigma=\sigma\left(2 M_{\pi}^{2}\right)-\sigma(0)
\end{gathered}
$$

$\Delta \sigma$ is the shift due to the scalar form factor of the nucleon, $<p^{\prime}|\hat{m}(\bar{u} u+\bar{d} d)| p>=$ $\bar{u}\left(p^{\prime}\right) u(p) \sigma(t)$, with $t=\left(p^{\prime}-p\right)^{2}$ and $\Delta R$ is related to a remainder not fixed by chiral symmetry. The latter was found to be very small by GSS [142], $\Delta R=0.4 \mathrm{MeV}$. The one-loop calculation of ref.[142] for $\Delta \sigma$ gave $4.6 \mathrm{MeV}$, and in the heavy mass formulation one finds $7.4 \mathrm{MeV}[152]$. Adding up the pieces, this would amount to $y \approx 0.3 \ldots 0.4$, i.e. a large strange quark content in the nucleon. However, since one is dealing with strong $S$-wave $\pi \pi$ and $\pi N$ interactions, the suspicion arises that the one-loop approximation is not sufficient, as already discussed in section 4.4. Therefore, Gasser et al. [184,186] have performed a dispersion-theoretical analysis tied together with CHPT constraints for the scalar form factor $\sigma(t)$. The resulting numerical value is

$$
\Delta \sigma=(15 \pm 0.5) \mathrm{MeV}
$$

which is a stunningly large correction to the one-loop result. If one parametrizes the scalar form factor as $\sigma(t)=1+r_{S}^{2} t+\mathcal{O}\left(t^{2}\right)$, this leads to $r_{S}^{2}=1.6 \mathrm{fm}^{2}$, substantially 
larger than the typical electromagnetic size. This means that the scalar operator $\hat{m}(\bar{u} u+$ $\bar{d} d$ ) sees a more extended pion cloud. Notice that for the pion, a similar enhancement of the scalar radius was already observed, $\left(r_{S}^{2} / r_{\mathrm{em}}^{2}\right)_{\pi} \simeq 1.4[13]$. Putting pieces together, one ends up with $\sigma=45 \pm 8 \mathrm{MeV}$ [184] to be compared with $\sigma_{0} /(1-y)=(35 \pm$ 5) $\mathrm{MeV} /(1-y)$. This means that the strange quarks contribute approximately $10 \mathrm{MeV}$ to the $\sigma$-term and thus the mass shift induced by the strange quarks is $m_{s}<p|\bar{s} s| p>\simeq$ $\left(m_{s} / 2 \hat{m}\right) \cdot 10 \mathrm{MeV} \simeq 130 \mathrm{MeV}$. This is consistent with the estimate made in ref.[187] based on the heavy mass formalism including the decuplet fields. The effect of the strange quarks is not dramatic. All speculations starting from the first order formula (6.53) should thus be laid at rest. The lesson to be learned here is that many small effects can add up constructively to explain a seemingly large discrepancy like $\Sigma-\sigma_{0} \approx$ $\sigma_{0}$. What is clearly needed are more accurate and reliable low-energy pion-nucleon scattering data to further pin down the uncertainties. For an update on these issues, see Sainio [188].

\subsection{Non-leptonic hyperon decays}

Since the early days of soft-pion techniques, the non-leptonic hyperon decays have been studied using EFT methods (see e.g. the monograph [189]). There are seven such decays, $\Lambda \rightarrow \pi^{0} n, \Lambda \rightarrow \pi^{-} p, \Sigma^{+} \rightarrow \pi^{+} n, \Sigma^{+} \rightarrow \pi^{0} p, \Sigma^{-} \rightarrow \pi^{-} n, \Xi^{0} \rightarrow \pi^{0} \Lambda$ and $\Xi^{-} \rightarrow \pi^{-} \Lambda$. These are compactly written as $H_{a}^{b}$, where $H$ denotes the decaying hyperon, $a=-, 0,+$ gives the charge of the outgoing pion and $b=-, 0,+$ denotes the charge of the decaying hyperon. For example, $\Lambda_{0}^{0}$ stands for $\Lambda \rightarrow \pi^{0} n$. These decays are produced by the strangeness-changing $\Delta S=1$ weak Hamiltonian proportional to $\bar{u} \gamma^{\mu}\left(1+\gamma_{5}\right) s \bar{d} \gamma_{\mu}\left(1+\gamma_{5}\right) u$ (modulo QCD corrections). Under $S U(3) \times S U(3)$ this operator transforms as $\left(8_{L}, 1_{R}\right) \oplus\left(27_{L}, 1_{R}\right)$. Experimentally, the octet piece dominates the decay amplitudes. Therefore, we will neglect the 27 -plet contributions in what follows. The invariant matrix elements take the form

$$
M\left(H_{a}^{b}\right)=G_{F} M_{\pi}^{2} \bar{u}_{B^{\prime}}\left\{A^{(S)}\left(H_{a}^{b}\right)+\gamma_{5} A^{(P)}\left(H_{a}^{b}\right)\right\} u_{B}
$$

where $u_{B}$ denotes the spinor of baryon $B$ and $\bar{u}_{B}=u_{B}^{+} \gamma^{0}$. The $S$-wave decay is parity-violating whereas the $P$-wave decays conserve parity. Isospin symmetry of the strong interactions implies three relations among the amplitudes, separately for the $S-$ and the $P$-waves. These are $M\left(\Lambda_{-}^{0}\right)=-\sqrt{2} M\left(\Lambda_{0}^{0}\right), M\left(\Xi_{-}^{-}\right)=-\sqrt{2} M\left(\Xi_{0}^{0}\right)$ and $\sqrt{2} M\left(\Sigma_{0}^{+}\right)+M\left(\Sigma_{-}^{-}\right)=M\left(\Sigma_{+}^{+}\right)$. Thus, only four amplitudes are independent. $[20,21]$

To leading order in the effective theory, $\Delta S=1$ non-leptonic Hamiltonian reads

$$
H_{\text {eff }}(\Delta S=1)=a \operatorname{Tr}\left(\bar{B}\left\{u^{+} h u, B\right\}\right)+b \operatorname{Tr}\left(\bar{B}\left[u^{+} h u, B\right]\right)+\text { h.c. }
$$

where the explicit expression for the baryon field $B$ is given in appendix $\mathrm{B}$ and the trace runs over the flavor indices. The $S U(3)$ matrix $h$ is a spurion field to project out the octet component, $h_{i}^{j}=\delta_{2}^{j} \delta_{i}^{3}$. From (6.59) it follows for the $S$-waves that $A^{(S)}\left(\Sigma_{+}^{+}\right)=0$, 
$A^{(S)}\left(\Sigma_{-}^{-}\right)=(a-b) / F, A^{(S)}\left(\Lambda_{-}^{0}\right)=-(a+3 b) / \sqrt{6} F$ and $A^{(S)}\left(\Xi_{-}^{-}\right)=(3 b-a) / \sqrt{6} F$. These can be combined to give the Lee-Sugawara relation [190],

$$
A^{(S)}\left(\Lambda_{-}^{0}\right)+2 A^{(S)}\left(\Xi_{-}^{-}\right)+\sqrt{\frac{3}{2}} A^{(S)}\left(\Sigma_{-}^{-}\right)=0
$$

which is experimentally quite well fulfilled (see below). No such simple relation can be derived for the $P$-wave amplitudes since these always involve pole diagrams in which the baryon changes strangeness before or after pion emission. It is worth noting that to leading order, both the $S$ - and the $P$-wave decay amplitudes are independent of the strange quark mass (setting $m_{u}=m_{d}=0$ ). Furthermore, it is not possible to achieve even a descent fit for the $P$-wave amplitudes once the parameters $a$ and $b$ in (6.59) are fixed to reproduce the $S$-waves. This together with a possible solution involving higher dimensional operators is discussed in some detail in refs.[20,21].

Naturally, the question arises what the chiral corrections to these decays are. Bijnens et al. [191] were the first to address this issue. Apart from the standard lowest order chiral meson-baryon Lagrangian (see appendix B) they used for the symmetry breaking the following term:

$\mathcal{L}^{(2)}=a_{1} \operatorname{Tr}\left[\mathcal{M}\left(U+U^{\dagger}\right)\right]+b_{1} \operatorname{Tr}\left[\bar{B}\left(u^{\dagger} \mathcal{M} u^{\dagger}+u \mathcal{M} u\right) B\right]+b_{2} \operatorname{Tr}\left[\bar{B} B\left(u^{\dagger} \mathcal{M} u^{\dagger}+u \mathcal{M} u\right)\right]$

and set $\mathcal{M}=\operatorname{diag}\left(0,0, m_{s}\right)$, i.e. only kaon loops contribute. The parameters $a_{1}, b_{1}$ and $b_{2}$ were determined from the mass spectrum. They worked in the one-loop approximation keeping only the non-analytic terms of the type $m_{s} \ln m_{s} \sim M_{K}^{2} \ln M_{K}^{2}$. No local contact terms were considered. This, of course, introduces a scale dependence, the subtraction point was chosen to be $\mu=1 \mathrm{GeV}$. Tuning the parameters $a$ and $b$ (6.59) to get a best fit to the $S$-wave amplitudes, the following results emerged. The one-loop correction was zero for $A^{(S)}\left(\Sigma_{+}^{+}\right)$, of the order of 30 per cent for $A^{(S)}\left(\Sigma_{-}^{-}\right)$and $A^{(S)}\left(\Lambda_{-}^{0}\right)$ and 75 per cent for $A^{(S)}\left(\Xi_{-}^{-}\right)$. The resulting $S$-wave predictions are no longer satisfactorily agreeing with the data and the Lee-Sugawara relation breaks down. In the $P$-waves, the corrections are even larger, in two cases much bigger than the tree result. None of the $A^{(P)}\left(H_{a}^{b}\right)$ can be reproduced, the discrepancies to the empirical numbers are large. This calculation sheds some doubt on the validity of the chiral expansion in the three flavor sector. Jenkins [192] has recently reinvestigated this topic. Her calculation differs in various ways from the one of ref.[191]. First, she uses the heavy mass formalism and also includes the spin-3/2 decuplet in the effective theory. Second, she accounts for wave-function renormalization and third, includes another higher derivative term. This is nothing but the first term in (4.26) with $\lambda_{6}$ substituted by the spurion $h$. The reason to include this term is the strong $\Delta I=1 / 2$ enhancement. The other assumptions are the same (no counter terms and only the leading non-analytic pieces from the loops are retained. Also, $m_{u}=m_{d}=0$ is assumed). The main result of this calculation is the large cancellation between the octet and decuplet pieces in the loops (already observed 
in the baryon mass calculation [179]), therefore the total loop contribution is considerably reduced. In units of $G_{F} M_{\pi}^{2}$, the results of ref.[192] read: $A^{(S)}\left(\Sigma_{+}^{+}\right)=-0.09(0.06 \pm$ $0.01), A^{(S)}\left(\Sigma_{0}^{+}\right)=-1.41(-1.43 \pm 0.05), A^{(S)}\left(\Sigma_{-}^{-}\right)=1.90(1.88 \pm 0.01), A^{(S)}\left(\Lambda_{-}^{0}\right)=$ $1.44(1.42 \pm 0.01), A^{(S)}\left(\Lambda_{0}^{0}\right)=-1.02(-1.04 \pm 0.01), A^{(S)}\left(\Xi_{-}^{-}\right)=-2.04(-1.98 \pm 0.01)$ and $A^{(S)}\left(\Xi_{0}^{0}\right)=1.44(1.52 \pm 0.02)$. Good agreement between theory and experiment is found. Furthermore, the correction to the Lee-Sugawara relation is small. In case of the $P$-wave amplitudes, the central parameters do not lead to satisfactory description of the data. Indeed, the $A^{(P)}$ are very sensitive to some of the input parameters. What is more important is the observation that in the case of the $P$-waves, the tree level prediction consists of two terms which tend to cancel to a large extent. This suppression of the $S U(3)$ symmetric amplitudes effectively enhances the loop corrections, the terms of the type $m_{s} \ln m_{s}$ are as important as the tree contributions (also they are nominally suppressed). This means that in the $P$-wave amplitudes one has large $S U(3)$ violation, but not a breakdown of the chiral expansion. To get the same accuracy on these $S U(3)$-violating pieces as on the $S U(3)$-symmetric ones which dominate the $S$-wave amplitudes, one would have to work much harder. Clearly, the last word is not spoken here since none of the calculations performed so far accounts for all terms at one loop order, the leading non-analytic corrections are just one part of the whole story.

Neufeld [193] has recently considered the strangeness-changing radiative decays $\Sigma^{+} \rightarrow p \gamma, \Xi^{-} \rightarrow \Sigma^{-} \gamma, \Sigma^{0} \rightarrow n \gamma, \Lambda \rightarrow n \gamma, \Xi^{0} \rightarrow \Lambda \gamma$ and $\Xi^{0} \rightarrow \Sigma^{0} \gamma$ in the relativistic formalism. The general form of the amplitude $B_{b} \rightarrow B_{a}+\gamma(a, b=1, \ldots, 8)$ takes the form

$$
M_{a b}=\bar{u}_{a} i \sigma^{\mu \nu} \epsilon_{\mu}\left(p_{a}-p_{b}\right)_{\nu}\left(A_{a b}+B_{a b} \gamma_{5}\right) u_{b}
$$

The parity-conserving amplitude $A_{a b}$ can be expressed in terms of the magnetic moments and therefore the corresponding counterterms can be fixed. The situation is different for the counterterms related to the parity-violating amplitude $B_{a b}$. They are not known but restricted by CPS-symmetry [103]. The general structure of these counterterms is discussed in ref.[193]. It is argued that the one-loop contributions together with the lowest order contact terms (the pieces which are left for vanishing momenta) are not sufficient to explain the existing data. However, the imaginary parts are uniquely determined by the loop graphs. In particular, for the decays $\Lambda \rightarrow n \gamma$ and $\Xi^{-} \rightarrow \Sigma^{-} \gamma$ one can give a prediction for the asymmetry parameter $\alpha_{a b}=2 \operatorname{Re}\left(A_{a b} B_{a b}^{*}\right) /\left(\left|A_{a b}^{2}\right|+\left|B_{a b}^{2}\right|\right)$ based on the recent measurements for the pertinent branching ratios. These constraints lead to

$$
-0.8 \leq \alpha_{n \Lambda} \leq 0.7,-0.8 \leq \alpha_{\Sigma^{-} \Xi^{-}} \leq 0.8
$$

One can reduce these ranges to some extent by making some plausible assumptions as discussed in ref.[193]. The main conclusion of that paper is that to test the chiral predictions of the asymmetry parameters (6.63) a measurement is called for. In the heavy mass formalism, Jenkins et al. [193] have performed a calculation of the imaginary parts of $A_{a b}$ and $B_{a b}$. They argue that with the exception of the asymmetry parameter for $\Sigma^{+} \rightarrow p \gamma$, good agreement between theory and the available data is obtained.

\subsection{Nuclear forces and exchange currents}


In this section, I will discuss the recent work of Weinberg and collaborators $[194,195,196]$ on the nature of the nuclear forces and the extension of this to mesonexchange currents by Rho et al.[197,198].

On the semi-phenomenological level, nuclear forces are well understood in terms of meson exchange. There is, of course, the long-range pion component first introduced by Yukawa [199]. The intermediate range-attraction between two nucleons can be understood in terms of correlated two-pion exchange, sometimes parametrized in terms of a fictitious scalar-isoscalar $\sigma$-meson with mass $M_{\sigma} \approx 550 \mathrm{MeV}$. $\omega$-meson exchange gives rise to part of the short-range repulsion and the $\rho$ features prominently in the isovectortensor channel, where it cuts down most of the pion tensor potential. Weinberg [194,195] has recently discussed the constraints from the spontaneously broken chiral symmetry on the nature of the nuclear forces. For that, consider the Lagrangian (6.9) including also the four-nucleon terms $\mathcal{L}_{\bar{\Psi} \Psi \bar{\Psi} \Psi}$. Heavy particles are integrated out, their contribution is hidden in the values of the pertinent low-energy constants. Also, consider only nucleons with momenta smaller than some scale $Q$. This induces a $Q$-dependence of the various coupling constants. Alternatively, one could work in the framework of dimensional regularization discussed so far, but the " $Q$-language" is more familiar to nuclear physicists. Clearly, one must set $Q \ll M_{\rho} \simeq m$. Because of this restriction in the nucleon momenta, it is advantageous to use old-fashioned time-ordered perturbation theory in which nucleon propagators are substituted by energy denominators and the integrations run over the three-momenta. The lowest order Lagrangian mandated by chiral symmetry is (for the power counting, see below)

$$
\begin{aligned}
\mathcal{L}= & \mathcal{L}_{\pi \pi}+\mathcal{L}_{\pi N}+\mathcal{L}_{\bar{N} N} \\
= & -\frac{1}{2 D^{2}}\left(\partial_{\mu} \vec{\pi}\right)^{2}-\frac{M_{\pi}^{2}}{2 D} \vec{\pi}^{2} \\
& \left.-\bar{\Psi}_{N}\left[i \partial_{0}-m-\frac{g_{A}}{D F_{\pi}} \vec{\tau} \cdot(\vec{\sigma} \cdot \vec{\nabla}) \vec{\pi}-\frac{1}{2 D F_{\pi}^{2}} \vec{\tau} \cdot\left(\vec{\pi} \times \partial_{0}\right) \vec{\pi}\right)\right] \Psi_{N} \\
& -\frac{1}{2} C_{S}\left(\bar{\Psi}_{N} \Psi_{N}\right)\left(\bar{\Psi}_{N} \Psi_{N}\right)+\frac{1}{2} C_{T}\left(\bar{\Psi}_{N} \vec{\sigma} \Psi_{N}\right) \cdot\left(\bar{\Psi}_{N} \vec{\sigma} \Psi_{N}\right)+\ldots
\end{aligned}
$$

with $\vec{t}$ the isospin generators and $\Psi_{N}$ denotes the nucleon field. We have used stereographic coordinates on $S^{3} \sim S O(4) / S O(3) \sim S U(2) \times S U(2) / S U(2)$ with $D=$ $1+\vec{\pi}^{2} / 4 F_{\pi}^{2} . C_{S}$ and $C_{T}$ are unknown constants and the ellipsis stands for terms with more derivatives, pion mass insertions or nucleon fields. Consider the scattering of $N$, $N \geq 2$, nucleons. There arises a complication in the power counting due to the existence of shallow nuclear bound states. One should not consider the $S$-matrix but rather the effective potential which is defined via the sum of the connected diagrams without $N$ nucleon intermediate states. This stems from the fact that the energy denominators of states involving solely $N$ nucleons are small $\left(\sim Q^{2} / 2 m\right)$ and these cause the perturbation series to diverge at low energies. For a typical pion exchange ladder diagram, this infrared singularity is discussed in detail in ref.[195]. If, however, at least one pion 
exists in the intermediate state, no such problem arises (for a more general discussion of the $N$ nucleon case, see Weinberg [195]). So we are seeking an expansion in the small parameters $Q / m$ and $M_{\pi} / m$. To avoid large factors from time derivatives giving the nucleon mass term, one performs a field redefinition which allows to express the time derivative in terms of other chirally invariant couplings with redefined coefficients,

$$
\left[i \partial_{0}-\frac{1}{2 D F_{\pi}^{2}} \vec{t} \cdot\left(\vec{\pi} \times \partial_{0} \vec{\pi}\right)\right] \Psi_{N}=\left[m+\frac{g_{A}}{D F_{\pi}} \vec{t} \times(\vec{\sigma} \cdot \vec{\nabla}) \vec{\pi}+\ldots\right] \Psi_{N}
$$

This is similar to the heavy mass formalism of ref.[147]. The power counting goes as follows. Consider a vertex of type $i$ with $d_{i}$ derivatives or pion mass insertions involving $n_{i}$ nucleon fields. A graph with $V_{i}$ vertices of type $i$ and $L$ loops scales with $\nu$ powers of $Q$ or $M_{\pi}$

$$
\nu=2-N+2 L+\sum_{i} V_{i}\left(d_{i}+\frac{n_{i}}{2}-2\right)
$$

which follows after making use of some topological identities. For any interaction which is allowed by chiral symmetry (remember that the pion mass counts as order $Q$ ), the coefficient of $V_{i}$ is non-negative. The lowest order diagrams are tree graphs $(L=0)$ with vertices which have $d_{i}=2$ and $n_{i}=0$ or $d_{i}=1$ and $n_{i}=2$ or $d_{i}=0$ and $n_{i}=4$. This just leads to the effective Lagrangian (6.64). One can derive an interaction Hamiltonian (for details, see refs.[194,195])

$$
\begin{aligned}
H_{i n t} & =H_{i n t}^{\pi \pi}+H_{i n t}^{\pi N}+H_{i n t}^{N N} \\
H_{i n t}^{N N} & =\frac{1}{2} C_{S}\left(\bar{\Psi}_{N} \Psi_{N}\right)\left(\bar{\Psi}_{N} \Psi_{N}\right)+\frac{1}{2} C_{T}\left(\bar{\Psi}_{N} \vec{\sigma} \Psi_{N}\right) \cdot\left(\bar{\Psi}_{N} \vec{\sigma} \Psi_{N}\right)
\end{aligned}
$$

From this Hamiltonian, one can easily derive the lowest-order static potential between two nucleons,

$V_{12}\left(\vec{r}_{1}-\vec{r}_{2}\right)=\left[C_{S}+C_{T} \vec{\sigma}_{1} \cdot \vec{\sigma}_{2}\right] \delta\left(\vec{r}_{1}-\vec{r}_{2}\right)-\left(\frac{g_{A}}{F_{\pi}}\right)^{2}\left(\vec{t}_{1} \cdot \vec{t}_{2}\right)\left(\vec{\sigma}_{1} \cdot \vec{\nabla}_{1}\right)\left(\vec{\sigma}_{2} \cdot \vec{\nabla}_{2}\right) Y\left(\left|\vec{r}_{1}-\vec{r}_{2}\right|\right)-\left(1^{\prime} \leftrightarrow 2^{\prime}\right)$

with $Y(r)=\exp \left(-M_{\pi} r\right) / 4 \pi r$ the standard Yukawa function. This potential contains the long range one-pion exchange, the pion pair exchange leading to the intermediate range attraction is hidden in the constant $C_{S}$. In fact, to accomodate the small nuclear binding energies, one has to choose $C_{S}$ large and $C_{T}$ small. This is discussed in detail in ref.[195]. The lowest order approximation of the chirally constrained two-nucleon potential can not explain the mysterious intermediate-range attraction and also shows no sign of the repulsive hard core. Ordonez and van Kolck [196] have considered all terms which are suppressed up to and including $(Q / m)^{2}$ with respect to the leading potential (6.64). These include one-loop diagrams and local contact terms with coefficients of relative order $(Q / m)$ and $(Q / m)^{2}$. Again, intermediate states with nucleons only are excluded. The low-energy constants accompanying the new contact terms have not yet 
been determined, so that the quantitative value of the approach can not be assessed at present. However, some interesting qualitative features emerge (for explicit expressions of the higher order corrections to the two-nucleon potential, see ref.[196]). In this framework, the uncorrelated two-pion exchange together with some of the higher order contact terms furnishes the intermediate-range attraction, the conventional correlated two-pion exchange (pion rescattering, intermediate $\Delta$ states) only appears at next-tonext-to-leading order, $(Q / m)^{4}$ (compare the discussion in ref.[96]). Furthermore, the potential is energy-dependent and contains all terms known from phenomenological approaches. The connection to the meson-exchange potentials can be made if one expresses the low-energy constants in terms of meson couplings and form factors. Weinberg [194] has also made a very interesting observation concerning the three-nucleon forces. To lowest order, these fall into two classes, the one which has only two-body interactions and the other one with genuine three-body forces. The latter class involves diagrams with exclusively non-linear pion interactions, i.e. the emission of two pions at one vertex $(\sim N \pi \pi)$. Summing up all diagrams of this type, the net result is zero. Thus, to leading order, the three-nucleon potential is entirely build from two-body forces without additional genuine three-body ones. Such a behaviour has indeed been observed in exact calculations of the three nucleon system in the continuum (deuteron break-up just above threshold [200]). In this reaction, the momenta involved are small and all observables like the analyzing power etc. can nicely be explained by using only realistic two-body potentials and including some charge symmetry breaking. No genuine three-body forces seem to be needed. Beyond leading order, there is a three-nucleon potential $\sim(Q / m)^{3}$ [196]. It involves some higher order pion-nucleon interactions and also some contact operators consisting of six nucleon fields. The quantitative effects of this potential have not yet been worked out.

Meson exchange currents arise naturally in the meson-exchange picture of the nuclear forces. An external electromagnetic or axial probe does not only couple to the nucleons (impulse approximation) but also to the mesons in flight. The existence of these two-body operators has been verified experimentally [201]. More than 10 years ago, the so-called "chiral filter hypothesis" was introduced [202]. It states that the response of a nucleus to a long-wavelength electroweak probe is given solely by the soft-pion exchange terms dictated by chiral symmetry. Stated differently, all the heavier mesons and nucleon excitations are not seen, even up to energies of the order of 1 GeV. Why this holds true at such energies has not yet been explained. Rho [197] has given a simple argument how the "chiral filter" can occur in nuclei. His lowest order analysis follows closely the one of Weinberg [194]. Any matrix-element $M E$ of the effective potential $V$ or of a current $J_{\mu}$ has the form $M E \sim Q^{\nu} F(Q / m)$, as discussed before. In the presence of a slowly varying external electromagnetic field $A_{\mu}$ (or a weak one), the Hamiltonian takes the form

$$
\begin{aligned}
\mathrm{H}_{\mathrm{eff}} & =\mathrm{H}_{\pi \pi}+\mathrm{H}_{\pi N}+\mathrm{H}_{N N}+\mathrm{H}_{\mathrm{ext}} \\
\mathrm{H}_{\mathrm{ext}} & =\frac{e}{D^{2}}\left[\left(\vec{\pi} \times \partial_{\mu} \vec{\pi}\right)_{3}+\frac{i g_{A}}{2 F_{\pi}} \bar{\Psi}_{N} \gamma_{5} \gamma_{\mu}(\vec{\tau} \times \vec{\pi})_{3} \Psi_{N}\right] A_{\mu}+\ldots
\end{aligned}
$$


and this additional term $\mathrm{H}_{\text {ext }}$ modifies the power counting. Since one derivative is replaced by the external current, the tree graphs $(L=0)$ with the lowest power $\nu$ must fulfill

$$
d_{i}+\frac{1}{2} n_{i}+2=-1
$$

which leads to $d_{i}=0$ and $n_{i}=2$ or $d_{i}=1$ and $n_{i}=0$. In contrast to the case of the nuclear forces, to leading order no four-nucleon contact term contributes. This means that there is no short-ranged two-body current (to lowest order), the exchange current is entirely given in terms of the soft-pion component derived from (6.70). This justifies the chiral filter hypothesis at tree level. Park et al.[198] have also investigated one-loop corrections to the axial-charge operator, the first correction to the pertinent soft-pion matrix-element is suppressed by $(Q / m)^{2}$ [195]. The authors of ref.[198] use the heavy mass formalism which simplifies the calculation considerably. It is shown that the loop corrections are small for distances $r \geq 0.6 \mathrm{fm}$, which means that the lowest order argument of Rho [197] is robust to one-loop order. A vital ingredient in the calculation is that zero-range interactions between the nucleons are omitted, such interactions are suppressed in nuclei by the short-range correlations. This is a very nice result. However, what remains mysterious is why the chiral filter hypothesis works up to so high energies - the answer to this lies certainly outside the realm of baryon CHPT.

Finally, Weinberg [203] has recently used these methods to calculate pion-nucleus scattering lengths. In particular, the chiral symmetry result for the $\pi d$ scattering length does agree with the more phenomenologically derived ones previously [154] and is thus in agreement with the data. Here, the strength of the method lies in the fact that the consistent power counting employed puts the phenomenological result on firm grounds and justifies the assumptions going into its derivation.

\section{STRONGLY COUPLED HIGGS SECTOR}

\subsection{Basic ideas}

The standard model of the strong and electroweak interactions is a spectacularly successful theory. However, we do not understand the dynamics underlying the electroweak symmetry breaking of $S U(2)_{L} \times U(1)_{Y} \rightarrow U(1)_{\mathrm{em}}$. What we know is that this mechanism provides the $W$ and $Z$ bosons with their mass and therefore with their longitudinal degrees of freedom. This indicates that the interactions of longitudinally polarized gauge bosons will provide us with information about the mechanism triggering the symmetry breaking. In the minimal standard model (MSM), which has been studied extensively (see e.g. the monograph by Gunion et al.[204]), the symmetry breaking proceeds as follows. The Lagrangian

$$
\mathcal{L}_{\mathrm{MSM}}=\mathcal{L}_{g}-V
$$


contains a part which includes Yang-Mills and gauge couplings, gauge-fixing, ghost and mass terms as well as Yukawa couplings, $\mathcal{L}_{g}$, plus the Higgs potential $V$. The latter can be written in an $O(4)$ notation as

$$
V(\vec{w}, H)=\frac{\lambda}{4}\left[\left(\vec{w}^{2}+H^{2}\right)-v^{2}\right]^{2}
$$

where $\vec{w}=\left(w^{+}, w^{-}, z\right)$ is a triplet of scalars and $H$ is a fourth scalar field. In case of spontaneous symmetry breaking, the vacuum is asymmetric under the $O(4),<\vec{w}>=0$ and $\langle H\rangle=v$. Shifting the Higgs field so that it has no vacuum expectation value (vev), $H \rightarrow H-v$, one can rewrite (7.2) as

$$
V(\vec{w}, H)=\frac{\lambda}{4}\left(\vec{w}^{2}+H^{2}\right)^{2}+\lambda v H\left(\vec{w}^{2}+H^{2}\right)+\lambda v^{2} H^{2}
$$

which shows that there are three massless scalars $M_{\vec{w}}=0$ and the Higgs field has the mass $M_{H}^{2}=2 \lambda v^{2}$. This is the expected pattern since the $O(4)$ is broken down to $O(3)$, i.e. there should be $(12-6) / 2=3$ Goldstone bosons. These are then eaten up by the originally massless $W^{ \pm}$and $Z$ vector bosons, providing them with their mass and longitudinal degrees of freedom since a massless vector field has only two transverse components. Therefore, the dynamics of the longitudinal components $V_{L}$ (from here on, I collectively denote the $W^{\prime} s$ and $Z^{\prime} s$ by $V$ ) is intimately linked to the symmetry breaking sector. The pattern described above is the MSM Higgs mechanism. The conventionally used complex scalar doublet $\Phi$ is given by $\Phi^{T}=\left(-i w^{+},(H+v-i z) / \sqrt{2}\right)$. From muon decay we know the scale,

$$
<H>=v=\left(\sqrt{2} G_{F}\right)^{-1 / 2}=246 \mathrm{GeV}
$$

with $G_{F}=1.166 \cdot 10^{-5} \mathrm{GeV}^{-2}$ the Fermi constant. However, the scalar coupling strength $\lambda$ is undetermined and thus the Higgs mass is a free parameter of the model. If the Higgs boson is light, of the order of $100 \mathrm{GeV}$, the system including also the gauge and matter sector is amenable to perturbative calculations. This is one possibility. If the Higgs is heavy, say $M_{H} \sim 1 \mathrm{TeV}$, the couplings become large and the symmetry breaking sector is strongly coupled $[205,206]$. Naive perturbation theory fails and one has to invoke nonperturbative methods. Alternatively, there might be no Higgs boson whatsoever but instead the symmetry breaking is induced by some strongly coupled theory. An example for this scenario would be the celebrated technicolor [207] which is a scaled up version of QCD with the replacements $F_{\pi} \rightarrow v$ and $N_{C} \rightarrow N_{T C}$ (the numbers of techicolors might not be three). Such theories generally have a rich spectrum of low-lying resonances, in the case of technicolor these are the techni-rho and the pseudo-Goldstone bosons (the ones which do not give mass to the $\left.V^{\prime} s\right)$. For orientation, the techni-rho is expected to have a mass of $m_{\rho, T C}=\sqrt{3 / N_{T C}}\left(v / F_{\pi}\right) m_{\rho}=2.04 \mathrm{TeV}$ for an $S U(3)_{T C}$ theory with two technifermion families (for a general discussion, see ref.[208]). 
In the absence of an understanding of the symmetry breaking sector, one has to resort to symmetry arguments if one indeed deals with a strongly coupled system. This is what I will assume from here on. In that case, the EFT methods become useful. On general grounds, if the underlying dynamics produces resonances, these must couple to the longitudinal vector bosons. Depending on the actual masses and widths of these resonances, they might be seen at the SSC or the LHC. However, it might also happen that they are too heavy to be detected at the hadron colliders. In that case, the analogy to QCD is again useful. Although the $\rho$-meson sits at $770 \mathrm{MeV}$, it leaves its trace in the particular values of some low-energy constants which are tested at much lower energy, say in threshold $\pi \pi$ scattering, $\sqrt{s}=2 M_{\pi} \approx M_{\rho} / 3$. Similarly, one expects an enhancement in $V_{L} V_{L}$ pairs [209] as a signal of the strongly interacting Higgs sector. One parametrizes the symmetry breaking sector by an effective Lagrangian and replaces the Higgs field by an infinite tower of non-renormalizable operators with a priori unknown coefficients [210]. A particular model of the electroweak symmetry breaking will lead to a definite pattern of the coupling constants. Clearly, at sufficiently low energies, $\sqrt{s} \sim 1 \mathrm{TeV}$, only a finite number of terms will contribute since they can be organized in an energy expansion (as it is the case in QCD). For doing that, one has to set scales. In analogy to QCD, one expects the scale of symmetry breaking to be $\Lambda \simeq 4 \pi v \simeq 3$ $\mathrm{TeV}$ and furthermore estimates the effective theory in the one-loop approximation to be useful up to energies of approximately $\sqrt{s} \simeq \Lambda / 2 \simeq 1.5 \mathrm{TeV}$. If there are lower-lying resonances with mass $M_{R}<\Lambda$, this window is reduced to $\sqrt{s}<M_{R}$. However, this can only be considered as a very rough estimate.

Since in QCD the purest reaction to test the symmetry breaking is $\pi \pi$ scattering, in the strongly coupled Higgs sector much work has been focused on the equivalent reaction $V_{L} V_{L} \rightarrow V_{L} V_{L}$. At high energies, one can make use of the so-called equivalence theorem $[211,212]$. It relates the $S$-matrix containing the unphysical Goldstone bosons $\left(w^{+}, w^{-}, z\right)$ to the $S$-matrix containing the physical longitudinal vector bosons. Any matrix-element $M$ can be decomposed as

$$
M\left(V_{L}, \ldots\right)=M(\vec{w}, \ldots)+\mathcal{O}\left(M_{V} / \sqrt{s}\right)
$$

where the ellipsis stands for matter fields, transverse gauge bosons and so on. The true $S$-matrix element containing the longitudinal degrees of freedom $V_{L}$ and any other physical particles can be written in terms of the Goldstone bosons $w$ as if these were real particles. Clearly, the equivalence theorem becomes operative at high energies, $\sqrt{s}>>M_{V}$. It is important to notice that it holds to all orders in the scalar coupling $\lambda$ [212] which is essential to make it useful for a strongly coupled theory. In fact, there are some subtleties related to eq.(7.5). Let me just discuss one of them. While the 1.h.s.of eq.(7.5) is manifestly gauge-independent, the r.h.s. is not. One can, however, show that all gauge-dependent terms are multplied by a factor $M_{V} / \sqrt{s}$ (at least) and are thus suppressed. Further subtleties of the equivalence theorem are discussed in ref.[213].

There is one more essential phenomenological ingredient which has to be discussed before writing down the effective Lagrangian at next-to-leading order. The MSM 
requires only a breaking from $G=S U(2)_{L} \times U(1)_{Y}$ down to $H=U(1)_{\mathrm{em}}$. However, the Higgs potential (7.3) shows all features of a Goldstone realized chiral $S U(2)$, $G=S U(2) \times S U(2) \rightarrow H=S U(2)$, since $O(4) \sim S U(2) \times S U(2)$ and $O(3) \sim S U(2)$. To see that in more detail, define the field $H$ as a scalar and the $\vec{w}$ as pseudoscalars. Then, $V(\vec{w}, H)$ is invariant under parity. One can eliminate the scalars via a field redifinition, which is nothing but choosing the complex field $\Phi$ in the unitary gauge. The resulting effective theory is a non-linear $\sigma$-model with $F=v$. Such a chiral $S U(2)$ is a feature of a large class of experimentally viable models of the electroweak symmetry breaking. It has the property that the $\rho$-parameter, which measures the ratio of neutral to charged current amplitudes [214]

$$
\rho=\frac{M_{W}^{2}}{M_{Z}^{2} \cos ^{2} \theta_{W}}=1+\mathcal{O}(\alpha)
$$

is protected against strong interaction corrections [215] and only subject to small radiative corrections as denoted by the terms of order $\alpha$ (here, $\alpha$ is a genuine symbol for the electroweak gauge couplings)* In nature, $\rho=0.998 \pm 0.0089$ [216], i.e. $\rho=1$ is fulfilled within a few per cent. In the MSM with $G=S U(2)_{L} \times U(1)_{Y}$, eq.(7.6) holds at tree level. However, in that case there is no relation between the third generator proportional to $z$ and the first two proportional to $w^{ \pm}$and thus loop corrections will lead to sizeable deviations from unity. In case of the larger symmetry group $G=S U(2)_{L} \times S U(2)_{C}$, the three Goldstone bosons belong to a triplet and thus (7.6) is protected. This is completely analogous to the case of isospin symmetry in two-flavor QCD which protects the vector charges against strong renormalization [217]. The additional $S U(2)_{C}$ is called the "custodial" symmetry or one speaks of weak isospin. In most of the following discussion, I will assume such a custodial symmetry or a larger group in which it is embedded. So we are now in the position to write down the pertinent effective Lagrangian and calculate various processes.

\subsection{Effective Lagrangian at next-to-leading order}

In this section, I will discuss the effective Lagrangian to order $p^{4}$ assuming the existence of a custodial $S U(2)$ symmetry. The embedding into a larger group $G$ will also be discussed briefly. In contrast to the case of QCD, one has to quantize the external sources since they are part of a gauge theory. I will work in the Landau gauge $(\xi \rightarrow \infty)$ since in that case the pseudoscalar Goldstone bosons remain massless, $M_{w}=M_{V} / \xi$, and they also decouple from the ghost sector. Furthermore, since $\rho$ is not exactly one in nature, one has to allow for some weak isospin breaking. This can come from the quark mass differences in the doublets (here, only the difference $m_{t}-m_{b}$ or an eventual fourth

* Clearly, one has to define $\rho$ differently if one chooses eq.(7.6) to define the weak mixing angle. 
generation is relevant), hypercharge gauge boson loops and so on. To lowest order $p^{2}$, the effective Lagrangian consists of two terms [210,218,219],

$$
\mathcal{L}^{(2)}=\frac{v^{2}}{4} \operatorname{Tr}\left(D^{\mu} U^{\dagger} D_{\mu} U\right)+\Delta \rho \frac{v^{2}}{8} \operatorname{Tr}\left[\tau^{3}\left(U^{\dagger} D_{\mu} U\right)\right]^{2}
$$

where $U=\exp \left(i \tau^{i} w^{i} / v\right)$ collects the Goldstone bosons and the covariant derivative reads

$$
D_{\mu} U=\partial_{\mu} U+\frac{i}{2} g W_{\mu} U-\frac{i}{2} g^{\prime} B_{\mu} U \tau^{3}
$$

with $W_{\mu}=W_{\mu}^{i} \tau^{i}$. The $S U(2)_{L}$ and $U(1)_{Y}$ gauge couplings are denoted by $g$ and $g^{\prime}$, respectively. In the unitary gauge $U=1$, the first term of (7.7) reduces to the standard bilinear gauge boson terms

$$
\mathcal{L}^{(2)}(U=1, \Delta \rho=0)=\frac{v^{2}}{4}\left(\frac{g^{2}}{2} W_{\mu}^{i} W_{\mu}^{i}+\frac{g^{\prime 2}}{2} B_{\mu} B_{\mu}-g g^{\prime} W_{\mu}^{3} B_{\mu}\right)
$$

so that $M_{W}=g v / 2$ leads to $v=246 \mathrm{GeV}$ as stated before. Diagonalization of (7.9) gives the massless photon and the massive $Z$. The second term in (7.7) brings the expected shift of the $Z$ mass so that $\rho \neq 1$. In fact, to leading order in the quark masses, one finds $\Delta \rho \sim\left(m_{t}-m_{b}\right)^{2} / v^{2} \sim m_{t}^{2} / v^{2}$ [220]. Ramirez [221] has calculated $\Delta \rho$ in two particular models, one with a heavy scalar and the other with a techni-rho. While the effects are of opposite sign in the two models, the contribution to $\Delta \rho$ is too small to be detected at present. However, from this discussion it becomes clear that one has to cleanly separate the known physics (here the $m_{t}^{2}$ contribution) from the unknown one related to the mechanism of the symmetry breaking.

At next-to-leading order, there are much more terms. These have been enumerated by Longhitano [218] some ten years ago. Neglecting custodial symmetry breaking, the order $p^{4}$ Lagrangian reads

$$
\begin{aligned}
\mathcal{L}^{(4)} & =\frac{L_{1}}{16 \pi^{2}}\left[\operatorname{Tr}\left(D^{\mu} U^{\dagger} D_{\mu} U\right)\right]^{2}+\frac{L_{2}}{16 \pi^{2}} \operatorname{Tr}\left(D_{\mu} U^{\dagger} D_{\nu} U\right) \operatorname{Tr}\left(D^{\mu} U^{\dagger} D^{\nu} U\right) \\
& -\frac{i g L_{9 L}}{16 \pi^{2}} \operatorname{Tr}\left(W^{\mu \nu} D_{\mu} U D_{\nu} U^{\dagger}\right)-\frac{i g^{\prime} L_{9 R}}{16 \pi^{2}} \operatorname{Tr}\left(B^{\mu \nu} D_{\mu} U^{\dagger} D_{\nu} U\right) \\
& +\frac{i g g^{\prime} L_{10}}{16 \pi^{2}} \operatorname{Tr}\left(U B^{\mu \nu} U^{\dagger} W_{\mu \nu}\right)
\end{aligned}
$$

with $2 B^{\mu \nu}=\left(\partial_{\mu} B_{\nu}-\partial_{\nu} B_{\mu}\right) \tau^{3}$ and $2 W^{\mu \nu}=\partial_{\mu} W_{\nu}-\partial_{\nu} W_{\mu}-i g\left[W_{\mu}, W_{\nu}\right] / 2$. We have used the convention of Falk et al.[222] and pulled out a factor $\left(1 / 16 \pi^{2}\right)$ so that the $L_{i}$ are numbers of order one. In vectorial theories like e.g. $S U(2)_{L} \times S U(2)_{C} \rightarrow S U(2)_{L+C}$, one has $L_{9 R}=L_{9 L}$. Clearly, the terms proportional to $L_{1,2}$ are related to Goldstone boson scattering while the last three involve external electroweak gauge bosons. There 
are also $\mathcal{O}\left(p^{4}\right)$ symmetry breaking terms which can be found in ref.[218]. They will not be discussed in what follows.

Let me briefly consider theories with larger symmetry groups [223] with an embedding of the custodial symmetry. In that case, there are additional massive scalars, the so-called pseudo-Goldstone bosons. In most models, these are relatively light and typically carry color. So one has to enlarge the covariant derivative (7.8) to include the embedding of $S U(3)_{c} \times S U(2)_{L} \times U(1)_{Y}$ into the larger group $G$. Let $S U(3), S U(2)$ and $U(1)$ be generated by $X^{\alpha}=X^{\alpha A} T^{A}, X^{i}=X^{i A} T^{A}$ and $X=X^{A} T^{A}$, respectively. For $G=G \times G$, the pertinent covariant derivative reads

$$
D_{\mu} U=\partial_{\mu} U+\frac{i}{2} g W_{\mu}^{i} X^{i} U-\frac{i}{2} g^{\prime} B_{\mu} U X+\frac{i}{2} g_{s} G_{\mu}^{\alpha}\left[X^{\alpha}, U\right]
$$

with $U \in G, G_{\mu}^{\alpha}$ the $S U(3)$ color gauge field and $g_{s}$ the strong coupling constant. One also has new operators in the effective Lagrangian. First, there is a mass term for the pseudo-Goldstone bosons, $\mathcal{L}_{M}^{(2)}=-\operatorname{Tr}\left(U M U^{\dagger} M^{\dagger}\right)$, with $M$ the mass matrix of the pseudo-Goldstone bosons. At order $p^{4}$, one has the following new operators for an $S U(2 N) \times S U(2 N)$ global symmetry $(N>1)[224]$

$$
\begin{aligned}
\mathcal{L}_{\text {new }}^{(4)} & =\frac{L_{3}}{16 \pi^{2}} \operatorname{Tr}\left(D^{\mu} U D_{\mu} U^{\dagger} D^{\nu} U D_{\nu} U^{\dagger}\right)+\frac{L_{4}}{16 \pi^{2}} \operatorname{Tr}\left(D_{\mu} U D_{\nu} U^{\dagger} D^{\mu} U D^{\nu} U^{\dagger}\right) \\
& -i g_{s} \frac{L_{13}}{16 \pi^{2}} G_{\mu \nu}^{\alpha} \operatorname{Tr}\left[X^{\alpha}\left(D^{\mu} U D^{\nu} U^{\dagger}+D^{\mu} U^{\dagger} D^{\nu} U\right)\right]
\end{aligned}
$$

This extension becomes important in the context of $V_{L}$ pair production via gluon fusion. Notice that Soldate and Sundrum [233] have argued that in such models the scale of symmetry breaking is not $4 \pi v$, but rather $4 \pi v / N$. Let us now apply the effective Lagrangian to various physical processes.

\subsection{Longitudinal vector boson scattering}

In this section, I will be concerned with elastic scattering of longitudinally polarized vector bosons which is the equivalent to $\pi \pi$ scattering in QCD. After some general remarks on the scattering amplitude, I will discuss some physics issues like e.g. how the longitudinally polarized gauge bosons can be produced at hadron colliders like the SSC or the LHC. I will keep the discussion short but provide sufficient references for the reader who wants to go deeper into this subject.

At low energies, $M_{V}<<\sqrt{s}<<4 \pi v$ (or $M_{R}$, where $M_{R}$ denotes the mass of the lowest resonance), one can derive a low-energy theorem for the scattering process $V_{L} V_{L} \rightarrow V_{L} V_{L}$ in complete analogy with Weinberg's prediction for $\pi \pi$ scattering [62]. It is favorable to work in the Landau gauge so that the Goldstone bosons remain massless. Furthermore, one can make use of the equivalence theorem. Therefore, I will frequently interchange the symbols ${ }^{\prime} V_{L}^{\prime}$ and ${ }^{\prime} w^{ \pm}, z^{\prime}$. To lowest order, the scattering amplitude can 
be given uniquely in terms of a single function $A(s, t, u)$ of the pertinent Mandelstam variables (with $s+t+u=0$ ),

$$
\begin{aligned}
& M\left(w^{+} w^{-} \rightarrow z z\right)=A(s, t, u)=\frac{s}{v^{2}} \\
& M\left(w^{+} w^{-} \rightarrow w^{+} w^{-}\right)=-\frac{u}{v^{2}}, M(z z \rightarrow z z)=0
\end{aligned}
$$

which agress with eq.(4.3) for $F_{\pi} \rightarrow v$ and holds for $\rho=1$. The other channels, i.e. elastic $w^{ \pm} z, w^{+} w^{+}$and $w^{-} w^{-}$scattering, follow by crossing. If there is no custodial symmetry or if it is broken $(\rho \neq 1)$, Chanowitz et al. [225] have generalized (7.13),

$$
M\left(w^{+} w^{-} \rightarrow z z\right)=\frac{s}{v^{2}} \frac{1}{\rho}, M\left(w^{+} w^{-} \rightarrow w^{+} w^{-}\right)=-\frac{u}{v^{2}}\left[4-\frac{3}{\rho}\right]
$$

and the third prediction in (7.13) remains unaffected. The empirical information $\rho \approx 1$ tells us that in the low energy region, symmetry requirements uniquely determine the $V_{L} V_{L}$ scattering amplitudes in terms of a single scale, $v$. It is instructive to see how in the MSM this LET emerges. Exchange of a scalar Higgs field gives

$$
A(s, t, u)=-\frac{M_{H}^{2}}{v^{2}} \frac{s}{s-M_{H}^{2}}=\frac{s}{v^{2}}+\frac{s^{2}}{v^{2} M_{H}^{2}}+\mathcal{O}\left(s^{3}\right)
$$

The first term in the expansion for $s<<v^{2}$ agrees with the LET, the second one depends on the Higgs mass, which in this case is the information about the symmetry breaking sector. Therefore, the idea is to measure deviations from the low-energy behaviour $\sim s / v^{2}$ and try to relate these to the models of the dynamics underlying the symmetry breaking.

For the order $p^{4}$ chiral Lagrangian $(7.7,7.10)$ (with $\Delta \rho=0$ ), the $V_{L} V_{L}$ scattering amplitude contains two unknown coefficients related to the low-energy constants $L_{1}$ and $L_{2}$. This is, of course, completely analogous to Lehmann's [65] one-loop analysis of $\pi \pi$ scattering in the chiral limit. At next-to-leading order, the scattering amplitude is

$$
\begin{aligned}
A(s, t, u) & =\frac{s}{v^{2}}+\frac{1}{16 \pi^{2} v^{4}}\left\{8 L_{1}(\mu) s^{2}+4 L_{2}(\mu) t^{2}\right. \\
& \left.-\frac{s^{2}}{2} \ln \left(-\frac{s}{\mu^{2}}\right)-\frac{1}{12}\left(3 t^{2}+u^{2}-s^{2}\right) \ln \left(-\frac{t}{\mu^{2}}\right)+(t \leftrightarrow u)\right\}
\end{aligned}
$$

Notice that there might be additional constant terms in the $p^{4}$ pieces depending on the renormalization scheme one uses (see ref.[219] for a detailed discussion). It is now instructive to look at the various partial waves for given isospin $(I)$ and angular momentum $(J)$. In the $I=0 S$-wave, the contribution $11 L_{1}(\mu)+7 L_{2}(\mu)$ enters whereas the $I=J=1$ amplitude is sensitive to $L_{2}(\mu)-2 L_{1}(\mu)$ (which actually is a scale-independent 
quantity) and the exotic $S$-wave measures $L_{1}(\mu)-2 L_{2}(\mu)$ [226]. The unitarity effects are entirely determined by the scale $v$ and the scale of dimensional regularization, $\mu$. In the MSM, the one loop effects have been calculated in refs.[227]. Apart from the tree level Higgs exchange (7.15) one has corrections of the type $\ln \left(M_{H}^{2}\right)$. To get an idea in the case of strong coupling, let us imagine models which contain nearby resonances that saturate the low-energy constants (as already discussed in section 2). For a heavy scalar-isoscalar Higgs boson, one finds after matching at the scale $M_{H}[13,224]$

$$
\begin{aligned}
& L_{1}(\mu)=\frac{64 \pi^{3}}{3} \frac{\Gamma_{H} v^{4}}{M_{H}^{5}}+\frac{1}{24} \log \left(\frac{M_{H}^{2}}{\mu^{2}}\right) \\
& L_{2}(\mu)=\frac{1}{12} \log \left(\frac{M_{H}^{2}}{\mu^{2}}\right)
\end{aligned}
$$

where $\Gamma_{H}=3 M_{H}^{3} / 32 \pi v^{2}$ is the standard model width for the Higgs. For a Higgs with mass $M_{H}=2 \mathrm{TeV}$, one finds at the scale $\mu=1.5 \mathrm{TeV}, L_{1}(\mu)=0.33$ and $L_{2}(\mu)=0.01$ (these are the renormalized values). For a model with an isovector-vector exchange, one finds at the scale $M_{\rho}[13,224]$

$$
\begin{aligned}
& L_{1}(\mu)=-192 \pi^{3} \frac{\Gamma_{\rho} v^{4}}{M_{\rho}^{5}}+\frac{1}{24} \log \left(\frac{M_{\rho}^{2}}{\mu^{2}}\right) \\
& L_{2}(\mu)=-L_{1}(\mu)+\frac{1}{8} \log \left(\frac{M_{\rho}^{2}}{\mu^{2}}\right)
\end{aligned}
$$

with $\Gamma_{\rho}$ the pertinent width. Scaling up the QCD $\rho$-meson as described before, one finds $L_{1}(\mu)=-0.31$ and $L_{2}(\mu)=0.38$ at $\mu=1.5 \mathrm{TeV}$. However, there are other contributions to the $L_{i}$. The $\mu$-dependence is actually generated by the Goldstone boson loops and then there is also a fermionic contribution, which can be calculated. For example, Dawson and Valencia [228] have considered the effect of a heavy top quark $\left(m_{t} \leq 200 \mathrm{MeV}\right)$ and found it contributes insignificantly. This again underlines the fact that one has to subtract the known physics from the inferred low-energy constants to get a handle on the underlying dynamics. Let us consider the effect of the particular values of the $L_{1,2}$ as given by eqs. $(7.17,7.18)$ in $V_{L} V_{L}$ scattering [226,229,230,231]. In the case of a heavy Higgs, the $I=J=0$ partial wave is enhanced and $T_{1}^{1}$ is suppressed. The effect is opposite in the case of an isovector-vector exchange, one finds suppression in $T_{0}^{0}$ and enhancement in $T_{1}^{1}$. Notice also that the process $z z \rightarrow z z$ is very sensitive to the actual values of $L_{1,2}$ since its tree level amplitude vanishes. The Higgs model would predict a positive amplitude, while technicolor keeps it essentially zero since the techni-rho does not couple to neutral gauge bosons. For more detailed information on these topics, including also a discussion of various unitarization models, I refer to the recent review by Hikasa [232].

The question arises how to access $V V$ scattering at hadron colliders? In most calculations, one assumes factorization of the production of the $V_{L} V_{L}$ pairs, their scattering and their subsequent decay. Let us focus on the first part of this chain. In 
Fig. 15: Mechanism of producing longitudinal vector gauge boson pairs. These are a) quark-antiquark annihilation, b) gluon fusion and c) vector boson fusion. Solid lines denote (anti)quarks, wiggly lines gluons and dashed lines vector bosons.

$p p$ colliders, there are essentially three mechanisms to produce vector boson pairs, see fig.15. The annihilation of light quarks and antiquarks (fig.15a) gives rise to mostly transversely polarized $V^{\prime} s$ and it is therefore an important background. Appropriate cuts have to be chosen to separate it from the longitudinal pairs. However, as it is the case of the $\rho$-resonance in $e^{+} e^{-}$annihilation, the produced longitudinal pairs are mostly in an isospin-one state [230] making this process sensitive to models with techni-rho's or alike. Also, if there are more general couplings with $L_{9 L} \neq L_{9 R}$, this mechanism can be of importance [222]. Second, there is the fusion of two gluons (fig.15b). Only if the gluons couple to a loop of heavy quarks, a sizeable amount of $V_{L} V_{L}$ pairs is produced. This mechanism is sensitive to isospin-zero resonances and thus a prime way of exploiting a heavy Higgs boson. Also, in models with additional pseudo-Goldstone bosons, it is considerably enhanced due to large color factors [223]. Third, there is the so-called vector boson fusion as shown in fig.15c. It is, of course, only relevant if the Higgs sector is strongly interacting [212]. This mechanism gives the cleanest signal if one is able to isolate it. The luminosities of the initial $V V$ pair (transverse or longitudinal) are generally calculated in the "effective $\mathrm{W}$-approximation" [234], in which the $V^{\prime} s$ are 
considered as partons, i.e. as on-shell, physical bosons. Since they are radiated off the (anti)quarks, one also needs the quark distribution functions in the incoming hadrons. For a detailed study of this topic, see e.g. ref.[208].

After the scattering, one has to detect the $V^{\prime} s$ and separate the scattering process from the background. The most detailed study of the reaction $p p \rightarrow V_{L} V_{L} X$ has been performed by Bagger, Dawson and Valencia [223,224,235] and Bagger [236] has given rate estimates for the LHC and the SSC and discussed the various cuts, tags and vetoes which go into the detection of the "gold-plated" decays $W^{ \pm} \rightarrow \ell^{ \pm} \bar{\nu}_{\ell}$ and $Z \rightarrow \ell^{+} \ell^{-}$ (for $\ell=e, \mu$ ) in each of the final states $W^{+} W^{-}, W^{+} Z, W^{+} W^{+}$and $Z Z$. The tagging and veto methods are discussed in detail in refs.[237,238]. One finds that the events are clean, but have a low rate if there are only high mass resonances, say $M_{R} \geq 2 \mathrm{TeV}$. However, even in the case of a standard model Higgs with $M_{H}=1 \mathrm{TeV}$, the event rates are not large. Isospin-zero resonances are most cleanly seen in the $W^{+} W^{-}$and $Z Z$ channels, while the $W^{+} Z$ channel would be dominated by isospin-one resonances. Models without low mass resonances tend to be most visible in the $W^{+} W^{+}$final state. This means that there is some complementarity in the pertinent signals differentiating the various models of the strongly coupled Higgs sector. For typical cuts and the nominal SSC and LHC luminosities and energies (these are $10^{4}$ and $10^{5} \mathrm{pb}^{-1}$ and $\sqrt{s}=40$ and $16 \mathrm{TeV}$ for the SSC and the LHC, respectively) and assuming $m_{t}=140 \mathrm{GeV}$, the event rates are of the order of $\mathcal{O}(10)$ per year and somewhat larger than the expected background for most models. Clearly, only high statistics experiments will be able to unravel the nature of the mechanism triggering the electroweak symmetry breaking in the case of strong coupling [236]. It is also instructive to see to which kind of new physics the various final states are sensitive in terms of the low-energy constants. If the $V_{L} V_{L}$ pairs are created via $\bar{q} q$ annihilation, the $W_{L}^{+} W_{L}^{-}$and $W_{L}^{ \pm} Z_{L}$ final states are senstive to $L_{9 R, L}$ because the anomalous three gauge boson vertex enters here. This was first pointed out by Falk, Luke and Simmons [222] for hadron colliders like the SSC or the LHC. They estimated from the phenomenology of $W Z$ production that the SSC will be sensitive to $-16 \leq L_{9 L} \leq 7$ and the LHC sets the limits $-22 \leq L_{9 L} \leq 12$ from the transverse momentum distribution of the $Z^{\prime} s$ produced in $p p \rightarrow W^{ \pm} Z+X$. The channel $W^{ \pm} \gamma$ gives limits on the sum $\hat{L}=L_{10}+\left(L_{9 L}+L_{9 R}\right) / 2$, but these are only of the order $|\hat{L}| \leq 50$ (60) for the SSC (LHC). A similar study was recently performed by Dobado and Urdiales [239]. The trilinear gauge boson vertex was also considered by Holdom [240] who investigated technicolor effects on the reaction $e^{+} e^{-} \rightarrow W^{+} W^{-}$. In the case of vector boson fusion, one is naturally sensitive to the couplings $L_{1,2}$. Gluon fusion plays a role in the case of colored pseudo-Goldstone bosons as already stated and would enhance the $W_{L}^{+} W_{L}^{-}$and $Z_{L} Z_{L}$ final states considerably. Further discussions of these topics, concerning also $e^{+} e^{-}$machines, can be found in refs.[232,241]. Finally, constraints on the symmetry breaking sector from a Adler-Weisberger type sum rule have been discussed by Weinberg [242] and Pham [243].

\subsection{Electroweak radiative corrections}


In this section, I will be concerned with the use of EFT methods for calculating electroweak radiative corrections. The high-precision measurements of weak-interaction parameters at LEP have spurred much interest in precise calculations of these radiative corrections from both within and beyond the standard model (SM). For the SM, these have already been calculated using conventional techniques [209,244]. For an introduction on these topics, I refer to the lectures by Peskin [245]. In particular, much work has been focused on the so-called "oblique" corrections, that is corrections to the gauge boson propagators (vacuum polarization effects) [246]. There are several reasons why it is advantagous to consider oblique corrections. First, if there are heavy particles which do not directly couple to the light fermions, they can only be detected via their loop contributions to the gauge boson propagators (for the presently operating accelerators). This is due to the fact that in most models of the electroweak symmetry breaking the decoupling theorem [247] is expected to be inoperative. Second, vacuum polarization effects are universal. They do not depend on the specific process under consideration in contrast to e.g. vertex or box corrections. Furthermore, for reactions involving exclusively light external fermions, one can neglect their masses as compared to the $Z$ mass and therefore only has to take into account the transverse $\left(g_{\mu \nu}\right)$ part of the gauge boson propagator. Following Peskin and Takeuchi [248,249], the oblique corrections can be parametrized in terms of three combinations of the gauge boson self-energies and their derivatives,

$$
\begin{aligned}
\alpha S & =4 e^{2}\left[\Pi_{33}^{\prime}(0)-\Pi_{3 Q}^{\prime}(0)\right] \\
\alpha T & =\frac{e^{2}}{s^{2} c^{2} M_{Z}^{2}}\left[\Pi_{11}(0)-\Pi_{33}(0)\right] \\
\alpha U & =4 e^{2}\left[\Pi_{11}^{\prime}(0)-\Pi_{33}^{\prime}(0)\right]
\end{aligned}
$$

with $s=\sin \theta_{W}, c=\cos \theta_{W}$ and the indices '1,2,3, Q' refer to the $W, Z$ and $\gamma$ particles. The quantity $S$ is isospin-symmetric, whereas $T$ (which is nothing but $\Delta \rho / \alpha$ already discussed) and $U$ are isospin-asymmetric. Since $U$ is related to isospin-breaking in the derivatives of the self-energies, it is supposed to be small in models with a custodial symmetry and often neglected. Notice that Peskin and Takeuchi [249] define these parameters as deviations from the SM ones for a given reference point $m_{t}=150 \mathrm{GeV}$ and $M_{H}=1 \mathrm{TeV}$. The relation to the epsilon parameters of Altarelli et al. [250] is $\epsilon_{1}=\alpha T, \epsilon_{2}=-\alpha U / 4 s^{2}$ and $\epsilon_{3}=\alpha S / 4 s^{2}$. The recent analysis of these authors gives $S=0.1 \pm 1.1, T=-0.06 \pm 0.69$ and $U=1.4 \pm 1.3$ using exclusively LEP results. If one furthermore includes low-energy data from atomic parity violation in $C s$ [251], one finds $S=-0.34 \pm 0.68, T=-0.03 \pm 0.48$ and $U=0.78 \pm 0.98$.

The use of EFT methods in the analysis of oblique corrections was pioneered by Golden and Randall [252], Holdom and Terning [253], Holdom [254] and Dobado et al.[255]. The most lucid discussion of these methods can be found in the paper of Georgi [256] to which I will return later. First, let me give an elementary discussion of the physics behind the parameter $S$ and its relation to the chiral Lagrangian. In the basis of the non-physical fields $A_{\mu}^{3}$ and $B_{\mu}$ it is most simple to calculate the one-loop 
diagram since this gives exactly the mixing which defines $S$. The graph is divergent, it can be made finite by a counterterm of the type

$$
L_{10} g g^{\prime} B_{\mu \nu} W_{a}^{\mu \nu} \operatorname{Tr}\left[U^{\dagger} \tau_{3} U \tau_{a}\right]
$$

from which it immediately follows that

$$
S=-16 \pi L_{10}^{r}
$$

Obviously, one has to specify the renormalization prescription to make this equation precise. As already stressed, $S$ contains a part coming from the SM (denoted by $S_{S M}$ ) and eventually a part due to physics from some higher scale $\Lambda \gg M_{Z}$ (denoted by $\left.S_{N P}\right)$. A particular model for the strongly coupled Higgs sector will lead to a certain value of $L_{10}$ and thus of $S_{N P}$ [253,254]. $S_{S M}$ gets contributions from the quarks and also the SM Higgs. For $M_{H}=1 \mathrm{TeV}$, Altarelli et al. [250] give $S_{S M} \simeq 0.65$ whereas Sint finds $S_{S M} \simeq-0.21$ [219]. It is not yet clear what the source of this discrepancy is. In any case, there is not much room left for non-SM physics if one compares to $S_{\text {exp }}=-0.34 \pm 0.68$. As stressed in refs.[248,254], simple technicolor models give rise to a positive contribution to $S_{N P}$, approximately $[249,257]$

$$
S_{N P} \simeq 0.3 \frac{N_{T F}}{2} \frac{N_{T C}}{3}
$$

for a model with $N_{T C}$ technicolors and $N_{T F}$ technifermions. Since the empirical value of $S$ is negative, QCD-like technicolor theories with a large technisector seem to be ruled out. However, it is possible to construct models which lead to negative values of $S$ [256,258]. In any case, a fully consistent technicolor theory is not yet available and therefore the emiprical information on $S, T$ and $U$ should be used to constrain any model of the electroweak symmetry breaking. Finally, notice that $S$ can be written in terms of a Weinberg sum rule $[219,248,257]$ in complete analogy to the QCD case for $L_{10}$ or $\bar{\ell}_{5}[13,14]$.

Let me now return to the paper of Georgi [256]. First, it is pointed out why the EFT formalism is useful in the calculation of radiative corrections. These reasons are clarity, simplicity and, most important, generality. The EFT allows one to pick up the relevant operators and avoids most complications due to scheme dependence, which is the case in the conventional approach. When one integrates out the particles heavier than some scale $\Lambda$, one produces a tower of non-renormalizable operators in the EFT. This integrating out of the heavy particles in general leads to an effective $\underline{\text { action }}$ which is highly non-local. To arrive at the effective Lagrangian, which is local, one has to perform an operator product expansion. At the scale $\Lambda$, one has to match the physics in the two theories. In that way, the low-energy theory keeps the knowledge about the heavy particles no longer present as active degress of freedom. This matching leads to a power dependence on the heavy particle masses. In addition, there is a 
logarithmic dependence on these masses which originates from using the renormalization group to evolve the EFT to some smaller scale $\mu, \mu<\Lambda$. The general problem of matching in effective field theories which are not necessarily separated by large scales has been discussed by Georgi [259]. The proper matching conditions disentangle the short-distance from the long-distance physics. While the former is incorporated in the coefficients of the effective Lagrangian, the latter remains, of course, explicit in the EFT. In ref.[256], a general analysis of electroweak radiative corrections is performed under a few assumptions (custodial symmetry, no extra Goldstone bosons and no interactions which let the light quarks and leptons participate in the mechanism of the symmetry breaking). First, the energy domain $M_{H}>\Lambda>m_{t}$ is considered. The leading terms beyond the ones from the standard model can be organized in powers of the $U(1)$ gauge coupling. At one loop, one has a term like (7.20) contributing to $S$ and an operator from virtual gauge boson exchange contributing to $T$ plus a whole tower of higher order (suppressed) operators. Going down to the regime where $m_{t}>\Lambda>M_{Z}$, there appear additional contributions to $S$ and $T$, the most important one arises when one integrates out the $t$-quark. At this point, it is obligatory to differentiate between the effective action and the effective Lagrangian since the $b$-quark, which forms a doublet with the $t$, is retained in the EFT. As an example, a calculation is performed for large $\alpha T$, since this is the only oblique correction that scales with $m_{t}^{2}$. It is straightforward to derive the non-perturbative relation

$$
\frac{M_{W}^{2}}{M_{Z}^{2}}=\frac{\rho_{\star}}{2}\left(1+\sqrt{1-\frac{4 \pi \alpha_{\star}}{\sqrt{2} G_{F} M_{Z}^{2} \rho_{\star}}}\right)
$$

with $\rho_{\star}=1+\alpha T$ and $\alpha_{\star}=\alpha\left(M_{Z}\right)$. This relation was originally conjectured in ref.[260], but it can be derived in a much simpler fashion using the EFT approach. Further corrections to eq.(7.23) are also discussed in ref.[256]. In any case, I strongly advice the reader to work through that paper.

Finally, let me just point out that the use of EFT methods in the calculation of radiative electroweak corrections is only in its beginning stage and much more work remains to be done.

\section{MISCELLANEOUS OMISSIONS}

Here, I essentially assemble references for some of the topics not covered in the preceeding sections. However, due to the rapid developments in the field I do not even attempt to offer a complete list but rather refer the reader to his/her favorite data base.

- More mesons : One of the central topics of CHPT was, is and will be the dynamics and interactions of the pseudoscalar Goldstone bosons at low and moderate energies. Of particular interest are the reactions without counterterm contributions at nextto-leading order since they can serve as a clean test of the chiral sector of QCD. These are $K_{L}^{0} \rightarrow \pi^{0} \gamma \gamma$ [99], $K_{S} \rightarrow \gamma \gamma$ [262] and $\gamma \gamma \rightarrow \pi^{0} \pi^{0}$ [43] as well as the 
related process leading to the pion electromagnetic polarizabilities [263]. And, of course, there are plentiful pion, kaon and eta semi- and non-leptonic decays which have not yet been measured with a high precision. A thorough discussion of this can be found in the user's guide to the "chiral temple", known to the non-chiral world also as the Frascati $\Phi$-factory DA $\Phi$ NE [265]. For further references, see also the lectures by Ecker [266] and the recent talk by Leutwyler [267].

- Union of heavy quark and chiral symmetries: In section 6.2 , the nucleon was described as a very heavy (static) source surrounded by a cloud of light pions. This is the physical picture underlying the so-called "heavy quark symmetries" of mesons consisting of one very heavy $(c, b)$ quark and one very light $(u, d, s)$ antiquark. In the limit of the heavy mass becoming infinite, this symmetry becomes exact and one can e.g. derive relations between certain properties of the pseudoscalar $D, B$ mesons with their vectorial excitations $D^{\star}, B^{\star}$. For the emission of pions or kaons from these mesons, e.g. $D^{\star+} \rightarrow D^{0} \pi^{+}$or $B \rightarrow D^{\star} \pi l \nu$, one works with an effective Lagrangian which unites the heavy quark and the chiral symmetry of QCD. This a very new and wide field, and some first work has been reported in refs.[268].

- Large $N_{f}$ : QCD becomes much simpler if one considers the case of a large number $\overline{\text { of colors, }}_{C} \rightarrow \infty[26,27,28]$. Similarly, one hopes to gain a deeper understanding of the chiral expansion by considering the artificial limit of a large number of massless (light) flavors. Some pertinent papers are given in ref.[269]. However, after approximately ten years of experience in the context of Skyrme-type models, which are based on a $1 / N_{C}$ expansion, one is inclined to view this development rather sceptically. Also, recent lattice studies seem to indicate a qualitatively different behaviour for theories with many flavors.

- First reading: There exist several introductory lectures concerned with CHPT and its applications. Let me mention here the ones by Donoghue [270], Ecker [266], Gasser [182] and Leutwyler [271]. More specialized lectures are the ones by Truong [272], Jenkins and Manohar [149] and the author [144]. There are also a few books on the market which deal with EFT methods and CHPT. First, there is the classical book by Georgi [21] and more recently, a broader exposition has been given by Donoghue, Golowich and Holstein [273]. Finally, a state of the art summary as of the fall of 1991 is given in the workshop proceedings [274].

\section{Acknowledgements}

I express my thanks to all my collaborators and everybody who taught me about the material discussed. I am grateful to Véronique Bernard for a careful reading of the manuscript. I also wish to thank all members of the Instiute for Theoretical Physics at the University of Berne for creating most stimulating working conditions. 


\section{APPENDIX A: THE CASE OF $S U(2) \times S U(2)$}

In the two-flavor case, it is most convenient to work with real $O(4)$ fields of unit length, e.g. the pions are collected in $U^{A}(x)=\left(U^{0}(x), U^{i}(x)\right)$ with $U^{T} U=1$. The pertinent covariant derivatives are

$$
\begin{aligned}
& \nabla_{\mu} U^{0}=\partial_{\mu} U^{0}+a_{\mu}^{i} U^{i} \\
& \nabla_{\mu} U^{i}=\partial_{\mu} U^{i}+\epsilon^{i k l} v_{\mu}^{k} U^{l}-a_{\mu}^{i} U^{i}
\end{aligned}
$$

disregarding the isoscalar vector and axial currents. Defining further

$$
\chi^{A}=2 \tilde{B}\left(s^{0}, p^{i}\right), \tilde{\chi}^{A}=2 \tilde{B}\left(p^{0},-s^{i}\right),
$$

where the value of $\tilde{B}$ is slightly different from the one of $B$ (see ref.[14]). The next-toleading order chiral Lagrangian reads:

$$
\mathcal{L}_{\text {eff }}=\sum_{i=1}^{7} \ell_{i} P_{i}+\sum_{j=1}^{3} h_{j} \tilde{P}_{j}
$$

with (we do not exhibit the high-energy terms)

$$
\begin{aligned}
& P_{1}=\operatorname{Tr}\left(\nabla^{\mu} U^{T} \nabla_{\mu} U\right)^{2} \\
& P_{2}=\operatorname{Tr}\left(\nabla_{\mu} U^{T} \nabla_{\nu} U\right) \operatorname{Tr}\left(\nabla^{\mu} U^{T} \nabla^{\nu} U\right) \\
& P_{3}=\left[\operatorname{Tr}\left(\chi^{T} U\right)\right]^{2} \\
& P_{4}=\operatorname{Tr}\left(\nabla^{\mu} \chi^{T} \nabla_{\mu} U\right) \\
& P_{5}=\operatorname{Tr}\left(U^{T} F_{\mu \nu} F^{\mu \nu} U\right) \\
& P_{6}=\operatorname{Tr}\left(\nabla^{\mu} U^{T} F_{\mu \nu} \nabla^{\nu} U\right) \\
& P_{7}=\left[\operatorname{Tr}\left(\tilde{\chi}^{T} U\right)\right]^{2}
\end{aligned}
$$

One can introduce scale-independent low-energy constants $\bar{\ell}_{i}$ via

$$
\ell_{i}^{r}=\frac{\gamma_{i}}{32 \pi^{2}}\left(\bar{\ell}_{i}+\ln \left(M^{2} / \mu^{2}\right)\right)(i=1, \ldots, 6)
$$

with

$$
\gamma_{1}=\frac{1}{3}, \gamma_{2}=\frac{2}{3}, \gamma_{3}=-\frac{1}{2}, \gamma_{4}=2, \gamma_{5}=-\frac{1}{6}, \gamma_{6}=-\frac{1}{3}
$$

Notice that $\ell_{7}$ is not renormalized. The explicit relation of these low-energy constants to the $L_{1}^{r}, \ldots, L_{10}^{r}$ is spelled out in ref.[14]. 


\section{APPENDIX B: $S U(3)$ MESON-BARYON LAGRANGIAN}

Here, I wish to provide the necessary definitions for the three flavor meson-baryon system. It is most convenient to write the eight meson and baryon fields in terms of $S U(3)$ matrices $M$ and $B$, respectively,

$$
\begin{array}{r}
M=\frac{1}{\sqrt{2}}\left(\begin{array}{ccc}
\frac{1}{\sqrt{2}} \pi^{0}+\frac{1}{\sqrt{6}} \eta & \pi^{+} & K^{+} \\
\pi^{-} & -\frac{1}{\sqrt{2}} \pi^{0}+\frac{1}{\sqrt{6}} \eta & K^{0} \\
K^{-} & \bar{K}^{0} & -\frac{2}{\sqrt{6}} \eta
\end{array}\right) \\
B=\left(\begin{array}{ccc}
\frac{1}{\sqrt{2}} \Sigma^{0}+\frac{1}{\sqrt{6}} \Lambda & \Sigma^{+} & p \\
\Sigma^{-} & -\frac{1}{\sqrt{2}} \Sigma^{0}+\frac{1}{\sqrt{6}} \Lambda & n \\
\Xi^{-} & \Xi^{0} & -\frac{2}{\sqrt{6}} \Lambda
\end{array}\right)
\end{array}
$$

with

$$
U(M)=u^{2}(M)=\exp \{2 i M / F\}
$$

and the covariant derivative acting on $B$ reads

$$
\begin{aligned}
D_{\mu} B & =\partial_{\mu} B+\left[\Gamma_{\mu}, B\right] \\
\Gamma_{\mu} & =\frac{1}{2}\left\{u^{\dagger}\left[\partial_{\mu}-i\left(v_{\mu}+a_{\mu}\right)\right] u+u\left[\partial_{\mu}-i\left(v_{\mu}-a_{\mu}\right)\right] u^{\dagger}\right\}
\end{aligned}
$$

with $v_{\mu}$ and $a_{\mu}$ external vector and axial-vector sources. Under $S U(3)_{L} \times S U(3)_{R}, B$ and $D_{\mu} B$ transform as

$$
B^{\prime}=K B K^{\dagger},\left(D_{\mu} B\right)^{\prime}=K\left(D_{\mu} B\right) K^{\dagger}
$$

It is now straightforward to construct the lowest-order $\mathcal{O}(p)$ meson-baryon Lagrangian,

$$
\mathcal{L}_{\mathrm{MB}}^{(1)}=\operatorname{Tr}\left\{i \bar{B} \gamma^{\mu} D_{\mu} B-\stackrel{\circ}{m} \bar{B} B+\frac{1}{2} D \bar{B} \gamma^{\mu}\left\{u_{\mu}, B\right\}+\frac{1}{2} F \bar{B} \gamma^{\mu} \gamma_{5}\left[u_{\mu}, B\right]\right\}
$$

where $\stackrel{\circ}{m}$ stands for the (average) octet mass in the chiral limit. The trace in (B.5) runs over the flavor indices. Notice that in contrast to the $S U(2)$ case, one has two possibilities of coupling the axial $u_{\mu}$ to baryon bilinears. These are the conventional $F$ and $D$ couplings subject to the constraint $F+D=g_{A}=1.26$. At order $\mathcal{O}\left(p^{2}\right)$ the baryon mass degeneracy is lifted by the terms written in eq.(6.61). However, there are many other terms at this order. If one works in the one-loop approximation, one also needs the terms of order $\mathcal{O}\left(p^{3}\right)$. The complete local effective Lagrangians $\mathcal{L}_{\mathrm{MB}}^{(2)}$ and $\mathcal{L}_{\mathrm{MB}}^{(3)}$ are given by Krause [143]. The extension of this to the heavy mass formalism is straightforward, I refer to the article by Jenkins and Manohar [149] (which, however, contains not all terms of $\mathcal{L}_{\mathrm{MB}}^{(2)}$ and none of $\mathcal{L}_{\mathrm{MB}}^{(3)}$ ). 


\section{REFERENCES}

1. E. Euler, Ann. Phys. (Leipzig) 26 (1936) 398;

E. Euler and W. Heisenberg, Z. Phys. 98 (1936) 714;

S. Schweber, "An introduction to relativistic quantum field theory", Harper and Row, New York, 1964.

2. W. Dittrich and M. Reuter, "Effective Lagrangians in Quantum Electrodynamics", Springer Verlag, Heidelberg, 1985.

3. J. Gasser and H. Leutwyler, Phys. Reports C87 (1982) 77.

4. S. L. Adler and R. F. Dashen, "Current Algebras and applications to particle physics", Benjamin, New York, 1968.

5. R. D. Peccei and J. Sola, Nucl. Phys. B281 (1987) 1;

C. A. Dominguez and J. Sola, Z. Phys. C40 (1988) 63.

6. Y. Nambu and G. Jona-Lasinio, Phys. Rev. 122 (1961) 345; 124 (1961) 246.

7. J. Goldstone, Nuovo Cim. 19 (1961) 154.

8. H. Pagels, Phys. Rep. 16 (1975) 219.

9. R. Dashen and M. Weinstein, Phys. Rev. 183 (1969) 1261.

10. S. Weinberg, Phys. Rev. 166 (1968) 1568.

11. S. Coleman, J. Wess and B. Zumino, Phys. Rev. 177 (1969) 2239;

C. G. Callan, S. Coleman, J. Wess and B. Zumino, Phys. Rev. 177 (1969) 2247.

12. S. Weinberg, Physica 96A (1979) 327.

13. J. Gasser and H. Leutwyler, Ann. Phys. (N.Y.) 158 (1984) 142.

14. J. Gasser and H. Leutwyler, Nucl. Phys. B250 (1985) 465.

15. C. Roiesnel and T. N. Truong, Nucl. Phys. B187 (1981) 293.

16. D. G. Boulware and L. S. Brown, Ann. Phys. (N.Y.) 138 (1982) 392.

17. M. Gell-Mann, CALTECH report CTSL-20, 1961;

S. Okubo, Prog. Theor. Phys. 27 (1962) 949.

18. M. Gell-Mann, R. J. Oakes and B. Renner, Phys. Rev. 175 (1968) 2195.

19. M. A. Shifman, A. I. Vainshtein and V. I. Zahkarov, Nucl. Phys. B147 (1979) 385, 448, 519.

20. A. Manohar and H. Georgi, Nucl. Phys. B234 (1984) 189.

21. H. Georgi, "Weak Interactions and Modern ParticlePhysics", Benjamin/Cummings, Reading, MA, 1984.

22. J. Wess and B. Zumino, Phys. Lett. 37B (1971) 95.

23. E. Witten, Nucl. Phys. B223 (1983) 422.

24. Ulf-G. Meißner, Phys. Rep. 161 (1988) 213.

25. J. Schwinger, Phys. Rev. 93 (1951) 664. 
B. DeWitt, "Dynamical theory of groups and fields", Gordon and Breach, New York, 1965.

R. Seeley, Am. Math. Soc. Proc. Symp. Pure Math. 10 (1967) 288.

26. G. 't Hooft, Nucl. Phys. B72 (1974) 461.

27. E. Witten, Nucl. Phys. B160 (1979) 57.

28. G. Veneziano, Nucl. Phys. B117 (1976) 519.

29. C. Riggenbach, J. F. Donoghue, J. Gasser and B. Holstein, Phys. Rev. D43 (1991) 127.

30. G. J. Gounaris and J. J. Sakurai, Phys. Rev. Lett. 21 (1968) 244.

31. S. R. Amendolia et al., Nucl. Phys. B277 (1986) 168.

32. G. Ecker, J. Gasser, A. Pich and E. de Rafael, Nucl. Phys. B321 (1989) 311.

33. J. F. Donoghue, C. Ramirez and G. Valencia, Phys. Rev. D39 (1989) 1947.

34. T. H. Hansson, M. Prakash and I. Zahed, Nucl. Phys. B335 (1990) 67;

V. Bernard and Ulf-G. Meißner, Phys. Lett. B266 (1991) 403;

C. Schüren, E. Ruiz Arriola and K. Goeke, Nucl. Phys. A547 (1992) 612.

35. J. Balog, Phys. Lett. B149 (1984) 197;

D. Ebert and H. Reinhardt, Nucl. Phys. B271 (1986) 188;

E. Ruiz Arriola, Phys. Lett. B253 (1991) 430;

J. Bijnens, C. Bruno and E. de Rafael, preprint CERN-TH.6521/92, 1992.

36. D. Espriu, E. de Rafael and J. Taron, Nucl. Phys. B345 (1990) 22.

37. B. Holdom, J. Terning and K. Verbeck, Phys. Lett. B245 (1990) 612.

38. M. Voloshin and V. Zakharov, Phys. Rev. Lett. 45 (1980) 688.

39. J. F. Donoghue and H. Leutwyler, Z. Phys. C52 (1991) 343.

40. J. F. Donoghue, J. Gasser and H. Leutwyler, Nucl. Phys. B343 (1990) 341.

41. A. Salam and J. Strathdee, Phys. Rev. 184 (1969) 1760;

R. J. Crewther, Phys. Rev. Lett. 28 (1972) 1421;

M. S. Chanowitz and J. Ellis, Phys. Lett. B40 (1972) 397;

J. Schechter, Phys. Rev. D21 (1980) 3393.

42. J. F. Donoghue and D. Wyler, Phys. Rev. D45 (1992) 892.

43. J. Bijnens and F. Cornet, Nucl. Phys. B296 (1988) 557.

44. S. L. Glashow and S. Weinberg, Phys. Rev. Lett. 20 (1968) 224.

45. R. Dashen, Phys. Rev. 183 (1969) 1245.

46. T. Das, G. Guralnick, V. Mathur, F. Low and J. Young, Phys. Rev. Lett. 18 (1967) 759.

47. S. Weinberg, Trans. N.Y. Acad. Sci. 38 (1977) 185.

48. D. B. Kaplan and A. V. Manohar, Phys. Rev. Lett. 56 (1986) 2004.

49. S. Raby and G. B. West, Phys. Rev. D38 (1988) 3488. 
50. G. t'Hooft, Phys. Rev. D14 (1976) 3432;

R. Jackiw and C. Rebbi, Phys. Rev. Lett. 37 (1976) 172;

C. Callan, R. Dashen and D. Gross, Phys. Lett. B63 (1976) 334.

51. K. Choi, C. W. Kim and W. K. Sze, Phys. Rev. Lett. 61 (1988) 794;

K. Choi and C. W. Kim, Phys. Rev. D40 (1989) 890.

52. H. Leutwyler and M. Roos, Z. Phys. C25 (1984) 91.

53. H. Leutwyler, Nucl. Phys. B337 (1990) 108.

54. J. F. Donoghue, B. R. Holstein and Y. C. R. Lin, Phys. Rev. Lett. 55 (1985) 2766;

F. Gilman and R. Kaufman, Phys. Rev. D36 (1987) 2761;

Riazuddin and Fayyazuddin, Phys. Rev. D37 (1988) 149.

55. J. Gasser, Ann. Phys. (N.Y.) 136 (1981) 62.

56. V. Bernard, R. L. Jaffe and Ulf-G. Meißner, Nucl. Phys. B308 (1988) 753.

57. V. Novikov et al., Nucl. Phys. B165 (1980) 55.

58. J. F. Donoghue, B. R. Holstein and D. Wyler, Phys. Rev. Lett. 69 (1992) 3444.

59. R. Socolow, Phys. Rev. B137 (1965) 1221.

60. J. Gasser and H. Leutwyler, Nucl. Phys. B250 (1985) 539.

61. J. Schechter and A. Subbaraman, Int. J. Mod. Phys. A7 (1992) 7135.

62. S. Weinberg, Phys. Rev. Lett. 17 (1966) 616.

63. J. Gasser and H. Leutwyler, Phys. Lett. 125B (1983) 325.

64. J. Gasser and Ulf-G. Meißner, Phys. Lett. B258 (1991) 219.

65. H. Lehmann, Phys. Lett. B41 (1972) 529; Acta Phys. Austriaca Suppl. 11 (1973) 139.

66. V. Bernard, N. Kaiser and Ulf-G. Meißner, Nucl. Phys. B357 (1991) 129.

67. J. L. Petersen, "The $\pi \pi$ Interaction", CERN yellow report 77-04, 1977.

68. S. M. Roy, Phys. Lett. B36 (1971) 353.

69. J. L. Basdevant, C. D. Froggatt, and J. L. Petersen, Nucl. Phys. B72 (1974) 413;

J. L. Basdevant, P. Chapelle, C.Lopez and M. Sigelle, Nucl. Phys. B98 (1975) 285;

C. D. Froggatt and J. L. Petersen, Nucl. Phys. B129 (1977) 89.

70. J. F. Donoghue, B. R. Holstein and G. Valencia, Int. J. Mod. Phys. A2 (1987) 319.

71. V. Bernard, N. Kaiser and Ulf-G. Meißner, Nucl. Phys. B364 (1991) 283.

72. T.T. Wu and C.N. Yang, Phys. Rev. Lett. 13 (1964) 380.

73. W. Ochs, Max-Planck-Institute preprint MPI-Ph/Ph 91-35, 1991.

74. J. F. Donoghue, C. Ramirez and G. Valencia, Phys. Rev. D38 (1988) 2195.

75. M. D. Scadron and H. F. Jones, Phys. Rev. D10 (1974) 967.

76. H. Szadijan and J. Stern, Nucl. Phys. B94 (1975) 163. 
77. N. H. Fuchs, H. Szadijan and J. Stern, Phys. Lett. B238 (1990) 380.

78. C. A. Dominguez, Riv. Nuovo Cim. 8 (1985) No. 6.

79. N. H. Fuchs, H. Szadijan and J. Stern, Phys. Lett. B269 (1991) 183.

80. L. Rosselet et al., Phys. Rev. D15 (1977) 574.

81. L. L. Nemenov, Yad. Fiz. 41 (1985) 980.

82. J. Uretsky and J. Palfrey, Phys. Rev. 121 (1961) 1798.

83. G. Czapek et al., letter of intent CERN/SPSLC 92-44, 1992.

84. R. J. Crewther, Phys. Lett. B176 (1986) 172.

85. see e.g. K. M. Bitar et al., Nucl. Phys. (Proc. Suppl.) 26 (1992) 259.

86. V. Bernard, N. Kaiser and Ulf-G. Meißner, Phys. Rev. D43 (1991) R3557.

87. R. W. Griffith, Phys. Rev. 176 (1968) 1705.

88. N. O. Johannesson and G. Nilson, Nuovo Cim. 43A (1978) 376.

89. C. B. Lang, Fortschritte der Physik 26 (1978) 509;

90. C. B. Lang and W. Porod, Phys. Rev. D21 (1980) 1295.

91. T. N. Truong, Phys. Rev. Lett. 61 (1988) 2526.

92. A. Dobado, M.J. Herrero and T.N. Truong, Phys. Lett. B235 (1990) 134.

93. J. Gasser and Ulf-G. Meißner, Nucl.Phys. B357 (1991) 90.

94. K.L. Au, D. Morgan and M.R. Pennington, Phys. Rev. D35 (1987) 1633.

95. K. M. Watson, Phys. Rev. 95 (1955) 228.

96. Ulf-G. Meißner, Comm. Nucl. Part. Phys. 20 (1991) 119.

97. A. Dobado and J. R. Pelaez, Universidad Complutense de Madrid preprint FT/UCM/10/92, 1992.

98. A. Dobado and J. R. Pelaez, Universidad Complutense de Madrid preprint FT/UCM/9/92, 1992.

99. G. Ecker, A. Pich and E. de Rafael, Phys. Lett. B189 (1987) 363; Nucl. Phys. B291 (1987) 692; Nucl. Phys. B303 (1988) 665.

100. G. Ecker, A. Pich and E. de Rafael, Phys. Rep. in preparation.

101. A. Pich, B. Guberina and E. de Rafael, Nucl. Phys. B277 (1986) 197.

102. J. Kambor, J. Missimer and D. Wyler, Nucl. Phys. B345 (1990) 17.

103. C. Bernard, T. Draper, A. Soni, H. Politzer and M. Wise, Phys.Rev. D32 (1985) 2343.

104. J. Kambor, J. Missimer and D. Wyler, Phys. Lett. B261 (1991) 496.

105. T. N. Truong, Phys. Lett. B207 (1988) 495.

106. Ulf-G. Meißner, in Proc. Fifth International Workshop on "Perspectives in Nuclear Physics at Intermediate Energies", ed. S. Boffi, C. Gioffi degli Atti and M. Giannini, World Scientific, Singapore, 1991. 
107. J. F. Donoghue, E. Golowich and B. R. Holstein, Phys. Rev. D30 (1984) 587.

108. J. Kambor, J. F. Donoghue, B. R. Holstein, J. Missimer and D. Wyler, Phys. Rev. Lett. 68 (1992) 1818.

109. J. Gasser and H. Leutwyler, Phys. Lett. B184 (1987) 83; ibid 188 (1987) 477.

110. H. Leutwyler, Phys. Lett. B189 (1987) 197.

111. P. Gerber and H. Leutwyler, Nucl. Phys. B321 (1989) 387.

112. J. Gasser and H. Leutwyler, Nucl. Phys. B307 (1988) 763.

113. H. Leutwyler, Nucl. Phys. (Proc. Suppl.) 4 (1988) 248.

114. M. Lüscher, Ann. Phys. (N.Y.) 142 (1982) 359.

115. P. Binétruy and M. K. Gaillard, Phys. Rev. D32 (1985) 931.

116. H. Leutwyler, in "Effective field theories of the standard model", ed. Ulf-G. Meißner, World Scientific, Singapore, 1992.

117. P. Gerber and H. Leutwyler, Bern University preprint, 1992.

118. V. Bernard and Ulf-G. Meißner, Phys. Lett. B227 (1989) 465.

119. J. L. Goity, in "Effective field theories of the standard model", ed. Ulf-G. Meißner, World Scientific, Singapore, 1992.

120. M. Lüscher, Nucl. Phys. B354 (1991) 531.

121. H. Neuberger, Phys. Rev. Lett. 60 (1988) 880; Nucl. Phys. B300 [FS22] (1988) 180.

122. P. Hasenfratz and H. Leutwyler, Nucl. Phys. B343 (1990) 241.

123. A. M. Polyakov, Phys. Lett. B103 (1981) 207.

124. I. Dimitrovic, P. Hasenfratz, J. Nager and F. Niedermayer, Nucl. Phys. B350 (1991) 893.

125. A. Hasenfratz et al., Z. Phys. C46 (1990) 257; Nucl. Phys. B356 (1991) 332.

126. M. Göckeler and H. Leutwyler, Nucl. Phys. B350 (1991) 228.

127. I. Dimitrovic, J. Nager K. Jansen and T. Neuhaus, Phys. Lett. B268 (1991) 408.

128. F. C. Hansen, Nucl. Phys. B345 (1990) 685.

129. F. C. Hansen and H. Leutwyler, Nucl. Phys. B350 (1991) 201.

130. S. R. Sharpe, Phys. Rev. D41 (1991) 3233;

S. R. Sharpe, R. Gupta and G. W. Kilcup, Nucl. Phys. B383 (1992) 309;

131. D. Bernard and M. Golterman, Phys. Rev. D46 (1992) 853.

132. H. Leutwyler and A. V. Smilga, Bern University preprint BUTP-92/10, 1992, Phys. Rev. D, in print.

133. P. Hasenfratz and F. Niedermayer, Phys. Lett. B268 (1990) 231.

134. N. D. Mermin and H. Wagner, Phys. Rev. Lett. 17 (1966) 1133;

S. Coleman, Comm. Math. Phys. 31 (1973) 259. 
135. P. Hasenfratz, M. Maggiore and F. Niedermayer, Phys. Lett. B245 (1990) 522.

136. G. Aeppli et al., Phys. Rev. Lett. 62 (1989) 2052.

137. S. Chakravarty, B. I . Halperin and D. R. Nelson, Phys. Rev. Lett. 60 (1988) 1057; Phys. Rev. B39 (1989) 2344.

138. R. J. Birgenau et al., in Proceedings of ICNS'91, 1991.

139. P. Hasenfratz, Nucl. Phys. (Proc. Suppl.) 26 (1992) 247.

140. U.-J. Wiese and H.-P. Ying, Bern University preprint BUTP-92/50, 1992.

P. Hasenfratz and F. Niedermayer, Bern University preprint BUTP-92/46, 1992.

141. P. Langacker and H. Pagels, Phys. Rev. D8 (1971) 4595.

142. J. Gasser, M.E. Sainio and A. Švarc, Nucl. Phys. B 307 (1988) 779.

143. A. Krause, Helv. Phys. Acta 63 (1990) 3.

144. Ulf-G. Meißner, in "Nucleon structure and nucleon resonances", ed. G.A. Miller, World Scientific, Singapore, 1992; Int. J. Mod. Phys. E1 (1992) 561.

145. M. L. Goldberger and S. B. Treiman, Phys. Rev. 110 (1958) 1178.

146. W.E. Caswell and G.P. Lepage, Phys. Lett. B167 (1986) 437;

M.B. Voloshin and M. Shifman, Sov. J. Nucl. Phys. 45 (1986) 463, 47 (1988) 511;

N. Isgur and M.B. Wise, Phys. Lett. B232 (1989) 113, B237 (1990) 527;

E. Eichten and B. Hill, Phys. Lett. B234 (1990) 511;

H. Georgi, Phys. Lett. B242 (1990) 427.

147. E. Jenkins and A.V. Manohar, Phys. Lett. B255 (1991) 558.

148. E. Jenkins and A.V. Manohar, Phys. Lett. B259 (1991) 353.

149. E. Jenkins and A.V. Manohar, in "Effective field theories of the standard model", ed. Ulf-G. Meißner, World Scientific, Singapore, 1992.

150. J. G. Körner and G. Thompson, Phys. Lett. B264 (1991) 185.

151. T. Mannel, W. Roberts and Z. Ryzak, Nucl. Phys. B368 (1992) 264.

152. V. Bernard, N. Kaiser, J. Kambor and Ulf-G. Meißner, Nucl. Phys. B388 (1992) 315.

153. D.G. Caldi and H. Pagels, Phys. Rev. D10 (1974) 3739.

154. T. Ericson and W. Weise, "Pions and Nuclei", Clarendon Press, Oxford, 1988.

155. K.W. Rose et al., Phys. Lett. B234 (1990) 460;

F.J. Federspiel et al., Phys. Rev. Lett. 67 (1991) 1511;

J. Schmiedmayer et al., Phys. Rev. Lett. 66 (1991) 1015.

156. M. Damashek and F. Gilman, Phys. Rev. D1 (1970) 1319;

V.A. Petrunkin, Sov. J. Nucl. Phys. 12 (1981) 278.

157. V. Bernard, N. Kaiser and Ulf-G. Meißner, Phys. Rev. Lett. 67 (1991) 1515.

158. V. Bernard, N. Kaiser, and Ulf-G. Meißner, Nucl. Phys. B373 (1992) 364.

159. M. N. Butler and M. J. Savage, Phys. Lett. B294 (1992) 369. 
160. V. Bernard, N. Kaiser, J. Kambor and Ulf-G. Meißner, Phys. Rev. D46 (1992) 2756.

161. N. Kaiser, in "Baryons as Skyrme Solitons", ed. G. Holzwarth, World Scientific, Singapore, 1993.

162. V. Bernard, N. Kaiser, and Ulf-G. Meißner, Bern University preprint BUTP-92/51, 1992.

163. I.A. Vainshtein and V.I. Zakharov, Sov. J. Nucl. Phys. 12 (1971) 333; Nucl. Phys. B36 (1972) 589;

P. de Baenst, Nucl. Phys. B24 (1970) 633.

164. E. Mazzucato et al., Phys. Rev. Lett. 57 (1986) 3144;

R. Beck et al., Phys. Rev. Lett. 65 (1990) 1841.

165. D. Drechsel and L. Tiator, J. Phys. G: Nucl. Part. Phys. 18 (1992) 449.

166. V. Bernard, J. Gasser, N. Kaiser and Ulf-G. Meißner, Phys. Lett. B268 (1991) 291.

167. V. Bernard, N. Kaiser and Ulf-G. Meißner, Phys. Lett. B282 (1992) 448.

168. S. Scherer and J. H. Koch, Nucl. Phys. A534 (1991) 461.

169. V. Bernard, N. Kaiser and Ulf-G. Meißner, Nucl. Phys. B383 (1992) 442.

170. M. Schumacher, private communication.

171. V. Bernard, N. Kaiser and Ulf-G. Meißner, "Testing nuclear QCD: $\gamma p \rightarrow \pi^{0} p$ at threshold", to appear in the $\pi N$ Newsletter No. 7 (1992).

172. T. P. Welch et al., Phys. Rev. Lett. 69 (1992) 2761.

173. V. Bernard, N. Kaiser, T.-S. H. Lee and Ulf-G. Meißner, Phys. Rev. Lett. 70 (1993) 387.

174. N.M. Kroll and M.A. Ruderman, Phys. Rev. 93 (1954) 233.

175. V. Bernard, N. Kaiser and Ulf-G. Meißner, Phys. Rev. Lett. 69 (1992) 1877.

176. Y. Nambu and D. Lurié, Phys. Rev. 125 (1962) 1429;

Y. Nambu and E. Shrauner, Phys. Rev. 128 (1962) 862.

177. A. del Guerra et al., Nucl. Phys. B107 (1976) 65;

M.G. Olsson, E.T. Osypowski and E.H. Monsay, Phys. Rev. D17 (1978) 2938.

178. T. Kitagaki et al., Phys. Rev. D28 (1983) 436;

L.A. Ahrens et al., Phys. Rev. D35 (1987) 785;

L.A. Ahrens et al., Phys. Lett. B202 (1988) 284.

179. E. Jenkins, Nucl. Phys. B368 (1992) 190.

180. T.P. Cheng and R. Dashen, Phys. Rev. Lett. 26 (1971) 594.

181. G. Höhler, in Landolt-Börnstein, vol.9 b2, ed. H. Schopper (Springer, Berlin, 1983).

182. J. Gasser, in "Hadrons and Hadronic Matter", eds. D. Vautherin et al., Plenum Press, New York, 1990. 
183. R. Koch, Z. Phys. C15 (1982) 161.

184. J. Gasser, H. Leutwyler and M.E. Sainio, Phys. Lett. 253B (1991) 252.

185. L. S. Brown, W. J. Pardee and R. D. Peccei, Phys. Rev. D4 (1971) 2801.

186. J. Gasser, H. Leutwyler and M.E. Sainio, Phys. Lett. 253B (1991) 260.

187. E. Jenkins and A.V. Manohar, Phys. Lett. B281 (1992) 336.

188. M. E. Sainio, in "Effective field theories of the standard model", ed. Ulf-G. Meißner, World Scientific, Singapore, 1992.

189. R. Marshak, Riazuddin and C.P. Ryan, "Theory of weak interactions in particle physics", Wiley-Interscience, New York, 1969.

190. B. W. Lee, Phys. Rev. Lett. 12 (1964) 83;

H. Sugawara, Prog. Theor. Phys. 31 (1964) 213.

191. E. Jenkins, Nucl. Phys. B375 (1992) 561.

192. J. Bijnens, H. Sonoda and M. B. Wise, Nucl. Phys. B261 (1985) 185.

193. H. Neufeld, University of Vienna preprint UWThPh-1992-43, 1992;

E. Jenkins, M. Luke, A. V. Manohar and M. J. Savage, preprint

CERN-TH.6690/92, 1992.

194. S. Weinberg, Phys. Lett. B251 (1990) 288.

195. S. Weinberg, Nucl. Phys. B363 (1991) 3.

196. C. Ordonez and U. van Kolck, Phys. Lett. B291 (1992) 459.

197. M. Rho, Phys. Rev. Lett. 66 (1991) 1275.

198. T.-S. Park, D.-P. Min and M. Rho, preprint SNUTP 92-82, 1992.

199. H. Yukawa, Proc. Phys. Math. Soc. Japan 17 (1935) 48.

200. H. Witala, W. Glöckle and Th. Cornelius, Few Body Systems 6 (1989) 79; Nucl. Phys. 1496 (1989) 446; Phys. Rev. C39 (1989) 384;

I. Slaus, R. Machleidt, W. Tornow, W. Glöckle and H. Witala, Comments Nucl. Part. Phys. 20 (1991) 85;

W. Glöckle, private communication.

201. B. Frois and J.-F. Mathiot, Comments Part. Nucl. Phys. 18 (1989) 291 (and references therein).

202. K. Kubodera, J. Delorme and M. Rho, Phys. Rev. Lett. 40 (1978) 755

M. Rho and G. E. Brown, Comments Part. Nucl. Phys. 10 (1981) 201.

203. S. Weinberg, Phys. Lett. B295 (1992) 114.

204. J. Gunion, H. Haber, G. Kane and S. Dawson, "The Higgs Hunter's Guide", Addison-Wesley, Menlo Park, 1990.

205. D. Dicus and V. Mathur, Phys. Rev. D7 (1973) 3111.

206. B. W. Lee, C. Quigg and H. B. Thacker, Phys. Rev. D16 (1977) 1519.

207. E. Farhi and L. Susskind, Phys. Reports 74 (1981) 277. 
208. E. Eichten, I. Hinchcliffe, K. Lane and C. Quigg, Rev. Mod. Phys. 56 (1984) 579.

209. M. Veltman, Acta Phys. Pol. B8 (1977) 475.

210. T. Appelquist and C. Bernard, Phys. Rev. D22 (1980) 200.

211. J. M. Cornwall, D. N. Levin and G. Tiktopoulos, Phys. Rev. D10 (1974) 1145.

212. M. S. Chanowitz and M. K. Gaillard, Nucl. Phys. B261 (1985) 379.

213. J. Bagger and C. Schmidt, Phys. Rev. D41 (1990) 264;

H.-J. He, Y.-P. Kuang and X. Li, Phys. Rev. Lett. 69 (1992) 2619.

214. D. A. Roos and M. Veltman, Nucl. Phys. B95 (1975) 135.

215. P. Sikvie, L. Susskind, M. Voloshin and V. Zakharov, Nucl. Phys. B173 (1980) 189.

216. U. Amaldi et al., Phys. Rev. D36 (1987) 1385.

217. M. Ademello and R. Gatto, Phys. Rev. Lett. 13 (1964) 264.

218. A. Longhitano, Nucl. Phys. B188 (1981) 118.

219. S. Sint, Diploma thesis, Universität Hamburg, 1991.

220. M. Veltman, Nucl. Phys. B123 (1977) 89;

M. S. Chanowitz, M. A. Furman and I. Hinchcliffe, Phys. Lett. B78 (1978) 285.

221. C. Ramirez, Phys. Rev. D42 (1990) 1726.

222. A. Falk, M. Luke and E. Simmons, Nucl. Phys. B365 (1991) 523.

223. J. Bagger, S. Dawson and G. Valencia, Phys. Rev. Lett. 67 (1991) 2256.

224. J. Bagger, S. Dawson and G. Valencia, preprint FERMILAB-PUB-92/75-T, to appear in Nucl. Phys. B, 1992.

225. M. Chanowitz, M. Golden and H. Georgi, Phys. Rev. D36 (1987) 1490.

226. J. F. Donoghue and C. Ramirez, Phys. Lett. B234 (1990) 361.

227. S. Dawson and S. Willenbrock, Phys. Rev. D40 (1989) 2280;

M. Veltman and F. J. Yndurain, Nucl. Phys. B325 (1989) 1.

228. S. Dawson and G. Valencia, Nucl. Phys. B348 (1991) 23.

229. A. Dobado and M. J. Herrero, Phys. Lett. B228 (1989) 495;

A. Dobado, M. J. Herrero and T. N. Truong, Phys. Lett. B235 (1990) 129.

230. S. Dawson and G. Valencia, Nucl. Phys. B352 (1991) 27.

231. D. A. Dicus and W. W. Repko, Phys. Lett. B228 (1989) 503.

232. K.-I. Hikasa, KEK preprint KEK-TH-319, 1992.

233. M. Soldate and R. Sundrum, Nucl. Phys. B340 (1990) 1.

234. G. L. Kane, W. W. Repko and W. B. Rolnick Phys. Lett. B148 (1984) 367;

S. Dawson, Nucl. Phys. B249 (1985) 42.

235. J. Bagger, S. Dawson and G. Valencia, Phys. Lett. B292 (1992) 137.

236. J. Bagger, talk given at the DPF meeting, Fermilab, 1992. 
237. R. Cahn, S. Ellis, R. Kleiss and W. Stirling, Phys. Rev. D35 (1987) 1626;

U. Baur and E. Glover, Nucl. Phys. B347 (1990) 12;

V. Barger, K. Cheung, T. Han and D. Zeppenfeld, Phys. Rev. D44 (1991) 2701.

238. V. Barger, K. Cheung, T. Han and R. Phillips, Phys. Rev. D42 (1990) 3052;

D. Dicus, J. Gunion, L. Orr and R. Vega, Nucl. Phys. B377 (1992) 31.

239. A. Dobado and M. Urdiales, Phys. Lett. B292 (1992) 128.

240. B. Holdom, Phys. Lett. B258 (1991) 156.

241. M. Chanowitz, Ann. Rev. Nucl. Part. Sci. 38 (1988) 323.

242. S. Weinberg, Phys. Rev. Lett. 65 (1990) 1177.

243. T. N. Pham, Phys. Lett. B255 (1991) 451.

244. A. Sirlin, Nucl. Phys. B71 (1974) 29; Nucl. Phys. B100 (1975) 291; Phys. Rev. D22 (1980) 971;

M. Veltman, Nucl. Phys. B123 (1977) 89;

W. Marciano and A. Sirlin, Phys. Rev. D22 (1980) 2695;

M. Peskin and R. G. Stuart, SLAC-PUB-3725, 1985.

245. M. Peskin, in "Physics at the $100 \mathrm{GeV}$ mass scale", ed. E. C. Brennan, SLACReport-361, 1989.

246. D. Kennedy and B. W. Lynn, Nucl. Phys. B322 (1989) 1;

M. Kuroda, G. Moultaka and D. Schildknecht, Nucl. Phys. B350 (1991) 25.

247. T. Appelquist and J. Carrazone, Phys. Rev. D11 (1975) 2856.

248. M. Peskin and T. Takeuchi, Phys. Rev. Lett. 65 (1990) 964.

249. M. Peskin and T. Takeuchi, Phys. Rev. D46 (1992) 381.

250. G. Altarelli, R. Barbieri and S. Jadach, Nucl. Phys. B369 (1992) 3.

251. W. Marciano and J. Rosner, Phys. Rev. Lett. 65 (1990) 2963.

252. M. Golden and L. Randall, Nucl. Phys. B361 (1991) 3.

253. B. Holdom and J. Terning, Phys. Lett. B247 (1990) 88.

254. B. Holdom, Phys. Lett. B259 (1991) 329.

255. A. Dobado, D. Espriu and M. J. Herrero, Phys. Lett. B255 (1991) 405.

256. H. Georgi, Nucl. Phys. B363 (1991) 301.

257. C. Roiesnel and T. N. Truong, Phys. Lett. B253 (1991) 439.

258. S. Bertolini and A. Sirlin, Phys. Lett. B257 (1991) 179; Phys. Rev. D22 (1980) 971;

E. Gates and J. Terning, Phys. Rev. Lett. 67 (1991) 1840;

M. Dugan and L. Randall, Phys. Lett. B264 (1981) 154.

259. H. Georgi, Nucl. Phys. B361 (1991) 339.

260. M. Consoli, W. Hollik and F. Jegerlehner, Phys. Lett. B227 (1989) 167. 
261. J. F. Donoghue and B. R. Holstein, Phys. Rev. D46 (1992) 4076.

262. G. D'Ambrosio and D. Espriu, Phys. Lett. B175 (1986) 237;

J. L. Goity, Z. Phys. C34 (1987) 341.

263. B. R. Holstein, Comments Nucl. Part. Phys. 19 (1990) 221.

264. J. Bijnens, A. Bramon and F. Cornet, Phys. Rev. Lett. 61 (1988) 1453;

J. F. Donoghue and D. Wyler, Nucl. Phys. B316 (1989) 289;

T. N. Pham, Phys. Lett. B246 (1990) 175;

D. Issler, Preprint SLAC-PUB-4943 and 5200, 1990;

J. Bijnens, Nucl. Phys. B367 (1991) 709;

for an overview, see J. Bijnens, in "Effective field theories of the standard model", ed. Ulf-G. Meißner, World Scientific, Singapore, 1992.

265. The DAFNE Physics Handbook, eds. L. Maiani, G. Pancheri and N. Paver, INFN Frascati, to appear.

266. G. Ecker, preprint CERN-TH.6600/92 and UWThPh-1992-44, 1992.

267. H. Leutwyler, preprint BUTP/92-42, 1992.

268. C. J. C. Im, preprint SLAC-PUB-5627, 1991;

A. Dobado and J. R. Pelaez, Phys. Lett. B286 (1992) 136.

269. M. Wise, Phys. Rev. D45 (1992) 2188;

G. Burdman and J. F. Donoghue, Phys. Lett. B280 (1992) 287;

T. M. Yan et al., Phys. Rev. D46 (1992) 1148;

J. L. Goity, Phys. Rev. D46 (1992) 3929;

U. Kilian, J. G. Körner and D. Pirjol, Phys. Lett. B288 (1992) 360;

A. F. Falk and M. Luke, Phys. Lett. B292 (1992) 119;

J. F. Amundson et al., preprint CERN-TH.6650/92, 1992.

M. A. Novak, M. Rho and I. Zahed, preprint SUNY-NTG-92/27, 1992.

270. J. F. Donoghue, in "Medium energy antiprotons and the quark-gluon structure of hadrons", eds. R. Landua, J. M. Richard and R. Klapish, Plenum Press, New York, 1992.

271. H. Leutwyler, in "Recent Aspects of Quantum Fields", eds. H. Mitter and M. Gausterer, Springer Verlag, Berlin, 1991.

272. T. N. Truong, in "Medium energy antiprotons and the quark-gluon structure of hadrons", eds. R. Landua, J. M. Richard and R. Klapish, Plenum Press, New York, 1992.

273. J. F. Donoghue, E. Golowich and B. R. Holstein, "Dynamics of the Standard Model", Cambridge Univ. Press, Cambridge, 1992.

274. "Effective field theories of the standard model", ed. Ulf-G. Meißner, World Scientific, Singapore, 1992. 